International Electronic Journal of Algebra

VOLUME 23 (2018) 47-114

DOI: $10.24330 /$ ieja.373645

\title{
THE REPRESENTATION TYPE OF THE CHARACTER RING
}

\author{
Tim Fritzsche \\ Received: 06 November 2016; Revised: 06 September 2017; Accepted: 11 September 2017 \\ Communicated by Burcu Üngör
}

\begin{abstract}
Let $R(G)$ be the character ring of a finite group $G$. We consider the question whether the representation type of $R(G)$ is finite or infinite. We show that if $R(G)$ is representation-finite, then $\exp (G)$ is cube-free and the Sylow subgroups of $G$ are cyclic, elementary-abelian, or nonabelian of order 8. Moreover, we give further necessary as well as some sufficient conditions on the structure of $G$ for the finiteness of the representation type of $R(G)$.
\end{abstract}

Mathematics Subject Classification (2010): 19A22, 20C99

Keywords: Character ring, representation type, almost 2-transitive groups of cube-free order

\section{Introduction}

Let $G$ be a finite group and $R(G)$ be the character ring of $G$, i.e. the Grothendieck ring of the semiring of complex characters of $G$ with canonical addition and multiplication induced by the tensor product of characters. The aim of this article is to determine the representation type of $R(G)$ only by information on the group structure of $G$.

It is not difficult to show $R(G)=\mathbb{Z}[\operatorname{Irr}(G)]$ where $\operatorname{Irr}(G)$ denotes the set of irreducible (complex) characters of $G$. Thus, $R(G)$ is a $\mathbb{Z}$-order in the $\mathbb{Q}$-algebra $\mathbb{Q} \otimes_{\mathbb{Z}} R(G)$. That is why we can apply the well-developed representation theory of orders (see e.g. [12]). This approach has been pursued successfully for the determination of the representation type of the group ring $\mathbb{Z} G$ of $G[3,23,24,31]$ and the Burnside ring of $G$ [35]. Since $\mathbb{Z} G$ and $R(G)$ are isomorphic for an abelian group $G$, we obtain that $R(G)$ is representation-finite if and only if all Sylow subgroups of $G$ are cyclic and the exponent $\exp (G)$ of $G$ is cube-free.

For a nonabelian group $G$, the structure of $R(G)$ differs from the structure of $\mathbb{Z} G$. However, we have strong criteria for the finiteness of the representation type of a commutative order. Jones has established a local-global principle [31] and Jacobinski as well as Drozd and Rolter proved that for a Dedekind ring $R$ with quotient field $\mathbb{K}$, the representation type of a separable commutative $R$-order $\Lambda$ can be read off the structure of the $\Lambda$-module $\Lambda^{\prime} / \Lambda$ where $\Lambda^{\prime}$ denotes the maximal $R$-order in $\mathbb{K} \otimes_{R} \Lambda[16,30]$ (see also [22] for the proof of this result). 
We will see how these criteria can be applied to determine the representation type of $R(G)$ in Section 2. There, we recall some properties of the maximal order $R(G)^{\prime}$ and construct special functions in $R(G)^{\prime}$. This leads to a reduction of our problem to a question on the rational $p^{\prime}$-sections of $G$. In particular, we obtain that $R(G)$ has infinite representation type if $G$ contains an element of order $p^{3}$ for some prime $p$.

In Section 3, we show that if $R(G)$ is representation-finite, then $G$ has at most one nonabelian composition factor and we determine all possible isomorphism types a component of $G$ may have. Afterwards, we prove that if $R(G)$ has finite representation type, then the Sylow $p$-subgroups of $G$ are cyclic of order at most $p^{2}$, elementary-abelian, or nonabelian of order 8 . We follow the same approach as Costantini and Jabara did in [10] where groups in which any two cyclic subgroups of the same order are conjugate are considered. Here, the classification of finite simple groups is used several times.

In Section 4, we try to find sufficient conditions for $R(G)$ having finite representation type. At first, we show that if $G$ has only cyclic Sylow subgroups, then $R(G)$ is representation-finite if and only if $|G|$ is cube-free. Next, we consider groups with elementary-abelian Sylow $p$-subgroups. We will not get complete results there, but these results will be sufficient to prove that for a group $G$ of odd order, $R(G)$ has finite representation type if and only if all Sylow subgroups of $G$ are cyclic of order $\leq p^{2}$. We close by giving a list of properties which a group of even order whose character ring is representation-finite must have.

\section{Reduction on rational $p^{\prime}$-sections}

At first, we fix some notation used in this paper. Throughout this article, $G$ is a finite group with character ring $R(G)$. For $g, h \in G$, we write $g \sim_{G} h$ or simply $g \sim h$ if $g$ and $h$ are conjugate in $G$. The conjugacy class of $g$ is $\operatorname{cl}_{G}(g)$ and $C_{G}(g)$ denotes the centralizer of $g$ in $G$. For a subgroup $H \leq G, N_{G}(H)$ is the normalizer and $C_{G}(H)$ the centralizer of $H$ in $G$.

Recall that for a prime $p$ and some $g \in G$, there exist unique elements $x, y \in G$ such that $g=x y=y x$, the order of $x$ is a power of $p$, and the order of $y$ is prime to $p$. Then, $x$ is called the $p$-part of $g$ and $y$ is the $p^{\prime}$-part of $g$. We will write $g_{p}$ for the $p$-part and $g_{p^{\prime}}$ for the $p^{\prime}$-part of $g$ in the following. Moreover, we can define an equivalence relation on $G$ where $g, h \in G$ are equivalent if and only if $g_{p}$ and $h_{p}$ are conjugate in $G$. The equivalence classes are called $p$-sections of $G$. Analogously, we obtain an equivalence relation on $G$ if we replace $g_{p}$ by $g_{p^{\prime}}$ and $h_{p}$ by $h_{p^{\prime}}$ respectively. Here, the equivalence classes are called $p^{\prime}$-sections of $G$. 
The notations in connection with characters coincide with the notations in [29]. Additionally, we write $\mathbb{1}$ for the trivial character of a finite group and set $\zeta_{n}=e^{2 \pi i / n}$ for $n \in \mathbb{N}$. If $\mathbb{K}$ is an algebraic number field, $\mathcal{O}_{\mathbb{K}}$ denotes the ring of integers of $\mathbb{K}$. Moreover, for a finite field extension $\mathbb{L} / \mathbb{K}$ and $x \in \mathbb{L}$, we write $\mathrm{N}_{\mathbb{L} / \mathbb{K}}(x)$ for the norm of $x$ and $\operatorname{Tr}_{\mathbb{L} / \mathbb{K}}(x)$ or just $\operatorname{Tr}(x)$ for the trace of $x$. Finally, if $R$ is a Dedekind ring with quotient field $\mathbb{K}$ and $\Lambda$ is an $R$-order in a separable commutative $\mathbb{K}$-algebra $A$, then $\Lambda^{\prime}$ denotes the maximal $R$-order in $A$. For a prime ideal $\mathfrak{p}$ of $R$, we set $\Lambda_{\mathfrak{p}}=R_{\mathfrak{p}} \otimes_{R} \Lambda$ for the localization at $\mathfrak{p}$. We say that $\Lambda$ is representation-finite if there exist only finitely many isomorphism classes of indecomposable $\Lambda$-lattices. Recall that a $\Lambda$-lattice is a finitely generated $\Lambda$-module which is projective as an $R$-module.

As mentioned in the introduction, we will make use of some strong general results on the representation type of orders, for example of the following.

Theorem 2.1 (Jacobinski/Drozd-Router). Let $R$ be a Dedekind domain whose quotient field $\mathbb{K}$ is a global field or the completion of a global field. Let $A$ be a separable commutative finite-dimensional $\mathbb{K}$-algebra, and $\Lambda$ be an $R$-order in $A$. The order $\Lambda$ is representation-finite if and only if

(1) for the $\Lambda$-module $\Lambda^{\prime} / \Lambda$, there exists a generating set consisting of two elements,

(2) the $\Lambda$-module $\operatorname{rad}\left(\Lambda^{\prime} / \Lambda\right)$ is cyclic.

However, in general, it will not suffice to set $\Lambda=R(G)$ and apply the theorem since it might be too complicated to find a minimal generating system for the $R(G)$ module $R(G)^{\prime} / R(G)$ or $\operatorname{rad}\left(R(G)^{\prime} / R(G)\right)$ respectively. A more promising way is to combine Theorem 2.1 with the local-global principle of Jones [31]. This leads to the following result [36].

Corollary 2.2 (Reichenbach). Let $R$ be a Dedekind domain whose quotient field $\mathbb{K}$ is a global field or the completion of a global field. Let $A$ be a separable commutative finite-dimensional $\mathbb{K}$-algebra, and $\Lambda$ be an $R$-order in $A$. Moreover, let $a \in R$ such that $a \Lambda^{\prime} \subseteq \Lambda$ for the maximal $R$-order $\Lambda^{\prime}$ in $A$. Then, $\Lambda$ has finite representation type if and only if for any prime ideal $\mathfrak{p}$ of $R$ containing aR, the $R_{\mathfrak{p}}$-order $\Lambda_{\mathfrak{p}}$ is representation-finite.

In this article, we are going to determine the representation type of $R(G)$ by applying Corollary 2.2 with $R=\mathbb{Z}, \mathbb{K}=\mathbb{Q}$, and $\Lambda=R(G)$. Hence, for certain primes $p$, we have to determine the respective representation type of the $\mathbb{Z}_{(p)}$-order $R(G)_{p}$. This can be done by applying Theorem 2.1 with $R=\mathbb{Z}_{(p)}, \mathbb{K}=\mathbb{Q}$, and $\Lambda=R(G)_{p}$. 
Most of the assumptions of Theorem 2.1 and Corollary 2.2 are clearly satisfied in these cases. It remains to show that $\mathbb{Q} \otimes R(G)$ (we always write $\otimes$ for $\otimes_{\mathbb{Z}}$ in this article) as well as $\mathbb{Q} \otimes_{\mathbb{Z}_{(p)}} R(G)_{p}$ are separable algebras, and that there exists a positive integer $a$ such that $a R(G)^{\prime} \subseteq R(G)$. The first assertion follows from the fact that $\mathbb{Q}\left(\zeta_{|G|}\right) \otimes R(G)$ is a split semisimple $\mathbb{Q}\left(\zeta_{|G|}\right)$-algebra. The second assertion holds due to [20] where $|G| \cdot R(G)^{\prime} \subseteq R(G)$ has been shown. This also means that we only have to consider the prime divisors of $|G|$ in the following.

As we have already seen, the maximal order $R(G)^{\prime}$ plays an important role in our approach. That is why we recall some facts about $R(G)^{\prime}$. The general theory of orders yields that $R(G)^{\prime}$ consists of those functions of $\mathbb{Q} \otimes R(G)$ whose values on the elements of $G$ are algebraic integers. This leads to a further description which gives more information on the structure of $R(G)^{\prime}$. Here, we make use of the notion of $\mathbb{Q}$-classes of $G$.

For $g \in G$, the $\mathbb{Q}$-class of $g$ is the set of all elements of $G$ which are $\mathbb{Q}$-conjugate to $g$. Let $n:=\exp (G)$. We say that the elements $g, h \in G$ are $\mathbb{Q}$-conjugate in $G$ if an integer $z$ with $\operatorname{gcd}(z, n)=1$ and some $\sigma \in \operatorname{Gal}\left(\mathbb{Q}\left(\zeta_{n}\right) / \mathbb{Q}\right)$ with $\sigma\left(\zeta_{n}\right)=\zeta_{n}^{z}$ exist such that $g$ and $h^{z}$ are conjugate in $G$. This definition is due to Berman who has introduced the more general notion of $\mathbb{K}$-classes for a field $\mathbb{K}$ whose characteristic is either 0 or prime to $|G|[1,2]$. In the case $\mathbb{K}=\mathbb{Q}$ this yields the $\mathbb{Q}$-classes of $G$ as defined above.

We remark that in general, a $\mathbb{Q}$-class of $G$ is not a rational conjugacy class of $G$. A conjugacy class $C$ of $G$ is a rational conjugacy class if and only if $C$ is already a $\mathbb{Q}$-class. It can easily be seen that two elements $g, h \in G$ are $\mathbb{Q}$-conjugate if and only if the cyclic subgroups $\langle g\rangle$ and $\langle h\rangle$ are conjugate.

As for the conjugacy classes of $G$, we can define $p$-sections and $p^{\prime}$-sections for the $\mathbb{Q}$-classes of $G$. The group $\operatorname{Gal}\left(\mathbb{Q}\left(\zeta_{n}\right) / \mathbb{Q}\right)$ acts on the conjugacy classes of $G$ by $\sigma\left(\operatorname{cl}_{G}(x)\right)=\operatorname{cl}_{G}\left(x^{t}\right)$ for $\sigma \in \operatorname{Gal}\left(\mathbb{Q}\left(\zeta_{n}\right) / \mathbb{Q}\right), x \in G$, and the well defined integer $t$ satisfying $1 \leq t \leq n$ as well as $\sigma\left(\zeta_{n}\right)=\zeta_{n}^{t}$. If $\mathcal{S}$ is a union of conjugacy classes $C_{1}, \ldots, C_{k}$, we set $\sigma(\mathcal{S})=\sigma\left(C_{1}\right) \cup \ldots \cup \sigma\left(C_{k}\right)$ accordingly. Thus, $\operatorname{Gal}\left(\mathbb{Q}\left(\zeta_{n}\right) / \mathbb{Q}\right)$ also acts on the $p$-sections and the $p^{\prime}$-sections of $G$ respectively. We are interested in the $p^{\prime}$-sections primarily.

Definition 2.3. Let $p$ be a prime, $\mathcal{S}$ be a $p^{\prime}$-section of $G$, and $\mathcal{G}:=\operatorname{Gal}\left(\mathbb{Q}\left(\zeta_{\exp (G)}\right) / \mathbb{Q}\right)$. Then, we call, $\bigcup_{\sigma \in \mathcal{G}} \sigma(\mathcal{S})$ a rational $p^{\prime}$-section of $G$.

This means that a rational $p^{\prime}$-section of $G$ is a $p^{\prime}$-section of $\mathbb{Q}$-classes. Two elements $g, h \in G$ lie in the same rational $p^{\prime}$-section if and only if $\left\langle g_{p^{\prime}}\right\rangle$ and $\left\langle h_{p^{\prime}}\right\rangle$ are conjugate in $G$. In particular, a rational $p^{\prime}$-section can contain nonrational conjugacy classes. 
We assign algebraic number fields to the conjugacy classes and the $\mathbb{Q}$-classes of $G$ as follows. Let $\mathcal{C}_{1}, \ldots, \mathcal{C}_{k}$ be the $\mathbb{Q}$-classes of $G$ and let $C_{1}, \ldots, C_{\ell}$ be the conjugacy classes of $G$ such that $C_{i} \subseteq \mathcal{C}_{i}$ for $i=1, \ldots, k$. For $x_{i} \in C_{i}$, we set $\mathbb{Q}\left(C_{i}\right)=$ $\mathbb{Q}\left(\chi\left(x_{i}\right): \chi \in \operatorname{Irr}(G)\right)$. Obviously, this definition is independent of the choice of $x_{i}$. Moreover, we get $\mathbb{Q}\left(\mathcal{C}_{i}\right)=\mathbb{Q}\left(C_{i}\right)$ if we set $\mathbb{Q}\left(\mathcal{C}_{i}\right)=\mathbb{Q}\left(\chi\left(y_{i}\right): \chi \in \operatorname{Irr}(G)\right)$ for some $y_{i} \in \mathcal{C}_{i}$. These definitions give rise to a $\mathbb{Q}$-algebra $\bigoplus_{i=1}^{k} \mathbb{Q}\left(\mathcal{C}_{i}\right)$ where the product of two elements is given by component-wise multiplication. By using this, we obtain an alternative description for $R(G)^{\prime}[20]$.

Theorem 2.4. Let $\mathcal{C}_{1}, \ldots, \mathcal{C}_{k}$ be the $\mathbb{Q}$-classes of $G$, and $\left\{x_{1}, \ldots, x_{k}\right\}$ be a set of representatives of these $\mathbb{Q}$-classes with $x_{i} \in \mathcal{C}_{i}$ for $i=1, \ldots, k$. Then, we have an isomorphism of $\mathbb{Q}$-algebras

$$
\mathbb{Q} \otimes R(G) \rightarrow \bigoplus_{i=1}^{k} \mathbb{Q}\left(\mathcal{C}_{i}\right), \quad a \otimes \psi \mapsto\left(a \psi\left(x_{1}\right), \ldots, a \psi\left(x_{k}\right)\right) .
$$

This yields an isomorphism

$$
R(G)^{\prime} \cong \bigoplus_{i=1}^{k} \mathcal{O}_{\mathbb{Q}\left(\mathcal{C}_{i}\right)}
$$

Remark 2.5. The determination of the degree of the field extension $\mathbb{Q}\left(\mathcal{C}_{i}\right) / \mathbb{Q}$ is a crucial part in the proof of Theorem 2.4. This uses the fact that given a conjugacy class $C$ of $G$ and some $x \in C$, there is a canonical isomorphism between $\operatorname{Gal}\left(\mathbb{Q}\left(\zeta_{|\langle x\rangle|}\right) / \mathbb{Q}(C)\right)$ and $N_{G}(\langle x\rangle) / C_{G}(x)$ (see e.g. [34]).

Using the description $\bigoplus_{i=1}^{k} \mathcal{O}_{\mathbb{Q}\left(\mathcal{C}_{i}\right)}$ instead of $R(G)^{\prime}$ has a significant advantage. While for $\varphi, \eta \in R(G)^{\prime}$, it is difficult to decompose $\varphi \eta$ into a linear combination of the irreducible characters of $G$, the product of $a, b \in \bigoplus_{i=1}^{k} \mathcal{O}_{\mathbb{Q}\left(\mathcal{C}_{i}\right)}$ with $a=$ $\left(a_{1}, \ldots, a_{k}\right)$ and $b=\left(b_{1}, \ldots, b_{k}\right)$ is just $a b=\left(a_{1} b_{1}, \ldots, a_{k} b_{k}\right)$. That is why we identify the class functions in $R(G)^{\prime}$ with elements of $\bigoplus_{i=1}^{k} \mathcal{O}_{\mathbb{Q}\left(\mathcal{C}_{i}\right)}$ and the class functions in $\mathbb{Q} \otimes R(G)$ with elements of the algebra $\bigoplus_{i=1}^{k} \mathbb{Q}\left(\mathcal{C}_{i}\right)$ in the following.

This leads us to the question which elements of $\mathbb{Z}_{(p)} \otimes \bigoplus_{i=1}^{k} \mathcal{O}_{\mathbb{Q}\left(\mathcal{C}_{i}\right)}$ also lie in $R(G)_{p}$ if $p$ is a prime dividing $|G|$. We are going to answer this question for some special integer-valued class functions which have nonzero values on only a few $\mathbb{Q}$ classes in Lemmas 2.6 and 2.7. Prior to this we need to introduce further notation. Let $\mathcal{S}$ be a union of $\mathbb{Q}$-classes of $G$. For a ring $T$ of algebraic numbers, we define

$$
\mathrm{Ch}_{T}(\mathcal{S}):=\left\{\psi=\sum_{\chi \in \operatorname{Irr}(G)} a_{\chi} \chi: a_{\chi} \in T, \psi(z)=0 \text { for } z \notin \mathcal{S}\right\} .
$$

Moreover, we set $\mathrm{Ch}(\mathcal{S}):=\mathrm{Ch}_{\mathbb{Z}}(\mathcal{S})$. 
Lemma 2.6. Let $p$ be a prime and $\mathcal{S}:=\left\{x \in G: p \nmid\left|C_{G}(x)\right|\right\}$. Then,

$$
R(G)_{p}^{\prime} \cap \mathrm{Ch}_{\mathbb{Q}}(\mathcal{S})=\mathrm{Ch}_{\mathbb{Z}_{(p)}}(\mathcal{S})
$$

Proof. Let $\varepsilon=\zeta_{\exp (G)}$, and $\left\{z_{1}, \ldots, z_{k}\right\}$ be a transversal for the conjugacy classes contained in $\mathcal{S}$. It suffices to show that any $\varphi_{1} \in \operatorname{Ch}(\mathcal{S})$ satisfying $\varphi_{1}\left(z_{i}\right) \in p \mathbb{Z}[\varepsilon]$, $i=1, \ldots, k$, has the form $\varphi_{1}=p \varphi_{2}$ for some $\varphi_{2} \in \operatorname{Ch}(\mathcal{S})$. Suppose $\varphi_{1}\left(z_{i}\right)=p r_{i}$ for some $r_{i} \in \mathbb{Z}[\varepsilon], i=1, \ldots, k$. For $g \in G$, let $\vartheta_{g}$ be the function with $\vartheta_{g}(x)=1$ if $x \in G$ is conjugate to $g$ and $\vartheta_{g}(x)=0$ else. Then, the orthogonality relations yield

$$
\vartheta_{g}=\frac{1}{\left|C_{G}(g)\right|} \sum_{\chi \in \operatorname{Irr}(\mathrm{G})} \chi\left(g^{-1}\right) \chi
$$

That is why

$$
\varphi_{1}=\sum_{i=1}^{k} \operatorname{pr}_{i} \vartheta_{z_{i}}=p \sum_{\chi \in \operatorname{Irr}(G)}\left(\sum_{i=1}^{k} \frac{r_{i}}{\left|C_{G}\left(z_{i}\right)\right|} \chi\left(z_{i}^{-1}\right)\right) \chi .
$$

The denominators of the inner sums are coprime to $p$ by assumption. Applying this and $\varphi_{1} \in \mathrm{Ch}(\mathcal{S})$, we conclude that the inner sums are contained in $\mathbb{Z}$ respectively. Hence,

$$
\varphi_{2}:=\sum_{\chi \in \operatorname{Irr}(G)}\left(\sum_{i=1}^{k} \frac{r_{i}}{\left|C_{G}\left(z_{i}\right)\right|} \chi\left(z_{i}^{-1}\right)\right) \chi
$$

is an element of $\operatorname{Ch}(\mathcal{S})$ with $\varphi_{1}=p \varphi_{2}$.

By Lemma 2.6, we do not need to consider the $\mathbb{Q}$-classes of $G$ containing elements whose centralizers have orders coprime to $p$ when we determine the representation type of $R(G)_{p}$. We partition the remaining classes into $p^{\prime}$-sections.

Lemma 2.7. Let $p$ be a prime, and $x \in G$ be an element whose order is not divisible by $p$. Then, the function $\nu_{x}$ with

$$
\nu_{x}(g)= \begin{cases}1, & g_{p^{\prime}} \text { lies in the } \mathbb{Q} \text {-class of } x \\ 0, & \text { else }\end{cases}
$$

is contained in $R(G)_{p}$.

Proof. Let $g \in G$ with $g_{p^{\prime}}=x$, and $\mathcal{S}_{g}$ be the $\mathbb{Q}$-class of $g$. Then, $\mathcal{S}_{g}$ can be partitioned into conjugacy classes $C_{1}, \ldots, C_{k}$. Let $y_{1}, \ldots, y_{k} \in G$ be representatives of $C_{1}, \ldots, C_{k}$. For the function $\vartheta_{\mathcal{S}_{g}}$ of $G$ with $\vartheta_{\mathcal{S}_{g}}(y)=1$ for $y \in \mathcal{S}_{g}$ and $\vartheta_{\mathcal{S}_{g}}(y)=0$ 
for $y \in G \backslash \mathcal{S}_{g}$, we obtain

$$
\begin{aligned}
\vartheta_{\mathcal{S}_{g}} & =\sum_{i=1}^{k} \vartheta_{y_{i}}=\sum_{i=1}^{k} \frac{1}{\left|C_{G}(g)\right|} \sum_{\chi \in \operatorname{Irr}(G)} \chi\left(y_{i}^{-1}\right) \chi=\frac{1}{\left|C_{G}(g)\right|} \sum_{\chi \in \operatorname{Irr}(G)}\left(\sum_{i=1}^{k} \chi\left(y_{i}^{-1}\right)\right) \chi \\
& =\frac{1}{\left|C_{G}(g)\right|} \sum_{\chi \in \operatorname{Irr}(G)}\left(\sum_{\sigma \in \operatorname{Gal}\left(\mathbb{Q}\left(\operatorname{cl}_{G}\left(y_{1}\right)\right) / \mathbb{Q}\right)} \sigma\left(\chi\left(y_{1}^{-1}\right)\right)\right) \chi \\
& =\sum_{\chi \in \operatorname{Irr}(G)} \frac{\operatorname{Tr}_{\mathbb{Q}\left(\operatorname{cl}_{G}\left(y_{1}\right)\right) / \mathbb{Q}}\left(\chi\left(y_{1}^{-1}\right)\right)}{\left|C_{G}(g)\right|} \chi .
\end{aligned}
$$

The coefficients of the irreducible characters in this sum all lie in $\mathbb{Q}$, whence $\vartheta_{\mathcal{S}_{g}} \in$ $R(G)_{p}^{\prime}$.

Let $\mathcal{S}$ denote the rational $p^{\prime}$-section of $x$ in $G$. Then, $\mathcal{S}$ is a union of $\mathbb{Q}$-classes $\mathcal{S}_{1}, \ldots, \mathcal{S}_{\ell}$ of $G$. Thus, $\nu_{x}=\vartheta_{\mathcal{S}_{1}}+\ldots+\vartheta_{\mathcal{S}_{\ell}}$ where $\vartheta_{\mathcal{S}_{j}}(h)=1$ for $h \in \mathcal{S}_{j}$ and $\vartheta_{\mathcal{S}_{j}}(h)=0$ for $h \in G \backslash \mathcal{S}_{j}, j \in\{1, \ldots, \ell\}$. Since each $\vartheta_{\mathcal{S}_{j}}$ is contained in $R(G)_{p}^{\prime}, \nu_{x}$ also lies in $R(G)_{p}^{\prime}$.

Let $\varepsilon$ be an $r$ th root of unity where $r$ is the $p^{\prime}$-part of $|G|$, and $\mathfrak{p}$ be a maximal ideal in $\mathbb{Z}[\varepsilon]$ containing $p$. Then, the function $\tilde{\nu}_{x}$ given by $\tilde{\nu}_{x}(g)=1$ if $g_{p^{\prime}}$ is conjugate to $x$ in $G$ and $\tilde{\nu}_{x}(g)=0$ else lies in $(\mathbb{Z}[\varepsilon])_{\mathfrak{p}} \otimes R(G)$ by [29, Lemma 8.19]. Hence, for a transversal $\left\{\tilde{x}_{1}, \ldots, \tilde{x}_{r}\right\}$ of the conjugacy classes contained in the $\mathbb{Q}$-class of $x$, we get $\nu_{x}=\tilde{\nu}_{\tilde{x}_{1}}+\ldots+\tilde{\nu}_{\tilde{x}_{r}}$. This implies $\nu_{x} \in R(G)_{p}^{\prime} \cap\left((\mathbb{Z}[\varepsilon])_{\mathfrak{p}} \otimes R(G)\right)=R(G)_{p}$.

Remark 2.8. By Lemma 2.7, the function $\varphi \in \mathbb{Q} \otimes R(G)$ lies in $R(G)_{p}$ if and only if $\nu_{x} \varphi$ lies in $R(G)_{p}$ for any $p^{\prime}$-element $x \in G$. For rational $p^{\prime}$-sections $\mathcal{S}_{1}, \ldots, \mathcal{S}_{k}$, if

$\alpha_{1}, \beta_{1}$ generate the $R(G)_{p}$-module $\left(R(G)_{p}^{\prime} \cap \mathrm{Ch}_{\mathbb{Q}}\left(\mathcal{S}_{1}\right)\right) /\left(R(G)_{p} \cap \mathrm{Ch}_{\mathbb{Q}}\left(\mathcal{S}_{1}\right)\right)$,

$\alpha_{k}, \beta_{k}$ generate the $R(G)_{p}$-module $\left(R(G)_{p}^{\prime} \cap \mathrm{Ch}_{\mathbb{Q}}\left(\mathcal{S}_{k}\right)\right) /\left(R(G)_{p} \cap \mathrm{Ch}_{\mathbb{Q}}\left(\mathcal{S}_{k}\right)\right)$, then $\alpha_{1}+\ldots+\alpha_{k}$ and $\beta_{1}+\ldots+\beta_{k}$ generate the $R(G)_{p}$-module

$$
\left(R(G)_{p}^{\prime} \cap \mathrm{Ch}_{\mathbb{Q}}\left(\mathcal{S}_{1} \cup \ldots \cup \mathcal{S}_{k}\right)\right) /\left(R(G)_{p} \cap \mathrm{Ch}_{\mathbb{Q}}\left(\mathcal{S}_{1} \cup \ldots \cup \mathcal{S}_{k}\right)\right) .
$$

Analogously, the radical of $R(G)_{p}^{\prime} / R(G)_{p}$ is cyclic if and only if for each rational $p^{\prime}$-section $\mathcal{S}$, the $R(G)_{p}$-module $\operatorname{rad}\left(\left(R(G)_{p}^{\prime} \cap \mathrm{Ch}_{\mathbb{Q}}(\mathcal{S})\right) /\left(R(G)_{p} \cap \mathrm{Ch}_{\mathbb{Q}}(\mathcal{S})\right)\right)$ is cyclic. Thus, the representation type of $R(G)_{p}$ can be determined by considering the rational $p^{\prime}$-sections of $G$ separately.

These observations yield a necessary condition for $R(G)$ having finite representation type. 
Theorem 2.9. Let $p$ be a prime, and $\mathcal{S}$ be a rational $p^{\prime}$-section of $G$ containing $a+1$ different $\mathbb{Q}$-classes. Then, any generating system of the $R(G)_{p}$-module $R(G)_{p}^{\prime} / R(G)_{p}$ contains at least a elements.

Proof. Let $\varepsilon=\zeta_{\exp (G)}, \mathcal{C}_{1}, \ldots, \mathcal{C}_{a+1}$ be the $\mathbb{Q}$-classes in $\mathcal{S}$, and $x_{1} \in \mathcal{C}_{1}, \ldots, x_{a+1} \in$ $\mathcal{C}_{a+1}$. As in the proof of Lemma 2.7, we can show that there exist functions $\varphi_{j} \in$ $R(G)_{p}^{\prime}$ with $\varphi_{j}(x)=1$ for $x \in \mathcal{C}_{j}$ and $\varphi_{j}(x)=0$ for $x \in G \backslash \mathcal{C}_{j}, j=1, \ldots, a+1$. Certainly, the $\varphi_{j}$ do not lie in $R(G)_{p}$ since for $x_{j} \in \mathcal{C}_{j}$ and $x_{k} \in \mathcal{C}_{k}, j \neq k$, we have $\varphi_{j}\left(x_{j}\right) \not \equiv \varphi_{j}\left(x_{k}\right)(\bmod \mathfrak{p})$ for a maximal ideal $\mathfrak{p}$ of $\mathbb{Z}[\varepsilon]$ containing $p$.

We choose $\psi_{1}, \ldots, \psi_{n} \in R(G)_{p}^{\prime}$ such that $\psi_{1}+R(G)_{p}, \ldots, \psi_{n}+R(G)_{p}$ are generators of the $R(G)_{p}$-module $R(G)_{p}^{\prime} / R(G)_{p}$. Then, for any $j \in\{1, \ldots, a+1\}$, we can find $\lambda_{1, j}, \ldots, \lambda_{n+1, j} \in R(G)_{p}$ such that $\lambda_{1, j} \psi_{1}+\ldots+\lambda_{n, j} \psi_{n}+\lambda_{n+1, j}=\varphi_{j}$. We define

$$
F: R(G)_{p}^{\prime} \rightarrow(\mathbb{Z}[\varepsilon] / \mathfrak{p})^{a+1}, \quad \vartheta \mapsto\left(\vartheta\left(x_{1}\right)+\mathfrak{p}, \ldots, \vartheta\left(x_{a+1}\right)+\mathfrak{p}\right) .
$$

Then, $F\left(\varphi_{1}\right), \ldots, F\left(\varphi_{a+1}\right)$ are linearly independent in the $\mathbb{Z}[\varepsilon] / \mathfrak{p}$-vector space $(\mathbb{Z}[\varepsilon] / \mathfrak{p})^{a+1}$, i.e. $F\left(\varphi_{1}\right), \ldots, F\left(\varphi_{a+1}\right)$ form a basis of this vector space. Moreover, the $F\left(\lambda_{i, j}\right)$ induce multiplications with scalars in $(\mathbb{Z}[\varepsilon] / \mathfrak{p})^{a+1}$ since $\lambda_{i, j}(x) \equiv \lambda_{i, j}(y)$ $(\bmod \mathfrak{p})$ for $x, y \in \mathcal{S}$. Therefore, $F\left(\psi_{1}\right), \ldots, F\left(\psi_{n}\right), F(\mathbb{1})$ also generate the vector space $(\mathbb{Z}[\varepsilon] / \mathfrak{p})^{a+1}$ and we conclude $n \geq a$.

Corollary 2.10. Let $G$ have a rational $p^{\prime}$-section containing at least four $\mathbb{Q}$-classes for some prime $p$. Then, $R(G)$ has infinite representation type. In particular, the character ring of a group whose exponent is not cube-free has infinite representation type.

Next, we focus our attention on certain functions in $R(G)_{p}^{\prime}$ which are defined by their values on the $p$-parts of the elements of $G$.

Definition 2.11. Let $p$ be a prime dividing $|G|$. For a positive integer $i$, we define the class function $\mu_{i}$ by

$$
\mu_{i}(g):=\left\{\begin{array}{ll}
1, & \left|\left\langle g_{p}\right\rangle\right|=p^{i} \\
0, & \text { else }
\end{array} \quad \text { for } g \in G .\right.
$$

Lemma 2.12. Let $p$ be a prime dividing $|G|$, and $k$ be the largest integer such that $p^{k}$ divides $\exp (G)$. Then, $\mu_{i} \in R(G)_{p}^{\prime} \backslash R(G)_{p}$ for $i=0, \ldots, k$.

Proof. It is clear that $\mu_{i}$ lies in $R(G)_{p}^{\prime}$ for $i=1, \ldots, k$ since similarly to $\nu_{x}$ in Lemma 2.7, we can regard $\mu_{i}$ as a function on the $\mathbb{Q}$-classes of $G$. If $\mu_{i}$ was also contained in $R(G)_{p}$, then $\mu_{i}(g) \equiv \mu_{i}\left(g_{p^{\prime}}\right)(\bmod \mathfrak{p})$ would hold for any $g \in G$ where $\mathfrak{p}$ denotes a maximal ideal of $\mathbb{Z}\left[\zeta_{\exp (G)}\right]$ containing $p$. Now, there exists an element 
$x \in G$ of order $p^{i}$, whence $\mu_{i}(x) \equiv 1 \not \equiv 0 \equiv \mu_{i}(1)(\bmod \mathfrak{p})$ for $i>0$. Moreover, $G$ contains an element $y$ of order $p$ so that $\mu_{0}$ does also not lie in $R(G)$ because $\mu_{0}(y) \equiv 0 \not \equiv 1 \equiv \mu_{0}(1)(\bmod \mathfrak{p})$.

We conclude this section by proving a kind of an orthogonality relation for the characters of $G$. From this, we obtain functions in $R(G)^{\prime}$ which are nonzero only on a certain cyclic subgroup of $G$ (and its conjugates in $G$ ) or only on the generators of this subgroup (and their conjugates) respectively.

Lemma 2.13. Let $x \in G$, and $\varepsilon$ be an $|\langle x\rangle|$ th root of unity. Moreover, let $a_{\chi(x)}(\varepsilon)$ be the multiplicity of the linear character $\lambda \in \operatorname{Irr}(\langle x\rangle)$ in $\chi_{\langle x\rangle}$ such that $\lambda(x)=\varepsilon$. Then, for $g \in G$,

$$
\begin{aligned}
& \sum_{\chi \in \operatorname{Irr}(G)} a_{\chi(x)}(\varepsilon) \chi(g) \\
& = \begin{cases}\frac{\left|C_{G}(g)\right|}{|\langle x\rangle|} \cdot \sum_{\sigma \in \operatorname{Gal}\left(\mathbb{Q}\left(\zeta_{\langle\langle g\rangle|}\right) / \mathbb{Q}\left(\operatorname{cl}_{G}(g)\right)\right)} \sigma\left(\varepsilon^{k}\right), & g \sim_{G} x^{k} \text { for some } k \in \mathbb{Z} \\
0, & \text { else }\end{cases} \\
& =\left\{\begin{array}{ll}
\frac{\left|N_{G}(\langle g\rangle)\right| \cdot\left|C_{G}(x)\right|}{\left|N_{G}(\langle x\rangle)\right| \cdot|\langle x\rangle|} \sum_{\sigma \in \operatorname{Gal}\left(\mathbb{Q}(\zeta|\langle x\rangle|) / \mathbb{Q}\left(\operatorname{cl}_{G}(x)\right)\right)} \sigma\left(\varepsilon^{k}\right), & g \sim_{G} x^{k} \text { for some } k \in \mathbb{Z} \\
0, & \text { else. }
\end{array} .\right.
\end{aligned}
$$

Proof. Let $\lambda \in \operatorname{Irr}(\langle x\rangle)$ be the character with $\lambda(x)=\varepsilon$. Then,

$$
\begin{aligned}
& \sum_{\chi \in \operatorname{Irr}(G)} a_{\chi(x)}(\varepsilon) \chi(g)=\sum_{\chi \in \operatorname{Irr}(G)}\langle\chi, \lambda\rangle_{\langle x\rangle} \chi(g)=\sum_{\chi \in \operatorname{Irr}(G)} \frac{1}{|\langle x\rangle|} \sum_{h \in\langle x\rangle} \lambda(h) \chi\left(h^{-1}\right) \chi(g) \\
& =\frac{1}{|\langle x\rangle|} \sum_{h \in\langle x\rangle} \lambda(h) \sum_{\chi \in \operatorname{Irr}(G)} \chi\left(h^{-1}\right) \chi(g)=\frac{1}{|\langle x\rangle|} \sum_{\substack{h \in\langle x\rangle \\
h \sim_{G} g}} \lambda(h)\left|C_{G}(g)\right|
\end{aligned}
$$

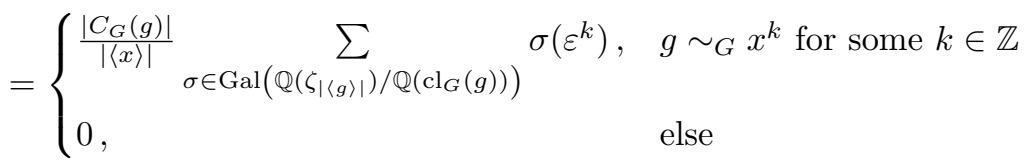

$$
\begin{aligned}
& = \begin{cases}\frac{\left|\operatorname{Gal}\left(\mathbb{Q}\left(\zeta_{\langle\langle g\rangle}\right) / \mathbb{Q}\left(\operatorname{cl}_{G}(g)\right)\right)\right|}{\left|\operatorname{Gal}\left(\mathbb{Q}\left(\zeta_{|\langle x\rangle|}\right) / \mathbb{Q}\left(\operatorname{cl}_{G}(x)\right)\right)\right|} \cdot \frac{\left|C_{G}(g)\right|}{|\langle x\rangle|} \cdot \sum_{\sigma \in \operatorname{Gal}\left(\mathbb{Q}\left(\zeta_{|\langle x\rangle|}\right) / \mathbb{Q}\left(\operatorname{cl}_{G}(x)\right)\right)} \sigma\left(\varepsilon^{k}\right), & g \sim_{G} x^{k} \text { for a } k \in \mathbb{Z} \\
0, & \text { else }\end{cases} \\
& = \begin{cases}\frac{\left|N_{G}(\langle g\rangle)\right| \cdot\left|C_{G}(x)\right|}{\left|N_{G}(\langle x\rangle)\right| \cdot|\langle x\rangle|} \cdot \sum_{\sigma \in \operatorname{Gal}\left(\mathbb{Q}(\zeta \backslash\langle x\rangle \mid) / \mathbb{Q}\left(\operatorname{cl}_{G}(x)\right)\right)} \sigma\left(\varepsilon^{k}\right), & g \sim_{G} x^{k} \text { for some } k \in \mathbb{Z} \\
0, & \text { else. }\end{cases}
\end{aligned}
$$




\section{Sylow subgroups of groups whose character rings have finite representation type}

We want to ascertain what the Sylow subgroups of $G$ can look like if $R(G)$ is representation-finite. We start with a trivial lemma which we will apply in several proofs to get reductions.

Lemma 3.1. Let $N$ be a normal subgroup of $G$. Then, for any prime $p$, the number of $\mathbb{Q}$-classes of p-elements in $G / N$ does not exceed the number of $\mathbb{Q}$-classes of $p$ elements in $G$. Moreover, if $x \in G$ is a p-element, then the $\mathbb{Q}$-class of $x$ in $G$ contains at least as many conjugacy classes as the $\mathbb{Q}$-class of $x N$ in $G / N$.

Proof. Clearly, if $g, x, y \in G$ are such that $g x g^{-1}=y$, then $g N x N g^{-1} N=$ $g x g^{-1} N=y N$. Thus, if $C_{1}, \ldots, C_{k}$ are the conjugacy classes of $G$, then $\bar{C}_{1}, \ldots, \bar{C}_{k}$ are the conjugacy classes of $G / N$ with $\bar{C}_{i}=\bar{C}_{j}$ for some $i \neq j$ possibly. Additionally, any $p$-element $\bar{x} \in G / N$ has a preimage $x \in G$ which is also a $p$-element.

For the abelian Sylow subgroups of $G$, it is easy to get a very restrictive assertion.

Theorem 3.2. Let $p$ be a prime and $P \in \operatorname{Syl}_{p}(G)$ be abelian. If $R(G)$ has finite representation type, then $P$ is cyclic of order $\leq p^{2}$ or elementary-abelian.

Proof. Suppose $P$ is neither cyclic of order $p^{2}$ nor elementary-abelian. We show that $G$ has at least four $\mathbb{Q}$-classes of $p$-elements in $G$.

Since $P$ is abelian, two elements of $P$ are conjugate in $G$ if and only if they are conjugate in $N_{G}(P)$. That is why we assume $P \unlhd G$. By Corollary $2.10, \exp (P) \leq p^{2}$ if $R(G)$ has finite representation type. Thus, $P$ is isomorphic to a direct product $A:=Z_{1} \times \ldots \times Z_{r}$ of cyclic groups $Z_{1}, \ldots, Z_{r}$ of order $p$ or $p^{2}$. In the following, we identify $A$ and $P$.

Suppose $\left|Z_{i}\right|=p$ and $\left|Z_{j}\right|=p^{2}$ for some $i, j \in\{1, \ldots, r\}$. W.l.o.g. let $i=1$ and $j=2$. We write $Z_{1}=\left\langle z_{1}\right\rangle$ and $Z_{2}=\left\langle z_{2}\right\rangle$. Then, there is no $x \in A$ such that $x^{p}=\left(z_{1}, 1, \ldots, 1\right)$ whereas $\left(1, z_{2}, 1, \ldots, 1\right)^{p}=\left(1, z_{2}^{p}, 1, \ldots, 1\right)$. Hence, $\left(z_{1}, 1, \ldots, 1\right)$ and $\left(1, z_{2}^{p}, 1, \ldots, 1\right)$ lie in different $\mathbb{Q}$-classes of $G$. This implies that the $\mathbb{Q}$-classes of $1,\left(z_{1}, 1, \ldots, 1\right),\left(1, z_{2}^{p}, 1, \ldots, 1\right)$ and $\left(1, z_{2}, 1, \ldots, 1\right)$ in $G$ are pairwise disjoint.

Finally, we assume $P \cong C_{p^{2}}^{n}$ for some integer $n$ with $n \geq 2$. Then, $P$ contains exactly $p^{2 n}-1-\left(p^{n}-1\right)=p^{n}\left(p^{n}-1\right)$ elements of order $p^{2}$, that means $P$ contains exactly $p^{n-1}\left(p^{n}-1\right) /(p-1)$ cyclic subgroups of order $p^{2}$. If $G$ has at most three $\mathbb{Q}$-classes of $p$-elements, then these cyclic subgroups are conjugate in $G$. Thus, $G$ acts transitively on the set $\mathcal{M}$ of cyclic subgroups of order $p^{2}$ of $G$. For $H \in \mathcal{M}$, this yields a bijection $G / \operatorname{stab}_{G}(H) \rightarrow \mathcal{M}$. Now, $P$ is contained in $\operatorname{stab}_{G}(H)$, so $p$ does not divide $\left|G: \operatorname{stab}_{G}(H)\right|$. On the other hand, $p$ divides $|\mathcal{M}|=p^{n-1}\left(p^{n}-1\right)(p-1)$. This contradicts $\left|G: \operatorname{stab}_{G}(H)\right|=|\mathcal{M}|$. 
3.1. Nonabelian composition factors. In this section, we consider the possible number and the possible isomorphism types of the nonabelian composition factors of a group $G$ whose character ring has finite representation type. Moreover, we will show that if $G$ has a component, then this must be a Hall subgroup of $G$.

As usual, we write $F(G)$ for the Fitting subgroup of $G$ and $E(G)$ for the layer of $G$. The generalized Fitting subgroup is denoted by $F^{*}(G)$. Moreover, we use the notation in the Atlas [8] for the finite simple groups except for the classical groups. We write $\operatorname{PSL}(n, q)$ for the projective special linear group of degree $n$ over a field of $q$ elements and $\operatorname{PSU}(n, q)$ for the projective special unitary group of degree $n$ over a field of $q^{2}$ elements. Finally, $\operatorname{Sp}(2 n, q)$ is the (nonprojective) symplectic group of degree $2 n$ over a field of $q$ elements.

Lemma 3.3. Let $G$ be a group of even order such that $R(G)$ has finite representation type. Then, there is only one class of involutions in $G$.

Proof. The conjugacy class of an involution coincides with its $\mathbb{Q}$-class in $G$. Hence, $G$ contains at most two conjugacy classes of involutions. Moreover, if $G$ has an element of order 4, then all involutions of $G$ are conjugate.

Let $P \in \operatorname{Syl}_{2}(G)$ with $\exp (P)=2$, i.e. $P$ is elementary-abelian. We assume that there are two classes of involutions in $G$. Then, there are also two classes $C_{1}, C_{2}$ of involutions in $N_{G}(P)$. For $x \in C_{1}$, we get $\left|C_{1}\right|=\left|N_{G}(P): C_{N_{G}(P)}(x)\right|$. Since $P \leq C_{N_{G}(P)}(x)$, this implies that $\left|C_{1}\right|$ is odd. However, $\left|C_{2}\right|$ is odd by the same argument, so $|P|=1+\left|C_{1}\right|+\left|C_{2}\right|$ is odd. This is impossible as $P$ is a nontrivial 2-group.

Lemma 3.4. Let $G$ be a nonsolvable group with at most three $\mathbb{Q}$-classes of 2 elements. Then, there is no normal subgroup in $G$ which is isomorphic to a direct product of two or more nonabelian simple groups.

Proof. Suppose $G$ possesses a normal subgroup $N=S_{1} \times \ldots \times S_{n}$ where each $S_{i}$ is a nonabelian simple group. Then, we find involutions $t_{1} \in S_{1}, \ldots, t_{n} \in S_{n}$ by the Theorem of Feit and Thompson. Certainly, the orders of the centralizers of $\left(t_{1}, \ldots, t_{n}\right)$ and $\left(t_{1}, \ldots, t_{n-1}, 1_{S_{n}}\right)$ in $G$ are different. Hence, $\left(t_{1}, \ldots, t_{n}\right)$ and $\left(t_{1}, \ldots, t_{n-1}, 1_{S_{n}}\right)$ are not conjugate in $G$.

Now, $G$ contains at least two conjugacy classes of involutions. Thus, there are no further classes of involutions and no element of order 4 in $G$ since $G$ has at most three classes of 2-elements. That is why $G$ contains exactly two classes of involutions and the Sylow 2-subgroups of $G$ are elementary-abelian. But then, the same arguments as in the proof of Lemma 3.3 would yield that there is some Sylow 2-subgroup of odd order in $G$. 
Proposition 3.5. Let $G$ be a group whose character ring has finite representation type. Then, $G$ has at most one nonabelian composition factor.

Proof. Suppose $G$ is not solvable. Since $R(G)$ has finite representation type, $G$ has at most three $\mathbb{Q}$-classes of 2-elements. Moreover, Lemma 3.1 yields that each of the groups $G / F(G),(G / F(G)) / F(G / F(G))$, etc. has also at most three $\mathbb{Q}$-classes of 2-elements.

Thus, we may assume that $F^{*}(G)=E(G)$ is a direct product of simple groups and $G$ contains at most three $\mathbb{Q}$-classes of 2-elements. By Lemma 3.4, $F^{*}(G)$ is simple, and since $C_{G}\left(F^{*}(G)\right) \leq F^{*}(G)$, we obtain $C_{G}\left(F^{*}(G)\right)=1$. Hence, $G / F^{*}(G)$ is isomorphic to a subgroup of $\operatorname{Out}\left(F^{*}(G)\right)$ which is solvable by the Schreier conjecture.

Now, we want to answer the question what a component of $G$ can look like if $R(G)$ is representation-finite. For this, we need two Lemmas which can be found in [44].

Lemma 3.6 (Yamaki). Let $G$ be a nonabelian simple group in which all involutions are conjugate. Then, $G$ is isomorphic to one of the following groups:

(1) Alternating groups: $A_{5}, A_{6}, A_{7}$,

(2) Groups of Lie-Type: $\operatorname{PSL}(2, q), \operatorname{PSL}(3, q), \operatorname{PSL}(4, q), \operatorname{PSU}(3, q), \operatorname{PSU}(4, q)$, ${ }^{3} D_{4}(q), G_{2}(q),{ }^{2} G_{2}\left(3^{2 n+1}\right),{ }^{2} B_{2}\left(2^{2 n+1}\right)$,

(3) Sporadic groups: $M_{11}, M_{22}, M_{23}, J_{1}, J_{3}, M c L, O^{\prime} N, L y, T h$.

Lemma 3.7 (Yamaki). There exists no finite simple group $G$ such that $G$ has more than one conjugacy class of involutions and all involutions in $G$ are conjugate under $\operatorname{Aut}(G)$.

Theorem 3.8. Let $N$ be a nonabelian quasisimple normal subgroup of a group $G$ whose character ring has finite representation type. Then, $\operatorname{gcd}(|N|,|G: N|)=1$ and $N$ is isomorphic to one of the following groups:

(1) $\operatorname{PSL}(2, q)$ where $q>2$ is even and $q^{2}-1$ is cube-free,

(2) $\operatorname{PSL}(2, q)$ where $q>3$ is odd and none of the integers $q, q+1$ and $q-1$ is divisible by 16 or the third power of an odd prime,

(3) $\mathrm{SL}(2, p)$ where $p>2$ is an odd prime and $p+1$ as well as $p-1$ are cube-free,

(4) $A_{7}$,

(5) $J_{1}$.

Proof. At first, we assume that $N$ is simple and consider the isomorphism type of $N$. By the Lemmas 3.3 and 3.7, we conclude that $N$ is one of the simple groups in the list of Lemma 3.6. Moreover, the involutions of $N$ and $G \backslash N$ are not conjugate, so Lemma 3.3 yields that there is no involution in $G \backslash N$. In addition, if $N$ contains 
an element of order 4 , then $G / N$ has odd order since otherwise, $G$ would have four $\mathbb{Q}$-classes of 2-elements in contradiction to Corollary 2.10.

Now, we go through the list of Lemma 3.6 step by step.

- $A_{5}, A_{6}, A_{7}$

Each of these groups occurs in the list above since $A_{5} \cong \operatorname{PSL}(2,5)$ and $A_{6} \cong$ $\operatorname{PSL}(2,9)$.

- $\operatorname{PSL}(2, q)$

Let $N \cong \operatorname{PSL}(2, q)$ with $q$ even. Then $N$ contains elements of order $q-1$ and $q+1$ respectively. Hence, $q-1$ and $q+1$, or equivalently $q^{2}-1$, must be cube-free.

We set $N \cong \operatorname{PSL}(2, q)$ with $q=p^{k}$ where $p$ is odd. Then, there are elements of order $(q-1) / 2$ and $(q+1) / 2$ respectively in $N$. Thus, $q+1$ and $q-1$ are not divisible by 16 or the third power of a prime.

Suppose $k \geq 3$. Then, $N$ contains exactly two conjugacy classes of elements of order $p$. We choose representatives $x$ and $y$ of these classes. The automorphism group of $N$ is described in [43] for instance. We obtain that $G$ contains a subgroup isomorphic to $\operatorname{PGL}(2, q)$ if $x$ and $y$ are conjugate in $G$. However, the image of the diagonal matrix $\operatorname{diag}(1,-1) \in \mathrm{GL}(2, q)$ in $\operatorname{PGL}(2, q)$ has order 2. Hence, $\operatorname{PGL}(2, q)$ has two classes of involutions and only one of them contains elements of $N$. Consequently, $G$ has also two classes of involutions in contradiction to the assertion of Lemma 3.3. That is why $x$ and $y$ lie in different conjugacy classes of $G$.

If $q \equiv 1(\bmod 4)$, the character table of $\operatorname{PSL}(2, q)$ shows $\chi(1) \equiv \chi(x) \equiv \chi(y)$ $\left(\bmod p^{2}\right)$ for any $\chi \in \operatorname{Irr}(N)$, so $\chi(1) \equiv \chi(x) \equiv \chi(y)\left(\bmod p^{2}\right)$ for any $\chi \in \operatorname{Irr}(G)$. Therefore, the functions $\varphi_{1}$ and $\varphi_{2}$ with

$$
\varphi_{1}(g)=\left\{\begin{array}{ll}
p, & g \sim x \\
p, & g \sim y \\
0, & \text { else }
\end{array} \quad \text { and } \quad \varphi_{2}(g)= \begin{cases}p, & g \sim x \\
-p, & g \sim y \\
0, & \text { else }\end{cases}\right.
$$

lie in $\operatorname{rad}\left(R(G)_{p}^{\prime}\right) \backslash R(G)_{p}$.

Suppose there exist $\alpha \in \operatorname{rad}\left(R(G)_{p}^{\prime}\right)$ and $\eta_{1}, \eta_{2}, \psi_{1}, \psi_{2} \in R(G)_{p}$ such that $\varphi_{1}=$ $\eta_{1} \alpha+\psi_{1}$ and $\varphi_{2}=\eta_{2} \alpha+\psi_{2}$. Then, $\varphi_{2}(x) \not \equiv \varphi_{2}(y)\left(\bmod p^{2}\right)$ implies $\alpha(x) \not \equiv \alpha(y)$ $\left(\bmod p^{2}\right)$ since $\eta_{2}(x) \equiv \eta_{2}(y)\left(\bmod p^{2}\right)$ and $\psi_{2}(x) \equiv \psi_{2}(y)\left(\bmod p^{2}\right)$. On the other hand, we have $\left(\varphi_{1}-\psi_{1}\right)(x) \equiv\left(\varphi_{1}-\psi_{1}\right)(y)\left(\bmod p^{2}\right)$. Now, $\eta_{1} \alpha(x) \equiv \eta_{2} \alpha(y)$ $\left(\bmod p^{2}\right)$ which yields that $p$ divides $\eta_{1}(x)$ and $\eta_{1}(y)$. But then, $\eta_{1} \alpha(1) \equiv \eta_{1} \alpha(x)$ $\left(\bmod p^{2}\right)$. However, $\left(\varphi_{1}-\psi_{1}\right)(1) \not \equiv\left(\varphi_{1}-\psi_{1}\right)(x)\left(\bmod p^{2}\right)$ because $\psi_{1}(1)-\psi_{1}(x)$ is divisible by $p^{2}$ whereas $\varphi_{1}(1) \not \equiv \varphi_{1}(x)\left(\bmod p^{2}\right)$. Consequently, $\operatorname{rad}\left(R(G)_{p}^{\prime} / R(G)_{p}\right)$ is not cyclic, so $R(G)$ would have infinite representation type.

We can argue similarly in the case $q \equiv 3(\bmod 4)$. Let $\mathfrak{p}$ be the maximal ideal in $\mathcal{O}_{\mathbb{Q}(\sqrt{p})}$ containing $p$. Then, $\chi(1) \equiv \chi(x) \equiv \chi(y)\left(\bmod \mathfrak{p}^{3}\right)$ for any $\chi \in \operatorname{Irr}(N)$. 
Obviously, the same congruences hold for $\chi \in \operatorname{Irr}(G)$. Thus, the functions $\varphi_{1}$ and $\varphi_{2}$ with

$$
\varphi_{1}(g)=\left\{\begin{array}{ll}
p, & g \sim x \\
p, & g \sim y \\
0, & \text { else }
\end{array} \quad \text { and } \quad \varphi_{2}(g)= \begin{cases}\sqrt{-p}, & g \sim x \\
-\sqrt{-p}, & g \sim y \\
0, & \text { else }\end{cases}\right.
$$

lie in $\operatorname{rad}\left(R(G)_{p}^{\prime}\right) \backslash R(G)_{p}$. Analogously to the case $q \equiv 1(\bmod 4)$, we can show that there exist no $\alpha \in \operatorname{rad}\left(R(G)_{p}^{\prime}\right)$ and $\eta_{1}, \eta_{2}, \psi_{1}, \psi_{2} \in R(G)_{p}$ such that $\varphi_{1}=\eta_{1} \alpha+\psi_{1}$ and $\varphi_{2}=\eta_{2} \alpha+\psi_{2}$. This implies that $\operatorname{rad}\left(R(G)_{p}^{\prime} / R(G)_{p}\right)$ is not cyclic, so the representation type of $R(G)$ would be infinite.

- $\operatorname{PSL}(3, q)$ and $\operatorname{PSU}(3, q)$

The character tables of $\operatorname{PSL}(3, q)$ and $\operatorname{PSU}(3, q)$ have been determined in [39]. If $q$ is odd, then $\operatorname{PSL}(3, q)$ and $\operatorname{PSU}(3, q)$ contain elements of order 8 . Hence, $N$ cannot be isomorphic to one of these groups. Let $q$ be even. Since $\operatorname{PSU}(3,2)$ is solvable and $\operatorname{PSL}(3,2) \cong \operatorname{PSL}(2,7)$, we may assume $q>2$. Then, the Sylow 2-subgroups of $\operatorname{PSU}(3, q)$ are Suzuki 2-groups of type $B$. We will show in Lemma 3.16 that the character ring of a group whose Sylow 2-subgroups are Suzuki 2-groups of type $B$, $C$, or $D$ has infinite representation type. Thus, we only have to consider the groups $\operatorname{PSL}(3, q), q>2$ even.

At first, let $N \cong \operatorname{PSL}(3, q)$ with $q \equiv 2(\bmod 3)$. Moreover, let $\zeta$ be a root of unity of order $q-1$. Then, the elements $x, y, z \in N$ corresponding to the images of the diagonal matrices $X=\operatorname{diag}\left(\zeta, \zeta, \zeta^{-2}\right), Y=\operatorname{diag}\left(\zeta, \zeta^{2}, \zeta^{-3}\right)$ and $Z=\operatorname{diag}\left(\zeta, \zeta^{-1}, 1\right)$ in $\operatorname{PSL}(3, q)$ lie in three different $\mathbb{Q}$-classes of $N$. Now, $\left|C_{N}(x)\right|$ is even whereas $\left|C_{N}(y)\right|$ and $\left|C_{N}(z)\right|$ are odd, so $x$ is neither $\mathbb{Q}$-conjugate to $y$ nor to $z$ in $G$. Moreover, $z$ and $z^{-1}$ are conjugate in $N$ while $y$ and $y^{-1}$ lie in different conjugacy classes of $N$. Since $N$ contains elements of order $4,|G: N|$ is odd. This implies that $y$ and $z$ are not $\mathbb{Q}$-conjugate in $G$, so $G$ has at least three $\mathbb{Q}$-classes of elements of order $q-1$. For some prime $\ell$ dividing $q-1$, this yields that $G$ contains at least four $\mathbb{Q}$-classes of $\ell$-elements.

Next, we suppose $N \cong \operatorname{PSL}(3, q)$ with $q \equiv 1(\bmod 3)$. Then, $N$ has three $\mathbb{Q}$ classes of elements of order 4 . These classes must form a single $\mathbb{Q}$-class in $G$. The outer automorphisms of $N$ can be found in [43]. We obtain that $G$ contains a subgroup isomorphic to $\operatorname{PGL}(3, q)$.

We identify $N$ with $\operatorname{PSL}(3, q)$. The elements

$$
X:=\left(\begin{array}{ccc}
1 & & \\
& \omega & \\
& & \omega^{2}
\end{array}\right), \quad Y:=\left(\begin{array}{ccc}
\omega & & \\
& \omega & \\
& & \omega^{2}
\end{array}\right), \quad \text { and } \quad Z:=\left(\begin{array}{ccc}
\omega & 1 & \\
& \omega & \\
& & \omega^{2}
\end{array}\right)
$$


lie in $\operatorname{GL}(3, q)$ for some $1 \neq \omega \in \mathbb{F}_{q}$ with $\omega^{3}=1$. Obviously, their images $\bar{X}, \bar{Y}, \bar{Z}$ in $\operatorname{PGL}(3, q)$ are different. Moreover, these images are pairwise not $\mathbb{Q}$-conjugate in $\operatorname{PGL}(3, q)$, since $Z$ is not $\mathbb{Q}$-conjugate to any diagonal matrix in $\operatorname{GL}(3, q)$ (see [40]), and $\operatorname{det}(X)=1 \neq \operatorname{det}(Y)$.

Certainly, the $\mathbb{Q}$-class of $\bar{X}$ in $G$ contains exactly the elements of order 3 in $N$. Thus, $\bar{X}$ cannot be $\mathbb{Q}$-conjugate to $\bar{Y}$ or $\bar{Z}$ in $G$. If $\bar{Y}$ and $\bar{Z}$ were also not $\mathbb{Q}$ conjugate in $G$, then $G$ would contain at least four $\mathbb{Q}$-classes of 3-elements. On the other hand, if $\bar{Y}$ and $\bar{Z}$ were $\mathbb{Q}$-conjugate in $G$, then $G / N$ would act transitively on the set of the $\mathbb{Q}$-classes of $\bar{Y}$ and $\bar{Z}$. But then, $|G / N|$ would be even which is impossible since $N$ contains elements of order 4 .

- $\operatorname{PSL}(4, q)$ and $\operatorname{PSU}(4, q)$

For $q$ odd, the groups $\operatorname{PSL}(4, q)$ and $\operatorname{PSU}(4, q)$ contain elements of order 8 . Hence, $N$ is not isomorphic to one of these groups. Moreover, if $q$ is even, then there exist two conjugacy classes of involutions in $\operatorname{PSL}(4, q)$ and $\operatorname{PSU}(4, q)$ respectively $[17,18]$. The Lemmas 3.7 and 3.3 now yield that $N$ is not isomorphic to such a group.

- ${ }^{3} D_{4}(q)$

The conjugacy classes of the groups ${ }^{3} D_{4}(q)$ have been determined in [13]. In particular, we learn from this article that ${ }^{3} D_{4}(q)$ contains elements of order 8 . Thus, $N \nsubseteq{ }^{3} D_{4}(q)$.

- $G_{2}(q)$

The groups $G_{2}(q)$ also possess elements of order 8 as we can detect from [33] (for $q$ odd) and [9] (for $q$ even). Thus, $N \neq G_{2}(q)$.

- ${ }^{2} G_{2}\left(3^{2 n+1}\right)$

Suppose $N \cong{ }^{2} G_{2}\left(3^{2 n+1}\right)$. Then, the Sylow 3 -subgroups of $N$ are nonabelian of exponent 9, and two Sylow 3-subgroups $P_{1}, P_{2} \in \operatorname{Syl}_{3}(N)$ either coincide or intersect trivially [42]. Additionally, $Z\left(P_{1}\right)$ and $P_{1} \backslash Z\left(P_{1}\right)$ contain elements of order 3. Consequently, $G$ would have at least four $\mathbb{Q}$-classes of 3-elements, so the representation type of $R(G)$ would be infinite.

- ${ }^{2} B_{2}\left(2^{2 n+1}\right)$

Suppose $N \cong{ }^{2} B_{2}\left(2^{2 n+1}\right)$ and $P \in \operatorname{Syl}_{2}(N)$. By [41], we get $|P|=|Z(P)|^{2}$ and the involutions of $P$ are permuted transitively by a cyclic subgroup of $N$. This implies that $P$ is a Suzuki 2-group of type $A$ [26]. In Lemma 3.15, we will show that $R(H)$ has infinite representation type if $H$ is a finite group whose Sylow 2subgroups are Suzuki 2-groups of type $A$. Since $|G / N|$ is odd, $R(G)$ would have infinite representation type. 
- Sporadic groups

Let $N$ be isomorphic to a sporadic group in the list of Lemma 3.6. Each of the groups $M_{11}, M_{22}, M_{23}, J_{3}, M c L, O^{\prime} N, L y$, and $T h$ possesses elements of order 8 . Hence, $N \cong J_{1}$.

After considering simple groups $N$, we now assume that $N$ is quasisimple with $Z(N) \neq 1$. For a prime $p$, Lemma 3.1 yields that $N / Z(N)$ has at most as many $\mathbb{Q}$-classes of $p$-elements as $N$ does. That is why $N / Z(N)$ is isomorphic to $\operatorname{PSL}(2, q)$, $A_{7}, J_{1}, \operatorname{PSU}(3, q)$ ( $q>2$ even), or ${ }^{2} B_{2}\left(2^{2 n+1}\right)$.

The Schur multipliers of $\operatorname{PSL}\left(2,2^{n}\right)(n>2), J_{1}$, and ${ }^{2} B_{2}\left(2^{2 n+1}\right)(n>1)$ are trivial. Thus, $N / Z(N)$ cannot be isomorphic to one of these groups. The Schur multiplier of ${ }^{2} B_{2}(8)$ is isomorphic to $C_{2}^{2}$. If $N / Z(N) \cong{ }^{2} B_{2}(8)$ and $Z(N) \neq 1$, then $G$ would possess more than three $\mathbb{Q}$-classes of 2 -elements. Similarly, for some even prime power $q>2$, a central extension of $\operatorname{PSU}(3, q)$ has either Sylow 2subgroups which are Suzuki 2-groups of type $B$ or contains more than three $\mathbb{Q}$ classes of 2-elements. Since $\operatorname{PSL}(2,4) \cong \operatorname{PSL}(2,5)$, it remains to consider the cases $N / Z(N) \cong A_{7}$ and $N / Z(N) \cong \operatorname{PSL}(2, q)$ with $q>3$ odd.

Suppose $N / Z(N) \cong A_{6}$ or $A_{7}$. Then, $N / Z(N)$ contains two $\mathbb{Q}$-classes of elements of order 2 and 3 respectively. Moreover, the Schur multiplier of $N / Z(N)$ is isomorphic to $C_{6}$. If both of the $\mathbb{Q}$-classes of elements of order 3 in $N$ were conjugate in $G$, then $|G / N|$ would be even. But this implies that $G$ has at least four $\mathbb{Q}$-classes of 2-elements since $A_{6}$ as well as $A_{7}$ contain elements of order 4 . Hence, $G$ would contain more than three $\mathbb{Q}$-classes of 2-elements or 3-elements respectively if $Z(N) \neq 1$ and $N / Z(N) \cong A_{6}$ or $A_{7}$.

Suppose $N / Z(N) \cong \operatorname{PSL}(2, q)$ with $q>3$ odd. Since $\operatorname{PSL}(2,9) \cong A_{6}$, we may assume $q \neq 9$. Then, the Schur multiplier of $N / Z(N)$ is isomorphic to $C_{2}$, whence $N \cong \operatorname{SL}(2, q)$. If $q=p^{k}$ with $k \geq 3$, then the same arguments as for $\operatorname{PSL}(2, q)$ would yield that $R(G)$ has infinite representation type. Furthermore, $\operatorname{SL}(2, q)$ contains elements of order $q+1$ and $q-1$ respectively, so $q+1$ and $q-1$ must be cube-free. However, $p^{2}-1$ is divisible by 8 for any odd prime $p$. Thus, we conclude that $q$ is already prime.

Lastly, we show $\operatorname{gcd}(|N|,|G: N|)=1$. Since $N$ is isomorphic to one of the groups $\operatorname{PSL}(2, q), \operatorname{SL}(2, p), A_{7}$ or $J_{1}$, there is no nontrivial element of odd order in $N$ which centralizes a Sylow 2-subgroup of $N$. As already mentioned, $G \backslash N$ does not contain any involution.

Let $p$ be an odd prime and suppose that there exist $p$-elements $x \in N$ and $y \in G \backslash N$. If $y$ commutes with every element of $N$, then $y$ and $x y$ lie in different $\mathbb{Q}$ classes of $G$ because $x y$ does not centralize any Sylow 2-subgroup of $G$. Certainly, 
neither $y$ nor $x y$ is conjugate to a power of $x$. Hence, $G$ would have at least four $\mathbb{Q}$-classes of $p$-elements.

Obviously, there is also no element of order 4 in $G \backslash N$ which centralizes $N$ since its square would lie in $N$. Thus, any element of $G \backslash N$ whose order is not prime to $|N|$ acts nontrivially on $N$ by conjugation.

As is well known, $\operatorname{Out}\left(J_{1}\right)=1$ and $\operatorname{Out}\left(A_{7}\right) \cong C_{2}$. Since $A_{7}$ possesses elements of order 4, we conclude immediately $G=N$ if $N$ is isomorphic to $J_{1}$ or $A_{7}$.

Let $\ell$ be an odd prime and $N \cong \operatorname{PSL}\left(2, \ell^{f}\right)$. Then, the outer automorphism group of $N$ has order $2 f$. We have already seen that $f \leq 2$ and $G$ has no subgroup isomorphic to $\operatorname{PGL}\left(2, \ell^{f}\right)$. Hence, for $f=1$, there is no element in $G \backslash N$ acting nontrivially on $N$. Moreover, if $f=2$, then any element of $G \backslash N$ which acts nontrivially on $N$ would be a 2-element. However, $N$ contains elements of order 4 in this case. That is why $|G: N|$ is odd.

Likewise, there is no element in $G \backslash N$ acting nontrivially on $N$ if $N \cong \operatorname{SL}(2, \ell)$, since any nontrivial automorphism of odd order of $\operatorname{SL}(2, \ell)$ induces a nontrivial automorphism of odd order of $\operatorname{PSL}(2, \ell)$.

Finally, suppose $N \cong \operatorname{PSL}\left(2,2^{f}\right)$. Then, the outer automorphism group of $N$ is a cyclic group of order $f$. We have to exclude that $p$ divides $f$ and one of $2^{f}+1$ or $2^{f}-1$. We show that in this case, $2^{f}+1$ or $2^{f}-1$ is even divisible by $p^{2}$. Hence, $G$ would have at least four $\mathbb{Q}$-classes of $p$-elements.

Let $p$ divide $f$ and $2^{f}-1$. Then, there exists a positive integer $k$ such that $f=k p$. Thus, $2^{k} \equiv\left(2^{k}\right)^{p} \equiv 2^{f} \equiv 1(\bmod p)$. That is why the first and the second factor of

$$
2^{k p}-1=\left(2^{k}-1\right) \cdot\left(2^{k(p-1)}+2^{k(p-2)}+\ldots+2^{k}+1\right)=\left(2^{k}-1\right) \cdot \sum_{i=0}^{p-1}\left(2^{k}\right)^{i}
$$

are both divisible by $p$, whence $p^{2}$ divides $2^{f}-1$.

Now, let $p$ divide $f$ and $2^{f}+1$. Clearly, we can find a positive integer $k$ such that $f=k p$. Then, $-2^{k} \equiv\left(-2^{k}\right)^{p} \equiv-2^{f} \equiv 1(\bmod p)$. Again, both factors of

$$
2^{k p}+1=\left(2^{k}+1\right) \cdot\left(2^{k(p-1)}-2^{k(p-2)}+-\ldots-2^{k}+1\right)=\left(2^{k}+1\right) \cdot \sum_{i=0}^{p-1}\left(-2^{k}\right)^{i}
$$

are divisible by $p$, so $p^{2}$ divides $2^{f}+1$.

Thus, we have excluded all cases with $\operatorname{gcd}(|N|,|G: N|)>1$ and the theorem is proved.

Corollary 3.9. Let $G$ be a group such that $R(G)$ has finite representation type, and $N$ be a nonabelian quasisimple normal subgroup of $G$. Let $p$ be an odd prime which divides $|N|$. Then, the Sylow p-subgroups of $G$ are abelian. 
Proof. By Theorem 3.8, the Sylow $p$-subgroups of $G$ coincide with the Sylow $p$ subgroups of $N$. That is why we just go through the list of Theorem 3.8. The Sylow $p$-subgroups of $\operatorname{PSL}(2, q)$ are elementary-abelian or cyclic depending on whether $q$ is a power of $p$ or not. Likewise, the Sylow $p$-subgroups of $\operatorname{SL}(2, q)$ are cyclic if $q$ is prime. Moreover, it is well known that the Sylow $p$-subgroups of $A_{7}$ and $J_{1}$ are also abelian.

The character rings of the groups in the list of Theorem 3.8 have indeed finite representation type. For a group $G$ of this list, Theorem 4.6, which we will prove in Section 4.1, yields that $R(G)_{p}$ has finite representation type if the Sylow $p$ subgroups of $G$ are cyclic. Hence, we only have to consider such primes $p$ for which the Sylow $p$-subgroups of $G$ are not cyclic.

Let $G$ be any group of the list in Theorem 3.8, and $p$ be a prime such that the Sylow $p$-subgroups of $G$ are not cyclic. Looking at the character table of $G$, we observe that any rational $p^{\prime}$-section of $G$ has at most two $\mathbb{Q}$-classes and each $\mathbb{Q}$-class is already a conjugacy class (see Appendix respectively [8] for $J_{1}$ ). Moreover, it is easy to show that $\operatorname{rad}\left(R(G)_{p}^{\prime} / R(G)_{p}\right)$ is cyclic for $G \cong \operatorname{PSL}\left(2, p^{2}\right)$ and that $\operatorname{rad}\left(R(G)_{2}^{\prime} / R(G)_{2}\right)$ is cyclic for $G \cong \operatorname{PSL}\left(2, p^{f}\right)$ with $f \in\{1,2\}$. Finally, $\operatorname{rad}\left(R\left(A_{7}\right)_{2}^{\prime} / R\left(A_{7}\right)_{2}\right)$ and $\operatorname{rad}\left(R\left(A_{7}\right)_{3}^{\prime} / R\left(A_{7}\right)_{3}\right)$ are cyclic as well. This yields the following assertion.

Corollary 3.10. The quasisimple groups whose character rings have finite representation type are exactly the following:

(1) $\operatorname{PSL}(2, q)$ where $q>2$ is even and $q^{2}-1$ is cube-free,

(2) $\operatorname{PSL}(2, q)$ where $q>3$ is odd and none of the integers $q, q+1$ and $q-1$ is divisible by 16 or the third power of an odd prime,

(3) $\mathrm{SL}(2, p)$ where $p>2$ is an odd prime and $p+1$ as well as $p-1$ are cube-free,

(4) $A_{7}$,

(5) $J_{1}$.

Theorem 3.8 states what a component of a nonsolvable group $G$ can look like if $R(G)$ is representation-finite. One may ask the same question for the nonabelian composition factor of $G$. With similar arguments as in the proof of Theorem 3.8, one can show that such a composition factor is isomorphic to one of the groups in the list of this theorem or $\operatorname{PSL}\left(2, p^{k}\right)$ with $k>2$. The author expects that there is no group $G$ with a composition factor isomorphic to $\operatorname{PSL}\left(2, p^{k}\right)$ with $k>2$ such that $R(G)$ is representation-finite, but has not been able to prove this yet.

3.2. Sylow 2-subgroups. Now, we want to find a list of 2-groups which can occur as Sylow 2-subgroups of $G$ if $R(G)$ has finite representation type. We need to introduce some more notations for the statement of our results. We call a group 
homocyclic if it is a direct product of cyclic groups of the same order. As usual, $D_{8}$ and $Q_{8}$ stand for the dihedral and the quaternion group of order 8 respectively. Moreover, we recall that a Suzuki 2-group $P$ is a nonabelian 2-group with more than one involution whose automorphism group has a cyclic subgroup which permutes the involutions of $P$ transitively. Higman has classified the Suzuki 2-groups and showed $\Omega_{1}(P)=Z(P)=P^{\prime}=\Phi(P)$ for any Suzuki 2-group $P[26]$. Here, $\Phi(P)$ is the Frattini subgroup of $P$ and $\Omega_{1}(P)$ is the subgroup of $P$ generated by all involutions of $P$. Furthermore, one can show that Suzuki 2-groups are resistant [11].

In several of the following proofs we use the classification of finite transitive linear groups by Huppert and Hering [25, 28].

Theorem 3.11 (Huppert, Hering). Let $p$ be a prime, $n$ be a positive integer, and $H \leq \mathrm{GL}(n, p)$ act transitively on the elements of $\mathbb{F}_{p}^{n} \backslash\{0\}$. Then, $H$ is isomorphic to a group $G$ which satisfies one of the following:

(1) $G \leq \Gamma \mathrm{L}\left(p^{n}\right)$

(2) $\mathrm{SL}(k, q) \unlhd G$ and $p^{n}=q^{k}$ for some $k \in \mathbb{N}$ with $k>1$,

(3) $\operatorname{Sp}(k, q) \unlhd G$ and $p^{n}=q^{k}$ for some $k \in 2 \mathbb{N}$,

(4) $G_{2}(q)^{\prime} \unlhd G, p=2$ and $2^{n}=q^{6}$,

(5) $p=3, n=2$ and $Q_{8} \unlhd G$,

(6) $n=2, p \in\{5,7,11,23\}$ and $\mathrm{SL}(2,3) \unlhd G$,

(7) $n=2, p \in\{11,19,29,59\}$ and $\mathrm{SL}(2,5) \unlhd G$,

(8) $p=3, n=4$ and either $\mathrm{SL}(2,5) \unlhd G$ or $S \unlhd G$ where $S$ is extraspecial of order 32 ,

(9) $p=2, n=4$ and $G \cong A_{6}$ or $G \cong A_{7}$,

(10) $p=3, n=6$ and $G \cong \mathrm{SL}(2,13)$,

(11) $p=2, n=6$ and $G \cong \operatorname{PSU}(3,3)$.

Here, the semilinear group

$$
\begin{aligned}
\Gamma L\left(p^{n}\right)=\{ & f \in \operatorname{Hom}\left(\mathbb{F}_{p^{n}}, \mathbb{F}_{p^{n}}\right): f(x)=a \sigma(x) \text { for suitable } a \in \mathbb{F}_{p^{n}}^{\times} \text {and } \\
& \left.\sigma \in \operatorname{Gal}\left(\mathbb{F}_{p^{n}} / \mathbb{F}_{p}\right)\right\}
\end{aligned}
$$

comes into play. This group has a normal subgroup

$$
\Gamma L_{0}\left(p^{n}\right)=\left\{f \in \operatorname{Hom}\left(\mathbb{F}_{p^{n}}, \mathbb{F}_{p^{n}}\right): f(x)=a x \text { for some } a \in \mathbb{F}_{p^{n}}^{\times}\right\} \cong \mathbb{F}_{p^{n}}^{\times}
$$

with factor group $\Gamma \mathrm{L}\left(p^{n}\right) / \Gamma \mathrm{L}_{0}\left(p^{n}\right) \cong \operatorname{Gal}\left(\mathbb{F}_{p^{n}} / \mathbb{F}_{p}\right)$. Hence, $\Gamma \mathrm{L}\left(p^{n}\right)$ is isomorphic to the semidirect product $\mathbb{F}_{p^{n}}^{\times} \rtimes \operatorname{Gal}\left(\mathbb{F}_{p^{n}} / \mathbb{F}_{p}\right)$. Additionally, we will be interested in the subgroups of $\Gamma \mathrm{L}\left(p^{n}\right)$ acting transitively on the one-dimensional subspaces of $\mathbb{F}_{p}^{n}$. 
Lemma 3.12. Let $p$ be a prime, $n \in \mathbb{N}$, and $G$ be a subgroup of $\Gamma \mathrm{L}\left(p^{n}\right)$ acting transitively on the one-dimensional subspaces of $\mathbb{F}_{p}^{n}$. For any prime $p_{i}$ which divides $d:=\operatorname{gcd}\left(\left(p^{n}-1\right) /(p-1), p-1\right)$, let $r_{i} \in \mathbb{Z}$ be the largest number such that $p_{i}^{r_{i}}$ divides $p-1$. Then, $\exp (G)$ is divisible by

$$
\frac{p^{n}-1}{p-1} \cdot \prod_{\substack{p_{i} \in \mathbb{P} \\ p_{i} \mid d}} p_{i}^{r_{i}}
$$

Proof. The action of $\Gamma L_{0}\left(p^{n}\right)$ on $\mathbb{F}_{p}^{n}$ corresponds to the conjugation action of the group

$$
H:=\left\{\left(\begin{array}{ll}
a & 0 \\
0 & 1
\end{array}\right): a \in \mathbb{F}_{p^{n}}^{\times}\right\} \quad \text { on the group } V:=\left\{\left(\begin{array}{ll}
1 & x \\
0 & 1
\end{array}\right): x \in \mathbb{F}_{p^{n}}\right\} .
$$

The elements of $H$ whose orders divide $p-1$ correspond to multiplications with elements from $\mathbb{F}_{p}^{\times}$. Hence, an element of a proper subgroup of $H$ whose order is no multiple of

$$
\frac{p^{n}-1}{p-1} \cdot \prod_{\substack{p_{i} \in \mathbb{P} \\ p_{i} \mid d}} p_{i}^{r_{i}}
$$

cannot map the element $X:=\left(\begin{array}{ll}1 & 1 \\ 0 & 1\end{array}\right)$ on an element of the form $Y^{j}:=\left(\begin{array}{cc}1 & j y \\ 0 & 1\end{array}\right)$ for a generator $y$ of $\mathbb{F}_{p^{n}}^{\times}$and some $j \in\{1, \ldots, p-1\}$.

Certainly, we get $\alpha(1)=1$ for any automorphism $\alpha \in \operatorname{Gal}\left(\mathbb{F}_{p^{n}} / \mathbb{F}_{p}\right)$. Let $\mathcal{S}$ be the set of subgroups of $\Gamma \mathrm{L}\left(p^{n}\right)$ which are generated by elements of $\operatorname{Gal}\left(\mathbb{F}_{p^{n}} / \mathbb{F}_{p}\right)$ and elements of a subgroup of $\Gamma \mathrm{L}_{0}\left(p^{n}\right)$ whose order is no multiple of

$$
\frac{p^{n}-1}{p-1} \cdot \prod_{\substack{p_{i} \in \mathbb{P} \\ p_{i} \mid d}} p_{i}^{r_{i}}
$$

Then, $\mathcal{S}$ does not contain a subgroup of $\Gamma \mathrm{L}\left(p^{n}\right)$ which acts transitively on the onedimensional subspaces of $\mathbb{F}_{p}^{n}$. However, the exponent of any subgroup of $\Gamma \mathrm{L}\left(p^{n}\right)$ not lying in $\mathcal{S}$ is divisible by

$$
\frac{p^{n}-1}{p-1} \cdot \prod_{\substack{p_{i} \in \mathbb{P} \\ p_{i} \mid d}} p_{i}^{r_{i}}
$$

whence the same holds for the exponent of $G$.

Remark 3.13. Analogously, one can show that the exponent of a subgroup of $\Gamma \mathrm{L}\left(p^{n}\right)$ which acts transitively on $\mathbb{F}_{p}^{n} \backslash\{0\}$ is a multiple of $p^{n}-1$.

The following lemma will help us to exclude the Suzuki 2-groups of type $A$ as Sylow 2-subgroups of a group whose character ring has finite representation type. 
Lemma 3.14. Let $P \in \operatorname{Syl}_{2}(G)$ be a Suzuki 2-group of type A. Then, the elements of order 4 of $G$ are nonreal.

Proof. Since Suzuki 2-groups are resistant, an element $x \in P$ of order 4 is conjugate to $x^{-1}$ in $G$ if and only if $x$ and $x^{-1}$ are conjugate in $N_{G}(P)$. Moreover, $\left|N_{G}(P): P\right|$ is odd. Therefore, $x$ and $x^{-1}$ are conjugate in $G$ if and only if there exists some $y \in P$ such that $y x y^{-1}=x^{-1}$. Recall that $P$ is isomorphic to

$$
A(n, \Theta):=\left\{\left(\begin{array}{ccc}
1 & a & b \\
0 & 1 & a^{\Theta} \\
0 & 0 & 1
\end{array}\right): a, b \in \mathbb{F}_{2^{n}}\right\}
$$

for a positive integer $n$ and some $1 \neq \Theta \in \operatorname{Aut}\left(\mathbb{F}_{2^{n}}\right)$ of odd order. Obviously,

$$
\left(\begin{array}{ccc}
1 & a & b \\
0 & 1 & a^{\Theta} \\
0 & 0 & 1
\end{array}\right)^{-1}=\left(\begin{array}{ccc}
1 & a & b+a^{1+\Theta} \\
0 & 1 & a^{\Theta} \\
0 & 0 & 1
\end{array}\right) .
$$

Let $A \in A(n, \Theta)$ be an element of order 4 . Then, we can find $a, b \in \mathbb{F}_{2^{n}}$ with $a \neq 0$ such that

$$
A=\left(\begin{array}{ccc}
1 & a & b \\
0 & 1 & a^{\Theta} \\
0 & 0 & 1
\end{array}\right) .
$$

If $A$ and $A^{-1}$ were conjugate in $A(n, \Theta)$, then there would exist $c, d \in \mathbb{F}_{2^{n}}$ with

$$
\begin{aligned}
\left(\begin{array}{ccc}
1 & a & b+a^{1+\Theta} \\
0 & 1 & a^{\Theta} \\
0 & 0 & 1
\end{array}\right) & =\left(\begin{array}{ccc}
1 & c & d \\
0 & 1 & c^{\Theta} \\
0 & 0 & 1
\end{array}\right)\left(\begin{array}{ccc}
1 & a & b \\
0 & 1 & a^{\Theta} \\
0 & 0 & 1
\end{array}\right)\left(\begin{array}{ccc}
1 & c & d+c^{1+\Theta} \\
0 & 1 & c^{\Theta} \\
0 & 0 & 1
\end{array}\right) \\
& =\left(\begin{array}{ccc}
1 & a+c & b+d+a^{\Theta} c \\
0 & 1 & a^{\Theta}+c^{\Theta} \\
0 & 0 & 1
\end{array}\right)\left(\begin{array}{ccc}
1 & c & d+c^{1+\Theta} \\
0 & 1 & c^{\Theta} \\
0 & 0 & 1
\end{array}\right) \\
& =\left(\begin{array}{ccc}
1 & a & d+c^{1+\Theta}+a c^{\Theta}+c^{1+\Theta}+b+d+a^{\Theta} c \\
0 & 1 & a^{\Theta} \\
0 & 0 & 1
\end{array}\right), \\
& =\left(\begin{array}{ccc}
1 & a & b+a c^{\Theta}+a^{\Theta} c \\
0 & 1 & a^{\Theta} \\
0 & 0 & 1
\end{array}\right)
\end{aligned}
$$

i.e. there would exist some $c \in \mathbb{F}_{2^{n}}$ with $a^{1+\Theta}=a^{\Theta} c+a c^{\Theta}$. In particular, $c \neq a$. Now,

$$
a^{1+\Theta}=a^{\Theta} c+a c^{\Theta} \quad \Leftrightarrow \quad 1=a^{-1} c+a^{-\Theta} c^{\Theta} \quad \Leftrightarrow \quad\left(a^{-1} c\right)^{\Theta}=1+a^{-1} c .
$$


This means that

$$
\left(a^{-1} c\right)^{\Theta^{2}}=\left(1+a^{-1} c\right)^{\Theta}=1+1+a^{-1} c=a^{-1} c
$$

but this yields a contradiction since $\Theta$ has odd order.

Lemma 3.15. Let $P \in \operatorname{Syl}_{2}(G)$ and suppose $R(G)$ has finite representation type. Then, $P$ is not isomorphic to a Suzuki 2-group of type A.

Proof. We assume that $P$ is a Suzuki 2-group of type $A$. Then, the elements of order 4 of $G$ are nonreal by Lemma 3.14. Since $Z(P)=P^{\prime}=\Omega_{1}(P)$, any linear character $\lambda \in \operatorname{Irr}(P)$ can only attain the values \pm 1 on the elements of order 4 of $P$. Moreover, $\chi(z)= \pm \chi(1)$ for $z \in Z(P)$ and $\chi \in \operatorname{Irr}(P)$. Since the degree of any nonlinear $\chi \in \operatorname{Irr}(P)$ is a 2-power, the values of $\chi$ lie in the maximal ideal of $\mathcal{O}_{\mathbb{Q}(i)}=\mathbb{Z}[i]$ which contains 2 , i.e. in $(1+i) \mathbb{Z}[i]$.

Let $x \in P$ be an element of order 4 . If $\chi$ is the character afforded by the nonlinear irreducible representation $\varrho$ of $P$, then the above yields that all eigenvalues of $\varrho\left(x^{2}\right)$ coincide and they are either 1 or -1 . Hence, all eigenvalues of $\varrho(x)$ are either contained in $\{1,-1\}$ or in $\{i,-i\}$. Thus, $\chi(x)$ is even contained in the ideal generated by 2 . This implies $\psi(x) \in \mathbb{Z}+2 \mathbb{Z}[i]$ for any nonlinear irreducible character $\psi \in \operatorname{Irr}(G)$. Additionally, there exists some $\varphi \in \operatorname{Irr}(G)$ with $\varphi(x) \notin \mathbb{R}$ because $x$ is nonreal. Hence, $\mathbb{Q}\left(\operatorname{cl}_{G}(x)\right)=\mathbb{Q}(i)$. We conclude that the function $\varphi_{x}$ with

$$
\varphi_{x}(g)= \begin{cases}1+i, & g \sim_{G} x \\ 1-i, & g \sim_{G} x^{-1} \\ 0, & \text { else }\end{cases}
$$

lies in $\operatorname{rad}\left(R(G)_{2}^{\prime}\right) \backslash R(G)_{2}$. Clearly, the function $\varphi_{1}$ with $\varphi_{1}(1)=2$ and $\varphi_{1}(g)=0$ else is also contained in $\operatorname{rad}\left(R(G)_{2}^{\prime}\right) \backslash R(G)_{2}$.

By our assumption, the $R(G)_{2}$-module $\operatorname{rad}\left(R(G)_{2}^{\prime} / R(G)_{2}\right)$ is cyclic. Thus, we find $\alpha \in \operatorname{rad}\left(R(G)_{2}^{\prime}\right)$ and $\eta_{1}, \eta_{2}, \xi_{1}, \xi_{2} \in R(G)_{2}$ with $\eta_{1} \alpha+\xi_{1}=\varphi_{1}$ and $\eta_{2} \alpha+\xi_{2}=\varphi_{x}$. Since $\alpha(x)$ and $\varphi_{x}(x)$ are contained in $(1+i) \mathbb{Z}[i]$, the same holds for $\xi_{2}(x)$. However, this implies $\xi_{2}(x) \in 2 \mathbb{Z}[i]$, whence $\alpha(x) \in(1+i) \mathbb{Z}[i] \backslash 2 \mathbb{Z}[i]$.

Moreover, we have $\left(\eta_{1} \alpha+\xi_{1}\right)(x)=\varphi_{1}(x)=0$. Similarly to the above, this yields $\xi_{1}(x) \in 2 \mathbb{Z}[i]$. Thus, $\eta_{1} \alpha(x) \in 2 \mathbb{Z}[i]$. Since $\alpha(x) \in(1+i) \mathbb{Z}[i] \backslash 2 \mathbb{Z}[i]$, this gives $\eta_{1}(x) \in(1+i) \mathbb{Z}[i]$, so $\eta_{1}(1) \in 2 \mathbb{Z}$. Certainly, $\alpha(1)$ lies also in $2 \mathbb{Z}$ and we conclude $\eta_{1} \alpha(1) \equiv 0(\bmod 4)$. Therefore, $\xi_{1}(1) \equiv 2(\bmod 4)$.

Since $x^{2} \in Z(P) \cap P^{\prime}$, we get $\psi(1) \equiv \psi\left(x^{2}\right)(\bmod 4)$ for any $\psi \in \operatorname{Irr}(P)$. That is why $\chi(1) \equiv \chi\left(x^{2}\right)(\bmod 4)$ for any $\chi \in \operatorname{Irr}(G)$ because $\chi_{P}$ is a $\mathbb{Z}$-linear combination of irreducible characters of $P$. This implies $\xi_{1}\left(x^{2}\right) \equiv \xi_{1}(1) \equiv 2(\bmod 4)$. 
On the other hand, $\eta_{1} \alpha\left(x^{2}\right)$ is divisible by 4 since $\eta_{1}\left(x^{2}\right)$ is even and $\alpha$ is contained in $\operatorname{rad}\left(R(G)_{2}^{\prime}\right)$. But this yields $\left(\eta_{1} \alpha+\xi_{1}\right)\left(x^{2}\right) \equiv 2 \not \equiv 0 \equiv \varphi_{1}\left(x^{2}\right)(\bmod 4)$, so $P$ cannot be a Suzuki 2-group of type $A$.

However, the remaining Suzuki 2-groups can also not occur as Sylow 2-subgroups of $G$ if $R(G)$ is representation-finite.

Lemma 3.16. Let $P \in \operatorname{Syl}_{2}(G)$ and suppose $R(G)$ has finite representation type. Then, $P$ is not isomorphic to a Suzuki 2-group of type B, C, or D.

Proof. We assume that $P$ is a Suzuki 2-group of type $B, C$, or $D$, so $|P|=|Z(P)|^{3}$. Since $R(G)$ is representation-finite, all elements of order 4 of $G$ are conjugate in $G$. As mentioned above, Suzuki 2-groups are resistant, whence there is only one conjugacy class of elements of order 4 in $N_{G}(P)$.

Let $x \in P$ be an element of order 4 . It suffices to show $\chi(1) \equiv \chi\left(x^{2}\right) \equiv \chi(x)$ $(\bmod 4)$ for all $\chi \in \operatorname{Irr}(G)$. Then, it can be easily seen that for any $\alpha \in R(G)_{2}^{\prime}$, there do not exist $\eta_{1}, \eta_{2}, \xi_{1}, \xi_{2} \in R(G)_{2}$ such that the equations

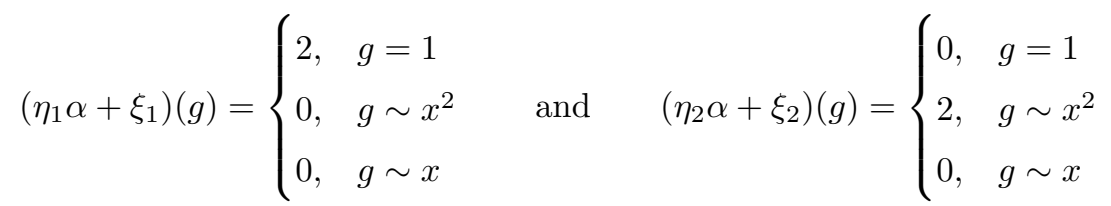

hold simultaneously. Thus, it already suffices to show $\psi(1) \equiv \psi\left(x^{2}\right) \equiv \psi(x)$ $(\bmod 4)$ for all $\psi \in \operatorname{Irr}\left(N_{G}(P)\right)$. It is clear that for $\vartheta \in \operatorname{Irr}(P), \vartheta(1) \equiv \vartheta\left(x^{2}\right)$ $(\bmod 4)$ because $Z(P)=P^{\prime}$ and $P$ is a 2-group. Therefore, we only have to consider the elements of order 4 .

We choose $n$ such that $|Z(P)|=2^{n}$. Then, $P / P^{\prime} \cong C_{2}^{2 n}$ whence $\mid\{\lambda \in \mathrm{P}$ : $\lambda(1)=1\} \mid=2^{2 n}$. This gives $\left|C_{P}(x)\right| \geq 2^{2 n}$. Let $j \in \mathbb{N}$ be such that $\left|C_{P}(x)\right|=2^{j}$, so $\left|\mathrm{cl}_{P}(x)\right|=2^{3 n-j}$ with $j \geq 2 n$. Now,

$$
2^{3 n}=|P|=|Z(P)|+|P \backslash Z(P)|=2^{n}+2^{3 n-j}\left(2^{j}-2^{j-2 n}\right) .
$$

This shows that $P$ contains exactly $2^{j}-2^{j-2 n}=2^{j-2 n}\left(2^{2 n}-1\right)$ conjugacy classes of elements of order 4. Since $N_{G}(P) / C_{G}(P)$ is a group of odd order which acts transitively on these classes, we conclude $j=2 n$, i.e. $\left|C_{P}(x)\right|=2^{2 n}$. Thus, for any nonlinear character $\vartheta \in \operatorname{Irr}(P)$, we get $\vartheta(x)=0$. Hence, any irreducible character of $P / Z(P)$ has the form $\hat{\lambda}$ with $\hat{\lambda}(y Z(P)):=\lambda(y)$ for all $y \in P$ where $\lambda$ is a linear character of $P$, and if $\lambda_{1}, \lambda_{2}$ are linear characters of $P$ with $\lambda_{1} \neq \lambda_{2}$, then $\hat{\lambda}_{1}, \hat{\lambda}_{2}$ are irreducible characters of $P / Z(P)$ with $\hat{\lambda}_{1} \neq \hat{\lambda}_{2}$.

Certainly, $N_{G}(P) / C_{G}(P)$ acts transitively on the nontrivial elements of $P / Z(P)$. Thus, $N_{G}(P) / C_{G}(P)$ is a subgroup of $\Gamma \mathrm{L}\left(2^{2 n}\right)$ by Theorem 3.11. Furthermore, $\exp \left(N_{G}(P) / C_{G}(P)\right)$ is divisible by $2^{2 n}-1$. Consequently, $P / Z(P) \rtimes N_{G}(P) / C_{G}(P)$ 
contains a subgroup isomorphic to $A_{4}$. But if $t \in A_{4}$ is an involution, then we have $\varphi(1) \equiv \varphi(t)(\bmod 4)$ for any $\varphi \in \operatorname{Irr}\left(A_{4}\right)$. This yields $\varphi(Z(P)) \equiv \varphi(x Z(P))$ $(\bmod 4)$ for all $\varphi \in \operatorname{Irr}\left(P / Z(P) \rtimes N_{G}(P) / C_{G}(P)\right)$.

Finally, the action of an element $h \in N_{G}(P) / C_{G}(P)$ on a character $\hat{\lambda} \in \operatorname{Irr}(P / Z(P))$ corresponds to the action of a preimage $\bar{h} \in N_{G}(P)$ of $h$ on $\lambda$ because

$$
\hat{\lambda}^{h}(y Z(P))=\hat{\lambda}\left(h y Z(P) h^{-1}\right)=\lambda\left(\bar{h} y \bar{h}^{-1}\right)=\lambda^{\bar{h}}(y) .
$$

This implies $\psi(1) \equiv \psi(x)(\bmod 4)$ for all $\psi \in N_{G}(P)$ and we are done.

With these preparations, we are able to prove our main theorem of this section.

Theorem 3.17. Let $G$ be a group of even order whose character ring has finite representation type. Then, every Sylow 2-subgroup of $G$ is isomorphic to one of the following groups:

(1) $C_{2}^{n}$ for some $n \geq 1$ such that $2^{n}-1$ is cube-free,

(2) $\mathrm{C}_{4}$,

(3) $Q_{8}$,

(4) $D_{8}$.

Proof. Let $P \in \operatorname{Syl}_{2}(G)$. If $E(G) \neq 1$, then $E(G)$ is a Hall subgroup of $G$ by Theorem 3.8. Therefore, $P$ is a Sylow 2-subgroup of $E(G)$ and we can write $G=$ $E(G) \rtimes H$ for a suitable subgroup $H$. By Proposition 3.5, $G$ contains only one nonabelian composition factor, so Theorem 3.8 yields that $P$ is either elementaryabelian or isomorphic to $Q_{8}$ or $D_{8}$.

From now on, we do not suppose that $R(G)$ is representation-finite, but make the following weaker assumptions:

(1) $G$ has at most three $\mathbb{Q}$-classes of 2-elements,

(2) $P$ is not isomorphic to a Suzuki 2-group of type $A$,

(3) if $E(G) \neq 1$, then $P$ is isomorphic to one of the groups in the list above.

Suppose $G$ is not solvable and $E(G)=1$. The Sylow 2-subgroups of $G / O_{2^{\prime}}(G)$ are isomorphic to $P$ and by Lemma 3.1, $G / O_{2^{\prime}}(G)$ contains at most as many $\mathbb{Q}$-classes of 2 -elements as $G$ does. Thus, we may assume $O_{2^{\prime}}(G)=1$, so either $E(G) \neq 1$ or $F^{*}(G)=O_{2}(G)$. Since the case $E(G) \neq 1$ has already been discussed, we suppose $F^{*}(G)=\mathrm{O}_{2}(G)$.

Since $G$ is not solvable, $O_{2}(G)$ is a proper subgroup of $P$. The elements of $P \backslash O_{2}(G)$ are certainly not conjugate to the nontrivial elements of $O_{2}(G)$. Thus, there is only one $\mathbb{Q}$-class in $G$ which contains nontrivial elements of $O_{2}(G)$, i.e. the nontrivial elements of $\mathrm{O}_{2}(G)$ form a single conjugacy class. Hence, $G$ acts 
transitively on $O_{2}(G) \backslash\{1\}$. Moreover, $C_{G}\left(O_{2}(G)\right)=O_{2}(G)$, so $G / O_{2}(G)$ is isomorphic to a transitive linear group. Additionally, there is only one conjugacy class of nontrivial 2-elements in $\mathrm{G} / \mathrm{O}_{2}(G)$.

By Theorem 3.11, the only possibility is that $G / O_{2}(G)$ contains a normal subgroup isomorphic to $\mathrm{SL}\left(2,2^{m}\right)$ where $m$ satisfies $O_{2}(G) \cong C_{2}^{2 m}$. But then, there exists an involution in $G \backslash O_{2}(G)$ by [10, Lemma 4.2]. Lemma 3.3 then implies that $R(G)$ has infinite representation type. Thus, $F^{*}(G) \neq O_{2}(G)$ if $G$ is not solvable.

Now, suppose $G$ is solvable. If $P$ has only one involution, then, due to Burnside, $P$ is isomorphic to a cyclic group or a generalized quaternion group. All of these groups except $C_{2}, C_{4}$ and $Q_{8}$ have exponents divisible by 8 , so $P$ is isomorphic to $C_{2}, C_{4}$ or $Q_{8}$.

Finally, we assume that $P$ has more than one involution. By Thompson, $P$ is homocyclic or isomorphic to a Suzuki 2-group (see [27]). By the Lemmas 3.15 and 3.16, $P$ cannot be a Suzuki 2 -group. This implies that $P$ is homocyclic, whence $P$ is elementary-abelian by Theorem 3.2 . Thus, there is some $n$ such that $P \cong$ $C_{2}^{n}$. We have already seen in Lemma 3.3 that all involutions of $P$ are conjugate. Therefore, $G / C_{G}(P)$ acts transitively on $P \backslash\{1\}$. Now, Theorem 3.11 yields that $G / C_{G}(P)$ is a subgroup of $\Gamma \mathrm{L}\left(2^{n}\right)$, so $\exp \left(G / C_{G}(P)\right)$ is a multiple of $2^{n}-1$ by Lemma 3.12. It follows immediately that $2^{n}-1$ is cube-free.

For each 2-group $P$ in the list of Theorem 3.17, there is a group $G$ such that $P$ is a Sylow 2-subgroup of $G$ and $R(G)$ is representation-finite. For $P \in\left\{C_{2}, C_{4}\right\}$, we can choose $G=P$, for $P=Q_{8}$, we can take $G=\mathrm{SL}(2,3)$, and if $P=D_{8}$, then the conditions are satisfied for $G \cong \operatorname{PSL}(2,7)$. Moreover, for $P=C_{2}^{n}$ and $G=C_{2}^{n} \rtimes C_{2^{n}-1}$, the representation type of $R(G)$ is finite if and only if $2^{n}-1$ is cube-free.

3.3. Sylow subgroups of odd order. After the determination of the possible Sylow 2-subgroups of a group $G$ whose character ring has finite representation type, we consider the Sylow subgroups of odd order of $G$. Finally, we will prove that these Sylow subgroups are abelian in Theorem 3.20.

Lemma 3.18. Let $p$ be an odd prime, $P \in \operatorname{Syl}_{p}(G)$ be nonabelian, and $R(G)$ be representation-finite. If $G$ has exactly three $\mathbb{Q}$-classes of $p$-elements, then all nontrivial elements of $Z(P)$ are conjugate in $G$.

Proof. At first, we show that all elements of $Z(P) \backslash\{1\}$ are contained in one of the two $\mathbb{Q}$-classes which consist of nontrivial $p$-elements.

If $G$ has a component whose order is divisible by $p$, then $E(G)$ contains a Sylow $p$-subgroup of $G$ by Theorem 3.8. However, Corollary 3.9 then yields that $P$ is abelian. Thus, $p \nmid|E(G)|$. That is why $G / E(G)$ has Sylow $p$-subgroups isomorphic 
to $P$ and by Lemma $3.1, G / E(G)$ has at most as many $\mathbb{Q}$-classes of $p$-elements as $G$ has. Moreover, for $x \in G$, the conjugacy class of $x E(G)$ in $G / E(G)$ is rational if the conjugacy class of $x$ in $G$ is rational.

That is why we may assume $E(G)=1$ in the following. Since the Sylow $p$ subgroups of $G / O_{p^{\prime}}(G)$ are isomorphic to $P$, we further assume $O_{p^{\prime}}(G)=\{1\}$. Lemma 3.1 again yields that $G / O_{p^{\prime}}(G)$ contains at most as many $\mathbb{Q}$-classes of $p$ elements as $G$ does and that the image of a rational conjugacy class of $G$ is a rational conjugacy class of $G / O_{p^{\prime}}(G)$.

Hence, we suppose $F^{*}(G)=O_{p}(G)$, so $C_{G}\left(O_{p}(G)\right) \leq O_{p}(G)$. If $O_{p}(G)<P$, this implies $Z(P) \leq O_{p}(G)$. Since the elements of $P \backslash O_{p}(G)$ are not conjugate to the elements of $O_{p}(G)$ in $G, G$ has two $\mathbb{Q}$-classes of nontrivial $p$-elements and only one of these classes contains elements of $O_{p}(G) \geq Z(P)$. On the other hand, if $O_{p}(G)=P$, then $P$ is normal in $G$, whence $Z(P)$ is normal in $G$. Since $P$ is nonabelian, $P \backslash Z(P) \neq \emptyset$ and none of the elements of $P \backslash Z(P)$ is $\mathbb{Q}$-conjugate to an element of $Z(P)$.

Thus, it remains to show that the $\mathbb{Q}$-class of $G$ which contains the nontrivial elements of $Z(P)$ is a rational conjugacy class. As is well known, there exists a nontrivial element $z \in Z(P) \cap P^{\prime}$. We show that $z$ lies in a rational conjugacy class of $G$.

Suppose the conjugacy class of $z$ in $G$ is not rational. We denote the maximal ideal of $\mathcal{O}_{\mathbb{Q}\left(\operatorname{cl}_{G}(z)\right)}$ containing $p$ by $\mathfrak{p}$.

For any linear character $\lambda \in \operatorname{Irr}(P)$, we have $\lambda(z)=1$ because $z \in P^{\prime}$. Moreover, for every $\psi \in \operatorname{Irr}(P)$, there is some $k \in \mathbb{Z}$ such that $\psi(z)=\psi(1) \cdot \zeta_{p}^{k}$ since $z \in Z(P)$. Certainly, $\psi(1)$ is a power of $p$. The restriction of $\chi \in \operatorname{Irr}(G)$ to $P$ can be written as $\chi_{P}=a_{1} \psi_{1}+\ldots+a_{r} \psi_{r}$ for suitable $a_{1}, \ldots, a_{r} \in \mathbb{Z}$ and $\psi_{1}, \ldots, \psi_{r} \in \operatorname{Irr}(P)$. Let $\psi_{1}, \ldots, \psi_{m}$ denote the nonlinear characters of this linear combination, and $\psi_{m+1}, \ldots, \psi_{r}$ denote the linear characters. For $j=1, \ldots, m$, choose $k_{j} \in\{0, \ldots, p-$ $1\}$ such that $\psi_{j}(z)=\psi_{j}(1) \cdot \zeta_{p}^{k_{j}}$, and $d_{j} \in \mathbb{N}$ such that $\psi_{j}(1)=d_{j} \cdot p$. Then,

$$
\begin{aligned}
\chi(1)-\chi(z) & =\sum_{j=1}^{m} a_{j}\left(\psi_{j}(1)-\psi_{j}(z)\right)+\sum_{j=m+1}^{r} a_{j}\left(\psi_{j}(1)-\psi_{j}(z)\right) \\
& =\sum_{j=1}^{m} a_{j}\left(p d_{j}-p d_{j} \zeta_{p}^{k_{j}}\right)=\sum_{j=1}^{m} a_{j} d_{j} p\left(1-\zeta_{p}^{k_{j}}\right) .
\end{aligned}
$$

Let $\mathfrak{P}$ be the maximal ideal in $\mathbb{Z}\left[\zeta_{p}\right]$ containing $p$. Then, each summand of the sum above lies in $p \mathfrak{P}$. Hence, $\chi(1)-\chi(z) \in p \mathfrak{p}$. Since $\left[\mathbb{Q}\left(\operatorname{cl}_{G}(z)\right): \mathbb{Q}\right]>1$, the ideal $p \mathcal{O}_{\mathbb{Q}\left(\operatorname{cl}_{G}(z)\right)}$ is contained in $\mathfrak{p}^{2}$. This implies $\chi(1) \equiv \chi(z)\left(\bmod \mathfrak{p}^{3}\right)$. Therefore, any function $\eta \in R(G)_{p}$ with $\eta(z) \in \mathfrak{p}$ satisfies $\eta(1) \equiv \eta(z)\left(\bmod \mathfrak{p}^{3}\right)$. That is why for 
a generator $\pi$ of $\mathfrak{p}$, the functions $\varphi_{1}, \varphi_{2} \in R(G)_{p}^{\prime}$ with

$$
\varphi_{1}(g)=\left\{\begin{array}{ll}
\pi, & g \sim z \\
0, & \text { else }
\end{array} \quad \text { and } \quad \varphi_{2}(g)= \begin{cases}\pi^{2}, & g \sim z \\
0, & \text { else }\end{cases}\right.
$$

lie in $\operatorname{rad}\left(R(G)_{p}^{\prime}\right) \backslash R(G)_{p}$.

Let $\alpha \in \operatorname{rad}\left(R(G)_{p}^{\prime}\right)$ and $\eta_{1}, \eta_{2}, \xi_{1}, \xi_{2} \in R(G)_{p}$ with $\eta_{1} \alpha+\xi_{1}=\varphi_{1}$ and $\eta_{2} \alpha+\xi_{2}=$ $\varphi_{2}$. Since $\alpha(z) \in \mathfrak{p}$, we conclude $\xi_{1}(z) \in \mathfrak{p}$. But then, $\xi_{1}(z)$ is even contained in $\mathfrak{p}^{3}$, so $\alpha(z) \in \mathfrak{p} \backslash \mathfrak{p}^{2}$.

Analogously, $\xi_{2}(z) \in \mathfrak{p}$, so $\xi_{2}(z) \in \mathfrak{p}^{3}$. Thus, $\eta_{2} \alpha(z) \in \mathfrak{p}^{2} \backslash \mathfrak{p}^{3}$. But this yields $\eta_{2}(z) \in \mathfrak{p} \backslash \mathfrak{p}^{2}$ which is impossible since $\eta_{2} \in R(G)_{p}$. Hence, the $R(G)_{p}$-module $\operatorname{rad}\left(R(G)_{p}^{\prime} / R(G)_{p}\right)$ is not cyclic, so $R(G)$ has infinite representation type. This contradiction shows that the $\mathbb{Q}$-class of $z$ is already a conjugacy class.

In the proof of Lemma 3.18, we have shown implicitly that $G$ contains at least (and therefore exactly) three $\mathbb{Q}$-classes of $p$-elements if $P$ is nonabelian and $R(G)$ is representation-finite. Thus, we could remove the assumption in Lemma 3.18 that $G$ has exactly three $\mathbb{Q}$-classes of $p$-elements.

Before we prove our main theorem of this section, we remark that the group $\mathrm{SL}(2,5)$ can only act trivially on a cyclic group since the automorphism group of a cyclic group is solvable while $\mathrm{SL}(2,5)$ is nonsolvable and perfect. Moreover, we recall a result due to Shult [38].

Theorem 3.19 (Shult). Let $P$ be a p-group such that $\operatorname{Aut}(P)$ acts transitively on the subgroups of order $p$ of $P$. Then, $P$ is abelian or a 2 -group.

Theorem 3.20. Let $p$ be an odd prime, and $P \in \operatorname{Syl}_{p}(G)$. If $R(G)$ has finite representation type, then $P$ is abelian.

Proof. Suppose $P$ is nonabelian and $R(G)$ has finite representation type. As in the proof of Lemma 3.18, we conclude $p \nmid|E(G)|$. That is why we only consider the case $p \nmid|E(G)|$ in the following.

From now on, we do not assume that $R(G)$ is representation-finite, but make the following weaker assumptions:

(1) $G$ has at most three $\mathbb{Q}$-classes of $\ell$-elements for any prime $\ell$,

(2) all elements of $Z(P)$ are conjugate in $G$,

(3) the Sylow 2-subgroups of $G$ appear in the list of Theorem 3.17.

Let $P$ be nonabelian. If $E(G) \neq 1$, then the Sylow $p$-subgroups of $G / E(G)$ are isomorphic to $P$ and $G / E(G)$ has at most as many $\mathbb{Q}$-classes of $p$-elements as $G$ has. Moreover, for any $p$-element $x \in G$, the conjugacy class of $x E(G)$ in $G / E(G)$ is rational if the conjugacy class of $x$ in $G$ is rational. 
We can obtain similar assertions for $G / O_{p^{\prime}}(G)$. That is why we assume $E(G)=1$ and $O_{p^{\prime}}(G)=1$ in the following. This yields $F^{*}(G)=O_{p}(G)$, so $C_{G}\left(O_{p}(G)\right) \leq$ $O_{p}(G)$.

At first, we consider the case $O_{p}(G)<P$. Since $C_{G}\left(O_{p}(G)\right) \leq O_{p}(G)$, we get $Z(P) \leq O_{p}(G)$. It is clear that none of the elements of $P \backslash O_{p}(G)$ is conjugate to an element of $O_{p}(G)$. Thus, there is only one $\mathbb{Q}$-class in $G$ containing elements of $P \backslash O_{p}(G)$. Similarly, all nontrivial elements of $O_{p}(G)$ are $\mathbb{Q}$-conjugate. Moreover, the nontrivial elements of $Z(G)$ are conjugate in $G$, so $G$ acts transitively on $O_{p}(G) \backslash$ $\{1\}$. Together with Theorem 3.19, this implies that $O_{p}(G)$ is elementary-abelian and $G / O_{p}(G)$ is isomorphic to one of the groups in the list of Theorem 3.11. Prior to going through this list, we make some more general considerations.

(1) Let $m$ be a positive integer such that $O_{p}(G) \cong C_{p}^{m}$. Then, the number of $p$ elements in $G \backslash O_{p}(G)$ is divisible by $p^{m}$. Suppose the Sylow $p$-subgroups of $G / O_{p}(G)$ are cyclic and $G \backslash O_{p}(G)$ contains an element of order $p$. For any $x \in P \cap\left(G \backslash O_{p}(G)\right), p^{2}$ divides $\left|C_{G}(x)\right|$ since $Z(P) \leq O_{p}(G)$. Therefore,

$$
\left|\mathrm{cl}_{G}(x)\right|=\left|G: C_{G}(x)\right| \not \equiv 0 \quad\left(\bmod p^{m}\right) .
$$

The $\mathbb{Q}$-class of $x$ in $G$ contains exactly $r \cdot\left|\mathrm{cl}_{G}(x)\right|$ elements for a suitable $r \in\{1, \ldots, p-1\}$. This implies that $p^{m}$ does not divide the number of elements in the $\mathbb{Q}$-class of $x$. Hence, the elements of order $p$ lie in at least two different $\mathbb{Q}$-classes. But then, $G$ would have at least four $\mathbb{Q}$-classes of $p$-elements.

(2) Suppose $O_{p}(G) \cong C_{p}^{2}$ and the Sylow $p$-subgroups of $G / O_{p}(G)$ are elementary-abelian of rank $\ell>1$. Let $Q=P / O_{p}(G) \in \operatorname{Syl}_{p}\left(G / O_{p}(G)\right)$. We choose generators $x_{1}, \ldots, x_{\ell}$ of $Q$. Additionally, we denote the cyclic subgroups of order $p$ of $O_{p}(G)$ by $Z_{1}, \ldots, Z_{p+1}$. Since $Z(P) \leq O_{p}(G), Q$ acts trivially on one of these cyclic groups, say $Z_{p+1}$. On the other hand, none of the nontrivial elements of $Q$ can act trivially on $O_{p}(G)$ because $C_{G}\left(O_{p}(G)\right) \leq O_{p}(G)$. Thus, any nontrivial element of $Q$ induces a cyclic permutation of $Z_{1}, \ldots, Z_{p}$. W.l.o.g. we have $x_{1} Z_{1} x_{1}^{-1}=Z_{2}$. Since $x_{2}$ also permutes $Z_{1}, \ldots, Z_{p}$ cyclically, we can find some $j \in\{1, \ldots, p-1\}$ such that $x_{2}^{j} Z_{2} x_{2}^{-j}=Z_{1}$. But then, $x_{2}^{j} x_{1}$ centralizes $Z_{p+1}$ and $Z_{1}$, i.e. $x_{2}^{j} x_{1}$ acts trivially on $O_{p}(G)$. This contradicts $C_{G}\left(O_{p}(G)\right) \leq O_{p}(G)$.

(3) Let $O_{p}(G) \cong C_{p}^{m}$ again. Suppose that each element of order $p$ of $O_{p}(G)$ has a centralizer of odd order in $G$, and there exists an element of order $p$ in $G / O_{p}(G)$ which commutes with an involution of $G / O_{p}(G)$. Then, there is an element $\bar{x}$ of order $2 p$ in $G / O_{p}(G)$. Thus, a preimage $x$ of $\bar{x}$ in $G$ has order $2 p$ or $2 p^{2}$. If $|\langle x\rangle|=2 p^{2}$, then any element of $G \backslash O_{p}(G)$ would have 
order $p^{2}$, whence $x^{2 p} \in O_{p}(G)$. But then, $\left|C_{G}\left(x^{2 p}\right)\right|$ would be odd whereas $x$ has even order. This implies that $G \backslash O_{p}(G)$ contains elements of order $p$.

Now, we go through the list of Theorem 3.11. Let $O_{p}(G) \cong C_{p}^{m}$. We assume that $G / O_{p}(G)$ acts on $O_{p}(G)$ as a subgroup of $\Gamma \mathrm{L}\left(p^{m}\right) \cong C_{p^{m}-1} \rtimes C_{m}$ on $\mathbb{F}_{p}^{m}$, so $G / O_{p}(G) \cong C_{r} \rtimes C_{s}$ with $r \mid p^{m}-1$ and $s \mid m$. Since this action is transitive, $G / O_{p}(G)$ contains an involution. Moreover, $p$ divides $\left|G / O_{p}(G)\right|$, whence $p \mid s$. If $\left|C_{r}\right|$ is even, then $\left|Z\left(G / O_{p}(G)\right)\right|$ is clearly even as well. On the other hand, if $\left|C_{s}\right|$ is even, then there exist $x, y \in C_{s}$ such that $|\langle x\rangle|=p,|\langle y\rangle|=2$ and $x y=y x$. In both cases, $G \backslash O_{p}(G)$ contains elements of order $p$ by (3). Obviously, the Sylow $p$-subgroups of $G / O_{p}(G)$ are cyclic, so $G$ possesses at least four $\mathbb{Q}$-classes of $p$-elements by (1).

Let $k \geq 2$ and suppose $G / O_{p}(G)$ contains a normal subgroup isomorphic to $\operatorname{SL}(k, q)$. Then, $k=2$ and $q$ is a prime number by Theorem 3.8, so $m=2$. Due to (2), $G / O_{p}(G)$ has cyclic Sylow $p$-subgroups. Since $G / O_{p}(G)$ has a subgroup isomorphic to $\operatorname{SL}(2, p)$, each element of order $p$ of $G / O_{p}(G)$ has a centralizer of even order. We conclude that $G \backslash O_{p}(G)$ contains elements of order $p$ by (3). But now, (1) yields that $G$ has at least four $\mathbb{Q}$-classes of $p$-elements.

In the case that $G / O_{p}(G)$ has a normal subgroup isomorphic to $\operatorname{Sp}(k, q)$ (with $p^{m}=q^{k}, k \in 2 \mathbb{N}$ ), we also obtain $m=2$. Thus, the same arguments as above show that this situation cannot arise as well.

Suppose $p=3, O_{3}(G) \cong C_{3}^{2}$, and $G / O_{3}(G)$ has a normal subgroup isomorphic to $Q_{8}$. Then, $Z\left(G / O_{3}(G)\right)$ contains an element of order 2. By (3) and (1), the Sylow 3subgroups of $G / O_{3}(G)$ cannot be cyclic. Moreover, they are not elementary-abelian of rank $>1$ by (2), so this case can also be excluded.

Suppose $p \in\{5,7,11,23\}, O_{p}(G) \cong C_{p^{2}}$, and $G / O_{p}(G)$ contains a normal subgroup $H \cong \mathrm{SL}(2,3)$. Then, $G / O_{p}(G)$ has cyclic Sylow $p$-subgroups by (2). Certainly, $Z\left(G / O_{p}(G)\right)$ contains the involution in $H$. Now, (3) and (1) yield that $G$ has at least four $\mathbb{Q}$-classes of $p$-elements.

We can argue similarly in the case that $p \in\{11,19,29,59\}, O_{p}(G) \cong C_{p^{2}}$, and $G / O_{p}(G)$ contains a normal subgroup isomorphic to $\mathrm{SL}(2,5)$. From (2), we obtain that $G / O_{p}(G)$ has cyclic Sylow $p$-subgroups. Since an element of order $p$ can only act trivially on $\mathrm{SL}(2,5), G / O_{p}(G)$ has elements of order $2 p$. Applying (3) and (1), this shows that there are more than three $\mathbb{Q}$-classes of $p$-elements in $G$.

Suppose $p=3$ and $O_{3}(G) \cong C_{3}^{4}$. By Theorem 3.17, $G / O_{3}(G)$ has no normal Sylow 2-subgroup which is an extraspecial group of order 32. Thus, $G / O_{3}(G)$ has a normal subgroup $H \cong \mathrm{SL}(2,5)$. The action of $H$ on $O_{3}(G) \backslash\{1\}$ is not transitive; $O_{3}(G) \backslash\{1\}$ splits into two orbits under this action. Hence, $G / O_{3}(G)$ can only act 
transitively on $O_{3}(G) \backslash\{1\}$ if $\left(G / O_{3}(G)\right) / H$ has even order. But then, $G / O_{3}(G)$ would have at least four $\mathbb{Q}$-classes of 2-elements.

Finally, if $p=3, O_{3}(G) \cong C_{3}^{6}$, and $G / O_{p}(G) \cong \mathrm{SL}(2,13)$, we conclude that $G$ would have at least four $\mathbb{Q}$-classes of 3 -elements by (3) and (1) as above.

That is why we may assume $P \unlhd G$. Recall that all nontrivial elements of $Z(P)$ are conjugate and all elements of $P \backslash Z(P)$ lie in the same $\mathbb{Q}$-class of $G$. By Theorem 3.19, $Z(P)$ as well as $P / Z(P)$ are elementary-abelian and we have $\exp (P)=p$. Since all nontrivial elements of $Z(P)$ are conjugate in $G$, there is no subgroup $1<H<Z(P)$ which is normal in $G$. This yields $Z(P) \leq P^{\prime}$. On the other hand, $P / Z(P)$ is abelian, whence $\Phi(P) \leq Z(P)$. Due to this and $\exp (P)=p$, we get $P^{\prime}=\Phi(P)=Z(P)$, i.e. $P$ is special.

Let $Z(P) \cong C_{p}^{k}$ and $P / Z(P) \cong C_{p}^{\ell}$. Since all elements of $P \backslash Z(P)$ lie in the same $\mathbb{Q}$-class of $G$, we get $\left|C_{P}(x)\right|=\left|C_{P}(y)\right|$ for $x, y \in P \backslash Z(P)$. We choose $j$ such that $\left|C_{P}(x)\right|=p^{j}$. Thus, the conjugacy classes of $P$ which contain elements of $P \backslash Z(P)$ have length $p^{k+\ell-j}$. Now,

$$
p^{k+\ell}=1 \cdot p^{k}+p^{k+\ell-j}\left(p^{j}-p^{j-\ell}\right),
$$

so there are exactly $p^{j}-p^{j-\ell}=p^{j-\ell}\left(p^{\ell}-1\right)$ conjugacy classes in $P$ containing elements of $P \backslash Z(P)$. That is why the number of these conjugacy classes is not divisible by $p$ if and only if $j=\ell$. Clearly, the same holds for the number of the corresponding $\mathbb{Q}$-classes of $P$. It is necessary that $p$ does not divide the number of these $\mathbb{Q}$-classes: $G$ permutes these classes transitively. If $\mathcal{M}$ is the set of these classes, there is a bijection between $\mathcal{M}$ and $G / \operatorname{stab}_{G}(\mathcal{C})$ for some $\mathcal{C} \in \mathcal{M}$. Since $P$ lies in $\operatorname{stab}_{G}(\mathcal{C}), p$ cannot divide $\left|G: \operatorname{stab}_{G}(\mathcal{C})\right|=|\mathcal{M}|$. This gives $\left|C_{P}(x)\right|=p^{\ell}$ for $x \in P \backslash Z(P)$, whence

$$
|\{[x, y]: y \in P\}|=\frac{p^{k+\ell}}{\left|C_{P}(x)\right|}=\frac{p^{k+\ell}}{p^{\ell}}=p^{k}=|Z(P)| .
$$

Since $P^{\prime}=Z(P)$, this means that for all $z \in Z(P)$, there exists some $y \in P$ with $z=[x, y]$.

Let $H$ be a maximal subgroup of $Z(P)$. Certainly, we have $\exp (P / H)=p$. For $z \in Z(P) \backslash H$, we have just seen that there are $x, y \in P$ with $z=[x, y]$. This yields $z H \in(P / H)^{\prime}$, so $(P / H)^{\prime}=Z(P) / H$ is cyclic. Moreover, for every $x \in P \backslash Z(P)$, there exists some $y \in P$ such that $[x, y] \in Z(P) \backslash H$. Hence, $x H \notin Z(P / H)$ and we conclude $Z(P / H)=Z(P) / H$, i.e. $Z(P / H)=(P / H)^{\prime} \cong C_{p}$. Consequently, the group $P / H$ is extraspecial. From the classification of extraspecial $p$-groups, we obtain at once that $\ell$ is even.

Now, $P / Z(P)$ is an elementary-abelian group of even rank whose cyclic subgroups are permuted transitively by $G / Z(P)$. By Theorem 3.11 and since $G$ has at 
most three $\mathbb{Q}$-classes of $p$-elements, $G / C_{G}(P)$ acts on $P / Z(P)$ either as a subgroup of $\Gamma L\left(p^{\ell}\right)$ on $\mathbb{F}_{p}^{\ell}$ or as one of the sporadic transitive linear groups if $|P / Z(P)|=3^{4}$ or $p \in\{3,5,7,11,19,23,29,59\}$ where $P$ is an extraspecial group of order $p^{3}$ with exponent $p$.

At first, we assume that $G / C_{G}(P)$ acts on $P / Z(P)$ as a subgroup of $\Gamma \mathrm{L}\left(p^{\ell}\right)$ on $\mathbb{F}_{p}^{\ell}$. By Lemma 3.12, the exponent of $G / C_{G}(P)$ is divisible by

$$
\frac{p^{\ell}-1}{p-1} \cdot \prod_{\substack{p_{i} \in \mathbb{P} \\ p_{i} \mid d}} p_{i}^{r_{i}}
$$

where $d:=\operatorname{gcd}\left(\left(p^{n}-1\right) /(p-1), p-1\right)$ and the $r_{i}$ are the largest integers such that $p_{i}^{r_{i}}$ divides $p-1$. However, $\ell$ is even, so $\left(p^{\ell}-1\right) /(p-1)$ is also even. Thus, $\exp \left(G / C_{G}(P)\right)$ is divisible by 8 , i.e. $G / C_{G}(P)$ has an element of order 8 . This is clearly impossible.

Hence, $|P / Z(P)|=3^{4}$ or $P$ is extraspecial of order $p^{3}$ with exponent $p$ where $p \in\{3,5,7,11,19,23,29,59\}$.

(1) If $|P / Z(P)|=3^{2}$, then $G / C_{G}(P)$ has a normal subgroup isomorphic to $Q_{8}$. Since all elements of order 4 of $G$ are conjugate, $G \backslash P$ must contain an element of order 3. But then, $G$ has at least four $\mathbb{Q}$-classes of 3-elements.

(2) Suppose $|P / Z(P)|=3^{4}$. Since $\left|G / C_{G}(P)\right|$ is not divisible by $3, G / C_{G}(P)$ has a normal subgroup which is isomorphic to an extraspecial group of order 32. However, this group is not in the list of Theorem 3.17.

(3) For $p \in\{7,23\}$, if all cyclic subgroups of $P / Z(P)$ are conjugate, then $G / C_{G}(P)$ must contain an element of order 8 .

(4) Let $p=5$, so $G / C_{G}(P)$ has a normal subgroup $H \cong \mathrm{SL}(2,3)$. By our assumptions, $G / C_{G}(P)$ acts transitively on $Z(P)$. However, $\mathrm{SL}(2,3)$ can only act trivially on a cyclic group of order 5 since any element of order 4 of $\mathrm{SL}(2,3)$ is a product of two elements of order 3 . Thus, $\left|\left(G / C_{G}(P)\right) / H\right|$ must be even, whence there are more than three $\mathbb{Q}$-classes of 2-elements in $G$.

(5) For $p=11, G / C_{G}(P)$ contains a normal subgroup $H$ isomorphic to $\operatorname{SL}(2,3)$ or $\mathrm{SL}(2,5)$. As above, $G / C_{G}(P)$ must act transitively on $Z(P)$, but $\mathrm{SL}(2,3)$ and $\mathrm{SL}(2,5)$ can only act trivially on $C_{11}$. Thus, there are too many $\mathbb{Q}$ classes of 2-elements in $G$ if $G / C_{G}(P)$ acts transitively on $Z(P)$.

(6) If $p=19$ and all nontrivial elements of $Z(P)$ are conjugate in $G$, then $G / C_{G}(P)$ contains an element of order 9 . Moreover, $G / C_{G}(P)$ has a normal subgroup $H \cong \mathrm{SL}(2,5)$. Let $x \in G / C_{G}(P)$ be an element of order 9 . Suppose $x^{3} \in H$. Then, $x^{3}$ cannot centralize a Sylow 2-subgroup of $H$. Since the Sylow 2-subgroups of $H$ are isomorphic to $Q_{8}$, there is no Sylow 
2-subgroup of $H$ which is normalized by $x$. However, this is impossible since $H$ has exactly five Sylow 2-subgroups. Consequently, $x^{3} \notin H$, so $G / C_{G}(P)$ has at least two $\mathbb{Q}$-classes of elements of order 3 and at least one $\mathbb{Q}$-class of elements of order 9 . Hence, $G$ has at least four $\mathbb{Q}$-classes of 3-elements.

(7) For $p=29, G / C_{G}(P)$ contains a normal subgroup $H \cong \mathrm{SL}(2,5)$. Since $\mathrm{SL}(2,5)$ can only act trivially on $C_{29}, G$ contains at least four $\mathbb{Q}$-classes of 2-elements if all elements of $Z(P) \backslash\{1\}$ are conjugate in $G$.

(8) Finally, $G / C_{G}(P)$ has a normal subgroup $H \cong \mathrm{SL}(2,5)$ if $p=59$, so the same arguments as for $p=29$ apply.

We conclude that $G / C_{G}(P)$ cannot act on $P / Z(P)$ as one of the transitive linear groups. Thus, $R(G)$ cannot be representation-finite if $G$ has a nonabelian Sylow $p$-subgroup for some odd prime $p$.

From Theorems 3.2 and 3.20, it follows immediately that for an odd prime $p$, the Sylow $p$-subgroups of $G$ are cyclic or elementary-abelian if $R(G)$ is representationfinite.

\section{Structure of groups whose character rings are representation-finite}

In the previous section, we have focused on necessary conditions on $G$ for $R(G)$ having finite representation type. Now, we try to find sufficient conditions. For an odd prime $p$, we know that the Sylow $p$-subgroups of $G$ are cyclic of order $\leq p^{2}$ or elementary-abelian. We consider these two cases separately.

4.1. Cyclic Sylow subgroups. Let $p$ be a prime. For a cyclic group $C$ of order $p$ or $p^{2}$, the group ring of $C$ is representation-finite. Since the group ring and the character ring of a finite abelian group are isomorphic, $R(C)$ has finite representation type as well. This may suggest that $R(H)_{p}$ is representation-finite for a finite group $H$ with cyclic Sylow $p$-subgroups of order $p$ or $p^{2}$ respectively. We will show that this assertion holds if $H$ satisfies an additional condition.

We start with the possible number of $\mathbb{Q}$-classes in the rational $p^{\prime}$-sections of a finite group with cyclic Sylow $p$-subgroups.

Lemma 4.1. Let $P \in \operatorname{Syl}_{p}(G)$ be cyclic of order $p^{a}$ for some positive integer a. Suppose for any $x \in P$, two $p^{\prime}$-elements $y_{1}, y_{2} \in C_{G}(x)$ are $\mathbb{Q}$-conjugate in $G$ if and only if they are conjugate in $N_{G}(\langle x\rangle)$. Then, any rational $p^{\prime}$-section of $G$ has at most $a+1$ different $\mathbb{Q}$-classes.

Proof. Since $P$ is cyclic, any two $p$-elements of the same order are $\mathbb{Q}$-conjugate in $G$. Thus, it remains to show that $g, h \in G$ are $\mathbb{Q}$-conjugate if $g_{p}$ and $h_{p}$ as well as $g_{p^{\prime}}$ and $h_{p^{\prime}}$ are $\mathbb{Q}$-conjugate. 
Let $x \in P$. We consider the $\mathbb{Q}$-classes of a rational $p^{\prime}$-section in $N_{G}(\langle x\rangle)$ whose elements have $p$-parts which are $\mathbb{Q}$-conjugate to $x$, i.e. the $p$-parts have the same order as $x$. Let $x_{1} y_{1}$ and $x_{2} y_{2}$ be elements of such $\mathbb{Q}$-classes, that means $x_{1}, x_{2} \in$ $\langle x\rangle, y_{1}, y_{2} \in N_{G}(\langle x\rangle), p \nmid\left|\left\langle y_{1}\right\rangle\right|=\left|\left\langle y_{2}\right\rangle\right|, x_{i} y_{i}=y_{i} x_{i}$ for $i=1,2$, and $y_{1}$ is $\mathbb{Q}$-conjugate to $y_{2}$ in $N_{G}(\langle x\rangle)$. Then, there exist a $g \in N_{G}(\langle x\rangle)$ and some $\sigma \in$ $\operatorname{Gal}\left(\mathbb{Q}\left(\zeta_{\exp \left(N_{G}(\langle x\rangle)\right)}\right) / \mathbb{Q}\right)$ whose order is coprime to $p$ such that $\sigma\left(g y_{1} g^{-1}\right)=y_{2}$. Thus, we can find some $b \in \mathbb{Z}$ with $\operatorname{gcd}(b, p)=1$ such that $\sigma\left(g x_{1} y_{1} g^{-1}\right)=x^{b} y_{2}$ because any element of $N_{G}(\langle x\rangle)$ with order $|\langle x\rangle|$ already lies in $\langle x\rangle$. Moreover, since $\operatorname{gcd}\left(|\langle x\rangle|,\left|\left\langle y_{2}\right\rangle\right|\right)=1$, there is a $\tau \in \operatorname{Gal}\left(\mathbb{Q}\left(\zeta_{\exp \left(N_{G}(\langle x\rangle)\right)}\right) / \mathbb{Q}\right)$ with $\tau\left(x^{b} y_{2}\right)=x_{2} y_{2}$. This implies that $x_{1} y_{1}$ and $x_{2} y_{2}$ are contained in the same $\mathbb{Q}$-class of $N_{G}(\langle x\rangle)$. Hence, each rational $p^{\prime}$-section of $N_{G}(\langle x\rangle)$ has at most one $\mathbb{Q}$-class whose elements have $p$-parts with the same order as $x$.

Now, any element of $G$ which commutes with $x$ is already contained in $N_{G}(\langle x\rangle)$. Thus, any $\mathbb{Q}$-class of $G$ which contains an element with $p$-part $x$ also contains a $\mathbb{Q}$-class of $N_{G}(\langle x\rangle)$. On the other hand, any element of $G$ has a conjugate whose $p$-part lies in $P$. Since all elements of order $|\langle x\rangle|$ in $P$ are contained in $\langle x\rangle$, any $\mathbb{Q}$-class whose elements have $p$-parts with order $|\langle x\rangle|$ has a representative lying in $N_{G}(\langle x\rangle)$. But in $N_{G}(\langle x\rangle)$, any $p^{\prime}$-section has at most one $\mathbb{Q}$-class with elements whose $p$-parts have order $|\langle x\rangle|$ by the arguments above. Since two $p^{\prime}$-elements $y_{1}, y_{2} \in N_{G}(\langle x\rangle)$ are conjugate in $G$ if and only if they are already conjugate in $N_{G}(\langle x\rangle)$, we conclude that two elements lie in the same $\mathbb{Q}$-class of $G$ if their $p$-parts and $p^{\prime}$-parts are $\mathbb{Q}$-conjugate respectively.

If $G$ has cyclic Sylow subgroups and $\exp (G)$ is cube-free, then Lemma 4.1 yields that it only depends on the character values of $G$ whether $R(G)_{p}$ is representationfinite for some prime divisor $p$ of $|G|$.

Lemma 4.2. Let $P \in \operatorname{Syl}_{p}(G)$ be cyclic, and $g \in G$ be such that $\langle g\rangle \unlhd G$. If $\vartheta \in \operatorname{Irr}(\langle g\rangle)$ is invariant in $G$, then there is some $\chi \in \operatorname{Irr}(G)$ with $\chi_{\langle g\rangle}=e \vartheta$ such that $p \nmid e$.

Proof. We choose $Q \leq G$ such that $Q /\langle g\rangle \in \operatorname{Syl}_{p}(G /\langle g\rangle)$. Then, $Q /\langle g\rangle$ is cyclic, whence we can find some $\tilde{\vartheta} \in \operatorname{Irr}(Q)$ with $\tilde{\vartheta}_{\langle g\rangle}=\vartheta$ (see e.g. [29]). For the induced character $\tilde{\vartheta}^{G}$ of $\tilde{\vartheta}$ we obtain that $p$ does not divide $\tilde{\vartheta}^{G}(1)=|G: Q|$. Thus, $\tilde{\vartheta}^{G}$ has a constituent $\chi \in \operatorname{Irr}(G)$ whose degree is coprime to $p$. Frobenius reciprocity yields $\left\langle\chi_{Q}, \tilde{\vartheta}\right\rangle=\langle\chi, \tilde{\vartheta} G\rangle \neq 0$ so that $\left\langle\chi_{\langle g\rangle}, \vartheta\right\rangle \neq 0$. Now, $\vartheta$ is invariant in $G$ and we conclude $\chi_{\langle g\rangle}=e \vartheta$ with $e=\chi(1)$.

Lemma 4.3. Let $\mathcal{C}$ be a $\mathbb{Q}$-class of $G, g \in \mathcal{C}$, and $\chi_{1}, \ldots, \chi_{n}$ be the irreducible characters of $G$. Then, any element of $\mathbb{Z}_{(p)} \otimes \mathcal{O}_{\mathbb{Q}(\mathcal{C})}$ is already contained in $\mathbb{Z}_{(p)}\left[\chi_{1}(g), \ldots, \chi_{n}(g)\right]$. 
Proof. Let $\chi \in \operatorname{Irr}\left(N_{G}(\langle g\rangle)\right)$, and $\psi \in \operatorname{Irr}(\langle g\rangle)$ be a constituent of $\chi_{\langle g\rangle}$. By a theorem of Clifford, we get $\chi_{\langle g\rangle}=e_{\chi} \sum_{i=1}^{t} \psi_{i}$ where $\psi=\psi_{1}, \ldots, \psi_{t}$ are the conjugates of $\psi$ in $N_{G}(\langle g\rangle)$. We denote the inertia group of $\psi$ in $N_{G}(\langle g\rangle)$ by $I_{N_{G}(\langle g\rangle)}(\psi)$. From Lemma 4.2, we know that there exists some $\tilde{\psi} \in \operatorname{Irr}\left(I_{N_{G}(\langle g\rangle)}(\psi)\right)$ with $\tilde{\psi}_{\langle g\rangle}=e_{\psi} \psi$ and $p \nmid e_{\psi}$. By applying Clifford's theorem again, we obtain $\left(\tilde{\psi}^{N_{G}(\langle g\rangle)}\right)_{\langle g\rangle}=e_{\psi} \sum_{i=1}^{t} \psi_{i}$. Since $p \nmid e_{\psi}$, there is a function in $R\left(N_{G}(\langle g\rangle)\right)_{p}$ whose restriction to $\langle g\rangle$ coincides with $\sum_{i=1}^{t} \psi_{i}$.

We have shown the following: if $M_{1}, \ldots, M_{k}$ are the equivalence classes of irreducible characters of $\langle g\rangle$ with respect to $N_{G}(\langle g\rangle)$-conjugation and if $S_{j}:=\sum_{\varphi \in M_{j}} \varphi$ for $j=1, \ldots, k$, then the restriction of a character of $N_{G}(\langle g\rangle)$ to $\langle g\rangle$ is a $\mathbb{Z}$-linear combination of the $S_{j}$ and $S_{j} \in R\left(N_{G}(\langle g\rangle)\right)_{p}$ for $j=1, \ldots, k$. In particular, we obtain $\mathbb{Q}\left(\operatorname{cl}_{N_{G}(\langle g\rangle)}(g)\right)=\mathbb{Q}\left(S_{1}(g), \ldots, S_{k}(g)\right)$ and any $|\langle g\rangle|$ th root of unity is a summand of exactly one of the $S_{j}$ and appears only once there.

Let $j \in\{1, \ldots, k\}$. We have $S_{j}\left(n g n^{-1}\right)=S_{j}(g)$ for any $n \in N_{G}(\langle g\rangle)$ by construction. Moreover, $h g h^{-1}$ is certainly not contained in $N_{G}(\langle g\rangle)$ if $h \in G \backslash N_{G}(\langle g\rangle)$. Hence, for the induced function $S_{j}^{G}$, we get

$$
S_{j}^{G}(g)=\frac{1}{\left|N_{G}(\langle g\rangle)\right|} \sum_{h \in G} S_{j}\left(h g h^{-1}\right)^{\circ}=\frac{1}{\left|N_{G}(\langle g\rangle)\right|} \cdot\left|N_{G}(\langle g\rangle)\right| S_{j}(g)=S_{j}(g) .
$$

Since $S_{j}^{G} \in R(G)_{p}$, this yields $\mathbb{Z}_{(p)}\left[S_{1}(g), \ldots, S_{k}(g)\right] \subseteq \mathbb{Z}_{(p)}\left[\chi_{1}(g), \ldots, \chi_{n}(g)\right]$.

On the other hand, for any $i \in\{1, \ldots, n\}$, the restriction of $\chi_{i}$ to $N_{G}(\langle g\rangle)$ is a $\mathbb{Z}$-linear combination of irreducible characters of $N_{G}(\langle g\rangle)$. Therefore,

$$
\mathbb{Z}_{(p)}\left[\chi_{1}(g), \ldots, \chi_{n}(g)\right] \subseteq \mathbb{Z}_{(p)}\left[S_{1}(g), \ldots, S_{k}(g)\right]
$$

and we conclude

$$
\mathbb{Z}_{(p)}\left[S_{1}(g), \ldots, S_{k}(g)\right]=\mathbb{Z}_{(p)}\left[\chi_{1}(g), \ldots, \chi_{n}(g)\right] .
$$

This also shows $\mathbb{Z}_{(p)} \otimes \mathcal{O}_{\mathbb{Q}(\mathcal{C})}=\mathbb{Z}_{(p)} \otimes \mathcal{O}_{\mathbb{Q}\left(S_{1}(g), \ldots, S_{k}(g)\right)}$. Moreover, $\mathcal{O}_{\mathbb{Q}\left(S_{1}(g), \ldots, S_{k}(g)\right)}$ and $\mathbb{Z}\left[S_{1}(g), \ldots, S_{k}(g)\right]$ coincide because $\mathcal{O}_{\mathbb{Q}\left(\zeta_{|\langle g\rangle|}\right)}=\mathbb{Z}\left[\zeta_{|\langle g\rangle|}\right]$. This implies $\mathbb{Z}_{(p)} \otimes$ $\mathcal{O}_{\mathbb{Q}(\mathcal{C})}=\mathbb{Z}_{(p)}\left[S_{1}(g), \ldots, S_{k}(g)\right]$ and finally, we obtain

$$
\mathbb{Z}_{(p)} \otimes \mathcal{O}_{\mathbb{Q}(\mathcal{C})}=\mathbb{Z}_{(p)}\left[\chi_{1}(g), \ldots, \chi_{n}(g)\right]
$$

Theorem 4.4. Let $P \in \operatorname{Syl}_{p}(G)$ be cyclic of order $\leq p^{2}$. Suppose for any $x \in$ $P$, two $p^{\prime}$-elements $y_{1}, y_{2} \in C_{G}(x)$ are $\mathbb{Q}$-conjugate in $G$ if and only if they are conjugate in $N_{G}(\langle x\rangle)$. Then, $\mu_{0}$ and $\mu_{1}$ generate the $R(G)_{p}$-module $R(G)_{p}^{\prime} / R(G)_{p}$.

Proof. From Remark 2.8, we know that it suffices to show that for any rational $p^{\prime}$-section $\mathcal{S}$, the $R(G)_{p}$-module $\left(R(G)_{p}^{\prime} \cap \mathrm{Ch}_{\mathbb{Q}}(\mathcal{S})\right) /\left(R(G)_{p} \cap \mathrm{Ch}_{\mathbb{Q}}(\mathcal{S})\right)$ is generated by the restrictions of $\mu_{0}$ and $\mu_{1}$ on $\mathcal{S}$. 
Let $\mathcal{S}$ be an arbitrary rational $p^{\prime}$-section of $G$, and $y \in \mathcal{S}$ be a $p^{\prime}$-element. By Lemma 4.1, $\mathcal{S}$ contains at most three $\mathbb{Q}$-classes. From the proof of this lemma, we further obtain that any two elements of $\mathcal{S}$ of the same order are $\mathbb{Q}$-conjugate. Thus, there is a $p$-element $x \in G$ such that $y, x^{p} y$, and $x y$ are representatives of the $\mathbb{Q}$-classes in $\mathcal{S}$. We note that $x^{p} y=y$ or even $x y=y$ are possible. In such cases, $\mathcal{S}$ contains less than three $\mathbb{Q}$-classes.

We show that the $R(G)_{p}$-module $\left(R(G)_{p}^{\prime} \cap \mathrm{Ch}_{\mathbb{Q}}(\mathcal{S})\right) /\left(R(G)_{p} \cap \mathrm{Ch}_{\mathbb{Q}}(\mathcal{S})\right)$ is generated by $\mu_{0} \nu_{y}$ and $\mu_{1} \nu_{y}$. Since $\mu_{0}+\mu_{1}+\mu_{2}$ is the trivial character of $G$ and $\nu_{y} \in R(G)_{p}$ by Lemma 2.7, the function $\mu_{2} \nu_{y}=\nu_{y}-\mu_{0} \nu_{y}-\mu_{1} \nu_{y}$ lies in $\left\langle\mu_{0} \nu_{y}, \mu_{1} \nu_{y}\right\rangle$. Hence, for any $\mathbb{Q}$-class $\mathcal{C}$ contained in $\mathcal{S}$, the function $\varphi \in R(G)_{p}^{\prime} \cap \mathrm{Ch}_{\mathbb{Q}}(\mathcal{S})$ with $\varphi(g)=1$ for $g \in \mathcal{C}$ and $\varphi(g)=0$ for $g \in \mathcal{S} \backslash \mathcal{C}$ is also generated by $\mu_{0} \nu_{y}$ and $\mu_{1} \nu_{y}$. Thus, $\left\langle\mu_{0} \nu_{y}, \mu_{1} \nu_{y}\right\rangle$ contains the functions $\mu_{i} \nu_{y} \chi$ for $i=0,1,2$ and any $\chi \in \operatorname{Irr}(G)$.

That is why it suffices to show that for any $\mathbb{Q}$-class $\mathcal{C}$ of $\mathcal{S}$, the elements of $\mathbb{Z}_{(p)} \otimes$ $\mathcal{O}_{\mathbb{Q}(\mathcal{C})}$ are already contained in $\mathbb{Z}_{(p)}\left[\chi_{1}(g), \ldots, \chi_{n}(g)\right]$ where $g \in \mathcal{C}$ and $\chi_{1}, \ldots, \chi_{n}$ are the irreducible characters of $G$. But this is a direct consequence of Lemma 4.3 what completes the proof of the theorem.

After we have shown that there exists a generating system of size 2 for the $R(G)_{p^{-}}$ module $R(G)_{p}^{\prime} / R(G)_{p}$ where $G$ is a group satisfying the assumptions of the theorem above, we have to prove that the $R(G)_{p}$-module $\operatorname{rad}\left(R(G)_{p}^{\prime} / R(G)_{p}\right)$ is cyclic.

We consider the maximal order $R(G)_{p}^{\prime} \cong \bigoplus_{i=1}^{k} \mathbb{Z}_{(p)} \otimes \mathcal{O}_{\mathbb{Q}\left(\mathcal{C}_{i}\right)}$ where $\mathcal{C}_{1}, \ldots, \mathcal{C}_{k}$ are the $\mathbb{Q}$-classes of $G$. The maximal ideals of $R(G)_{p}^{\prime}$ are the ideals of the form $\left((1), \ldots, \mathfrak{p}_{i}, \ldots,(1)\right)$ with a maximal ideal $\mathfrak{p}_{i}$ of $\mathbb{Z}_{(p)} \otimes \mathcal{O}_{\mathbb{Q}\left(\mathcal{C}_{i}\right)}, i=1, \ldots, k$ (see e.g. [37]). Recall that any prime ideal of $\mathcal{O}_{\mathbb{Q}\left(\mathcal{C}_{i}\right)}$ contains exactly one prime $p \in \mathbb{Z}$ and that the maximal ideals of $\mathbb{Z}_{(p)} \otimes \mathcal{O}_{\mathbb{Q}\left(\mathcal{C}_{i}\right)}$ are in bijection to the prime ideals of $\mathcal{O}_{\mathbb{Q}\left(\mathcal{C}_{i}\right)}$ containing $p$. Since $\mathcal{O}_{\mathbb{Q}\left(\mathcal{C}_{i}\right)}$ has only finitely many prime ideals containing $p$, we conclude that $\mathbb{Z}_{(p)} \otimes \mathcal{O}_{\mathbb{Q}\left(\mathcal{C}_{i}\right)}$ is a principal ideal domain.

Now, we consider the cyclotomic fields $\mathbb{Q}\left(\zeta_{n}\right)$ with $n=p^{a} m, p^{a}>2$, and $p \nmid m$. Let $\mathfrak{P} \subseteq \mathcal{O}_{\mathbb{Q}\left(\zeta_{n}\right)}, \mathcal{P} \subseteq \mathcal{O}_{\mathbb{Q}\left(\zeta_{m}\right)}$, and $\mathfrak{p} \subseteq \mathcal{O}_{\mathbb{Q}\left(\zeta_{p^{a}}\right)}$ be prime ideals lying over $(p) \subseteq \mathbb{Z}$ such that $\mathfrak{P}$ lies over $\mathcal{P}$ as well as $\mathfrak{p}$. Since $p$ is unramified in $\mathcal{O}_{\mathbb{Q}\left(\zeta_{m}\right)}$, we obtain the following inequalities for the ramification indices:

$$
\begin{aligned}
\varphi\left(p^{a}\right) & =\left[\mathbb{Q}\left(\zeta_{n}\right): \mathbb{Q}\left(\zeta_{m}\right)\right] \geq e(\mathfrak{P} / \mathcal{P})=e(\mathfrak{P} / \mathcal{P}) e(\mathcal{P} /(p)) \\
& =e(\mathfrak{P} /(p))=e(\mathfrak{P} / \mathfrak{p}) e(\mathfrak{p} /(p))=e(\mathfrak{P} / \mathfrak{p}) \cdot \varphi\left(p^{a}\right) \geq \varphi\left(p^{a}\right) .
\end{aligned}
$$

Hence, $e(\mathfrak{P} /(p))=\varphi\left(p^{a}\right)$. Moreover, $\mathfrak{p}$ is fully ramified over $(p)$. Thus, $\mathfrak{p}$ is the only ideal of $\mathcal{O}_{\mathbb{Q}\left(\zeta_{p^{a}}\right)}$ which lies over $(p)$, so $\mathfrak{p}=\left(1-\zeta_{p^{a}}\right)$. Clearly, for $p^{a}=2$, we also obtain $\mathfrak{p}=(2)=\left(1-\zeta_{2}\right)$ if $\mathfrak{p}$ is the prime ideal in $\mathcal{O}_{\mathbb{Q}\left(\zeta_{2}\right)}=\mathbb{Z}$ lying over $(2)$.

The radical of $\mathbb{Z}_{(p)} \otimes \mathcal{O}_{\mathbb{Q}\left(\zeta_{n}\right)}$ is the intersection of all maximal ideals of $\mathbb{Z}_{(p)} \otimes$ $\mathcal{O}_{\mathbb{Q}\left(\zeta_{n}\right)}$. Since $\left(1-\zeta_{p^{a}}\right)$ is the only maximal ideal in $\mathbb{Z}_{(p)} \otimes \mathcal{O}_{\mathbb{Q}\left(\zeta_{p^{a}}\right)}$, the ideal $\left(1-\zeta_{p^{a}}\right)$ 
of $\mathbb{Z}_{(p)} \otimes \mathcal{O}_{\mathbb{Q}\left(\zeta_{n}\right)}$ is contained in this radical. Suppose, the radical is of the form $(\alpha)$ with $\left(1-\zeta_{p^{a}}\right) \subsetneq(\alpha)$. Then $(\alpha)$ would be the only maximal ideal in $\mathbb{Z}_{(p)} \otimes \mathcal{O}_{\mathbb{Q}(\alpha)}$. This would yield $e((\alpha) /(p))>e\left(\left(1-\zeta_{p^{a}}\right) /(p)\right)=\varphi\left(p^{a}\right)$ which is impossible.

Analogously, for a subfield $\mathbb{L}$ of $\mathbb{Q}\left(\zeta_{n}\right)$ and $\mathbb{K}=\mathbb{L} \cap \mathbb{Q}\left(\zeta_{p^{a}}\right)$, we obtain that a generator of the radical of $\mathbb{Z}_{(p)} \otimes \mathcal{O}_{\mathbb{K}}$ is also a generator of the radical of $\mathbb{Z}_{(p)} \otimes$ $\mathcal{O}_{\mathbb{L}}$. Let $g \in G$ be an element of order $n$, and $\lambda$ be a generator of $\operatorname{Irr}\left(\left\langle g^{n / p^{a}}\right\rangle\right)$. Then, $(\mathbb{1}-\lambda)(g)$ generates the radical of $\mathbb{Z}_{(p)} \otimes \mathcal{O}_{\mathbb{Q}\left(\mathrm{cl}_{\langle g\rangle}(g)\right)}$. Thus, the radical of $\mathbb{Z}_{(p)} \otimes \mathcal{O}_{\mathbb{Q}\left(\mathrm{cl}_{G}(g)\right)}$ is $\left(u((\mathbb{1}-\lambda)(g))^{b}\right)$ for a suitable unit $u \in \mathcal{O}_{\mathbb{Q}\left(\mathrm{cl}_{\langle g\rangle}(g)\right)}$ and a suitable positive integer $b$. These preparations will help us to prove the following theorem.

Theorem 4.5. Let $P \in \operatorname{Syl}_{p}(G)$ be cyclic of order $\leq p^{2}$. Suppose for any $x \in$ $P$, two $p^{\prime}$-elements $y_{1}, y_{2} \in C_{G}(x)$ are $\mathbb{Q}$-conjugate in $G$ if and only if they are conjugate in $N_{G}(\langle x\rangle)$. Then, the $R(G)_{p}$-module $\operatorname{rad}\left(R(G)_{p}^{\prime} / R(G)_{p}\right)$ is cyclic.

Proof. Let $g \in G$ be an element of $p^{\prime}$-order, and $\mathcal{S}$ be the rational $p^{\prime}$-section of $g$ in $G$. By Remark 2.8, it suffices to show that the $R(G)_{p}$-module

$$
\operatorname{rad}\left(\left(R(G)_{p}^{\prime} \cap \mathrm{Ch}_{\mathbb{Q}}(\mathcal{S})\right) /\left(R(G)_{p} \cap \mathrm{Ch}_{\mathbb{Q}}(\mathcal{S})\right)\right)
$$

is cyclic. Let $m:=|\langle g\rangle|$. We have the partition $\mathcal{S}=\mathcal{C}_{0} \cup \mathcal{C}_{1} \cup \mathcal{C}_{2}$ where $\mathcal{C}_{i}$ contains exactly the elements of order $p^{i} m$ of $\mathcal{S}, i=0,1,2$. Possibly, $\mathcal{C}_{2}=\emptyset$ or even $\mathcal{C}_{1}=\mathcal{C}_{2}=\emptyset$. The nonempty $\mathcal{C}_{i}$ are the $\mathbb{Q}$-classes of $G$ contained in $\mathcal{S}$ by Lemma 4.1 .

In the case $\mathcal{C}_{1}=\mathcal{C}_{2}=\emptyset$, Lemma 2.6 yields that there is nothing to show. Thus, we assume that $p$ divides $\left|C_{G}(g)\right|$, so $\mathcal{C}_{1} \neq \emptyset$.

By the assertions prior to this theorem, $(p)$ is the radical of $\mathbb{Z}_{(p)} \otimes \mathcal{O}_{\mathbb{Q}\left(\mathcal{C}_{0}\right)}$. Hence, $p \nu_{g} \mu_{0}$ generates $\operatorname{rad}\left(\left(R(G)_{p}^{\prime} \cap \mathrm{Ch}_{\mathbb{Q}}\left(\mathcal{C}_{0}\right)\right) /\left(R(G)_{p} \cap \mathrm{Ch}_{\mathbb{Q}}\left(\mathcal{C}_{0}\right)\right)\right)$. Certainly, the functions $p \nu_{g}, p \nu_{g} \mu_{1}$, and $p \nu_{g} \mu_{2}$ are also contained in $\operatorname{rad}\left(R(G)_{p}^{\prime} \cap \mathrm{Ch}_{\mathbb{Q}}(\mathcal{S})\right)$ (if $\mathcal{C}_{2}=\emptyset$, then $\left.\mu_{2}=0\right)$. Since $p \nu_{g} \mu_{0}=-p \nu_{g} \mu_{1}-p \nu_{g} \mu_{2}+p \nu_{g}$, we only have to find a generator of

$$
\operatorname{rad}\left(\left(R(G)_{p}^{\prime} \cap \mathrm{Ch}_{\mathbb{Q}}\left(\mathcal{C}_{1} \cup \mathcal{C}_{2}\right)\right) /\left(R(G)_{p} \cap \mathrm{Ch}_{\mathbb{Q}}\left(\mathcal{C}_{1} \cup \mathcal{C}_{2}\right)\right)\right) .
$$

At first, we suppose $\mathcal{C}_{2}=\emptyset$. Let $\chi_{1}, \ldots, \chi_{n}$ denote the irreducible characters of $G$, and $g_{1} \in \mathcal{C}_{1}$. By Lemma 4.3 , we obtain $\mathbb{Z}_{(p)} \otimes \mathcal{O}_{\mathbb{Q}\left(\mathcal{C}_{1}\right)}=\mathbb{Z}_{(p)}\left[\chi_{1}\left(g_{1}\right), \ldots, \chi_{n}\left(g_{1}\right)\right]$. Additionally, $\mathbb{Z}_{(p)} \otimes \mathcal{O}_{\mathbb{Q}\left(\mathcal{C}_{1}\right)}$ is a principal ideal domain, whence there exists an element $\pi$ which generates the radical of $\mathbb{Z}_{(p)} \otimes \mathcal{O}_{\mathbb{Q}\left(\mathcal{C}_{1}\right)}$. Therefore, the function $\eta \in \operatorname{rad}\left(R(G)_{p}^{\prime} \cap \mathrm{Ch}_{\mathbb{Q}}(\mathcal{S})\right)$ with $\eta\left(g_{1}\right)=\pi$ and $\eta(h)=0$ for $h \in G \backslash \mathcal{C}_{1}$ generates the $R(G)_{p}$-module

$$
\operatorname{rad}\left(\left(R(G)_{p}^{\prime} \cap \mathrm{Ch}_{\mathbb{Q}}(\mathcal{S})\right) /\left(R(G)_{p} \cap \mathrm{Ch}_{\mathbb{Q}}(\mathcal{S})\right)\right) .
$$

In particular, this module is cyclic.

Now, suppose $\mathcal{C}_{2} \neq \emptyset$. We choose $g_{1} \in \mathcal{C}_{1}$ and $g_{2} \in \mathcal{C}_{2}$ as well as generators $\pi_{1}, \pi_{2}$ of the radicals of $\mathbb{Z}_{(p)} \otimes \mathcal{O}_{\mathbb{Q}\left(\mathcal{C}_{1}\right)}$ and $\mathbb{Z}_{(p)} \otimes \mathcal{O}_{\mathbb{Q}\left(\mathcal{C}_{2}\right)}$ respectively. Again, there exists 
a class function $\eta_{1}$ with $\eta_{1}\left(g_{1}\right)=\pi_{1}$ and $\eta_{1}(h)=0$ for $h \in G \backslash \mathcal{C}_{1}$. It suffices to show that the class function $\eta_{2}$ with $\eta_{2}\left(g_{2}\right)=\pi_{2}$ and $\eta_{2}(h)=0$ for $h \in G \backslash \mathcal{C}_{2}$ is contained in the module generated by $\eta_{1}$.

To prove this, we show that the function $\varphi \in R(G)_{p}^{\prime}$ with $\varphi\left(g_{1}\right)=\pi_{1}, \varphi\left(g_{2}\right)=\pi_{2}$ and $\varphi(x)=0$ for $x \notin \mathcal{C}_{1} \cup \mathcal{C}_{2}$ (so $\varphi=\eta_{1}+\eta_{2}$ ) lies in $R(G)_{p}$. By a theorem of Brauer [4], this holds if and only if $\varphi_{E} \in R(E)_{p}$ for any elementary subgroup $E \leq G$. Since the Sylow $p$-subgroups of $G$ are cyclic and $\varphi(h)=0$ for $h \in G \backslash\left(\mathcal{C}_{1} \cup \mathcal{C}_{2}\right)$, we only have to consider the cyclic subgroups of $G$ which contain $g_{1}$. Moreover, we may assume that any of these cyclic subgroups whose order is divisible by $p^{2}$ contains $g_{2}$ as well.

Let $C<G$ be such a cyclic subgroup with $p^{2} \nmid|C|$. Then, $\left\langle g_{1}\right\rangle \in \operatorname{Syl}_{p}(C)$. Let $\lambda$ be a generator of $\operatorname{Irr}(C)$, so $\pi_{1}=u\left(\left(\mathbb{1}-\lambda^{|C| / p}\right)\left(g_{1}\right)\right)^{b}$ for suitable $u \in \mathcal{O}_{\mathbb{Q}\left(\zeta_{p}\right)}$ and $b \in \mathbb{N}$. Since $C$ is cyclic, $\left\{\chi\left(g_{1}\right): \chi \in \operatorname{Irr}(C)\right\}$ contains an integral basis of $\mathcal{O}_{\mathbb{Q}\left(\zeta_{p}\right)}$, whence there is a virtual character $\alpha$ of $C$ with $\alpha\left(g_{1}\right)=u$. Moreover, $\left(\mathbb{1}-\lambda^{|C| / p}\right)(h)=0$ for any $p$-regular element $h \in C$, and any $p$-singular element $h_{1} \in C$ which satisfies $\nu_{1_{C}}\left(h_{1}\right) \neq 0$ is a $\mathbb{Q}$-conjugate of $g_{1}$. This yields

$$
\varphi_{C}=\alpha \cdot \nu_{1_{C}} \cdot\left(\mathbb{1}-\lambda^{|C| / p}\right)^{b} \in R(C)_{p} .
$$

Finally, let $C \leq G$ with $g_{2} \in C$. We set $M:=\left\{i: 1 \leq i<p^{2}, g_{2}\right.$ is conjugate to $\left.g_{2}^{i}\right\}$. If $g_{2}$ and $g_{2}^{i}$ are conjugate in $G$, then $\chi_{C}\left(g_{2}\right)=\chi_{C}\left(g_{2}^{i}\right)$ holds for any character $\chi$ of $G$. Since the prime ideal of $\mathcal{O}_{\mathbb{Q}\left(\operatorname{cl}_{G}\left(g_{2}\right)\right)}$ containing $p$ is fully ramified, $\left(\left(1-\zeta_{p^{2}}\right)\left(1-\zeta_{p^{2}}^{i}\right)\right)$ divides $\left(\pi_{2}\right)$. Moreover, $\left(\pi_{2}\right)^{e}=(p)$ where $e$ coincides with the degree of the field extension $\mathbb{Q}\left(\operatorname{cl}_{G}\left(g_{2}\right)\right) / \mathbb{Q}$. By Remark 2.5, this degree equals the number of conjugacy classes of $G$ which are contained in the $\mathbb{Q}$-class of $g_{2}$. This implies $e=\left(p^{2}-p\right) /|M|$.

Thus, $\left(\pi_{2}\right)$ is a product of $|M|$ ideals of norm $p$ with respect to $\mathbb{Q}\left(\zeta_{p^{2}}\right) / \mathbb{Q}$, so $\left(\pi_{2}\right)=\left(\prod_{i \in M}\left(1-\zeta_{p^{2}}^{i}\right)\right)$. Analogously, $\left(\pi_{1}\right)=\left(\prod_{i \in M}\left(1-\zeta_{p}^{i}\right)\right)$. This product also runs over all $i \in M$ because $|M|$ divides $p-1$. Otherwise, $N_{G}\left(\left\langle g_{2}\right\rangle\right)$ would contain an element of order $p$ which does not lie in $\left\langle g_{2}\right\rangle$, so the Sylow $p$-subgroups of $G$ would not be cyclic.

Let $\lambda$ be a generator of $\operatorname{Irr}(C)$ such that $\lambda^{|C| / p^{2}}\left(g_{2}\right)=\zeta_{p^{2}}$. Then, $\mathbb{1}-\lambda^{j|C| / p^{2}} \in$ $R(C)$ for an arbitrary integer $j$ and we get

$$
\begin{aligned}
& \left(\mathbb{1}-\lambda^{j|C| / p^{2}}\right)\left(g_{1}\right)=1-\zeta_{p}^{j}, \quad\left(\mathbb{1}-\lambda^{j|C| / p^{2}}\right)\left(g_{2}\right)=1-\zeta_{p^{2}}^{j}, \quad \text { and } \\
& \left(\mathbb{1}-\lambda^{j|C| / p^{2}}\right)(x)=0 \quad \text { for any } p \text {-regular element } x \in C .
\end{aligned}
$$

Hence, $\nu_{1_{C}} \cdot \prod_{i \in M}\left(\mathbb{1}-\lambda^{i|C| / p^{2}}\right) \in R(C)_{p}$. That is why $\varphi_{C}$ is also an element of $R(C)_{p}$ and we conclude $\varphi \in R(G)_{p}$.

Theorems 4.4 and 4.5 yield the following result at once. 
Theorem 4.6. Let $G$ be a group with cyclic Sylow p-subgroups of order $\leq p^{2}$. Suppose for any p-element $x$, two $p^{\prime}$-elements $y_{1}, y_{2} \in C_{G}(x)$ are $\mathbb{Q}$-conjugate in $G$ if and only if they are conjugate in $N_{G}(\langle x\rangle)$. Then $R(G)_{p}$ is representation-finite.

In particular, the character ring of a group of cube-free order which has only cyclic Sylow subgroups has finite representation type.

4.2. Elementary-abelian Sylow subgroups. We have just seen that if $G$ has a cyclic Sylow $p$-subgroup $P$, then it mainly depends on the order of $P$ whether $R(G)_{p}$ is representation-finite. However, if $G$ has elementary-abelian Sylow $p$-subgroups, we need more information on the structure of $G$ to determine the representation type of $R(G)_{p}$. For instance, the character ring of $C_{2}^{2}$ has infinite representation type because $C_{2}^{2}$ has three $\mathbb{Q}$-classes of elements of order 2. Otherwise, $R\left(A_{4}\right)_{2}$ is representation-finite although the Sylow 2-subgroup of $A_{4}$ is elementary-abelian of rank 2 .

In the following, we try to find conditions on $G$ so that $R(G)$ has finite representation type. By Corollary $2.10, G$ has exactly one or exactly two $\mathbb{Q}$-classes of elements of order $p$. We consider both of the cases separately.

4.2.1. Groups with exactly one $\mathbb{Q}$-class of elements of order $p$. Let $p$ be a prime and $\mathfrak{p}$ be a maximal ideal in $\mathcal{O}_{\mathbb{Q}\left(\zeta_{|G|}\right)}$ containing $p$. It is well known that for any $\chi \in \operatorname{Irr}(G)$, we have $\chi(x) \equiv \chi(y)(\bmod \mathfrak{p})$ if $x, y \in G$ are elements with $x_{p^{\prime}}=y_{p^{\prime}}$ (see e.g. [29]). This result can be refined if $G$ has elementary-abelian Sylow $p$ subgroups and all cyclic subgroups of order $p$ of $G$ are conjugate. At first, we consider a special case where the refinement has been shown by K. Haberland. The general case will then follow easily.

For the proof of the refinement, we need a result due to Stickelberger which can be found in [5] or $[6,7]$ for instance.

Theorem 4.7 (Stickelberger). Let $q$ be a power of $p$, and $\ell$ be an integer with $0<\ell<q-1$, and $\ell=\sum_{j=0}^{n-1} \ell_{j} p^{j}, 0 \leq \ell_{j}<p$, be the $p$-adic representation of $\ell$. Moreover, let

$$
\Gamma_{\ell}:=\sum_{\alpha \in \mathbb{F}_{q}^{\times}} \alpha^{-\ell} \zeta^{\operatorname{Tr}(\alpha)}
$$

for some pth root of unity $\zeta$ with $\operatorname{Tr}=\operatorname{Tr}_{\mathbb{F}_{q} / \mathbb{F}_{p}}$. Then,

$$
\Gamma_{\ell} \equiv-\frac{(1-\zeta)^{\ell_{0}+\ldots+\ell_{n-1}}}{\ell_{0} ! \cdot \ldots \cdot \ell_{n-1} !} \quad\left(\bmod (1-\zeta)^{\ell_{0}+\ldots+\ell_{n-1}+1}\right)
$$

in $\mathbb{Z}_{(p)}[\zeta]$ 
Lemma 4.8 (Haberland). Let $p>2, k \geq 2, P$ be the additive group of $\mathbb{F}_{p^{k}}$, and $C$ be a subgroup of the multiplicative group of $\mathbb{F}_{p^{k}}$. Moreover, let

$$
G:=\left\{\left(\begin{array}{cc}
a & x \\
0 & 1
\end{array}\right): a \in C, x \in P\right\}
$$

$u \in G$ be an element of order $p$, and $\mathfrak{p}$ be the maximal ideal in $\mathcal{O}_{\mathbb{Q}\left(\operatorname{cl}_{G}(u)\right)}$ containing $p$. If $G$ has only one conjugacy class of subgroups of order $p$, then $\chi(1) \equiv \chi(u)$ $\left(\bmod \mathfrak{p}^{k}\right)$ for any $\chi \in \operatorname{Irr}(G)$.

Proof. We set

$$
V:=\left\{\left(\begin{array}{ll}
1 & x \\
0 & 1
\end{array}\right): x \in P\right\} \cong P \text { and } H:=\left\{\left(\begin{array}{ll}
a & 0 \\
0 & 1
\end{array}\right): a \in C\right\} \cong C .
$$

Obviously, $G$ is the semidirect product $V \rtimes H$ where $H$ acts transitively on the cyclic subgroups of $V$.

The number of cyclic subgroups of $V$ is $\left(p^{k}-1\right) /(p-1)$ since we can regard $P$ as an $\mathbb{F}_{p}$-vector space of dimension $k$. The stabilizer of $\mathbb{F}_{p}$ in the multiplicative group $\mathbb{F}_{p^{k}}^{\times}$is $C \cap \mathbb{F}_{p}^{\times}$. If $|C|=n$, then this stabilizer has $d:=\operatorname{gcd}(n, p-1)$ elements and the orbit of $\mathbb{F}_{p}$ has length $n / d$. Since $H$ acts transitively on the cyclic subgroups of $V$, the orbit length $n / d$ is a multiple of $\left(p^{k}-1\right) /(p-1)$, whence $n=\left(p^{k}-1\right) t /(p-1)$ with $d \mid t \leq p-1$.

It is easy to show that $G$ is a Frobenius group with kernel $V$ and complement $H$. Therefore, the conjugacy classes of $G$ have the following representatives:

(1) $1_{G}$,

(2) $P_{1}, \ldots, P_{(p-1) / t}$ where each $P_{i}$ is of the form $\left(\begin{array}{ll}1 & x \\ 0 & 1\end{array}\right)$ with $x \in \mathbb{F}_{p}$,

(3) $C_{1}, \ldots, C_{\left(p^{k}-1\right) t /(p-1)}$ where each $C_{j}$ is of the form $\left(\begin{array}{ll}a & 0 \\ 0 & 1\end{array}\right)$ with $a \in C$.

Since $G^{\prime}=V$, we get all linear characters of $G$ by extending the linear characters of $H$ : if $\lambda \in \operatorname{Irr}(C)$, we regard $\lambda$ as a linear character of $H$. Then, we get a linear character $\chi \in \operatorname{Irr}(G)$ by $\chi(g):=\lambda(h)$ where $g=v h$ with $v \in V$ and $h \in H$. Hence, for a linear character $\chi \in \operatorname{Irr}(G)$, we have already shown the assertion of the lemma.

Since $G$ is a Frobenius group with kernel $V$, we get the remaining $(p-1) / t$ irreducible characters of $G$ by inducing irreducible characters of $V$. At first, we consider the irreducible characters of $P$. Let $\mu_{p}$ denote the (complex) $p$ th roots of unity. The trace form

$$
P \times P \rightarrow \mathbb{F}_{p} \rightarrow \mu_{p}, \quad(x, y) \mapsto \operatorname{Tr}_{\mathbb{F}_{p^{k}} / \mathbb{F}_{p}}(x y) \mapsto \zeta_{p}^{\operatorname{Tr}(x y)}
$$


is nondegenerate, whence any irreducible character of $P$ can be expressed with the help of this trace form. More precisely, $\operatorname{Irr}(P)=\left\{\lambda_{x}: x \in P\right\}$ where $\lambda_{x}$ is given by

$$
\lambda_{x}: P \rightarrow \mu_{p}, \quad y \mapsto \zeta_{p}^{\operatorname{Tr}(x y)} .
$$

We write $\tilde{\lambda}_{x}$ for the respective character of $V$ which corresponds to $X:=\left(\begin{array}{ll}1 & x \\ 0 & 1\end{array}\right)$, i.e. $\tilde{\lambda}_{x}(Y):=\lambda_{x}(y)$ for $Y:=\left(\begin{array}{ll}1 & y \\ 0 & 1\end{array}\right)$. Since the conjugacy classes of elements of order $p$ in $G$ are represented by matrices whose upper right entry lies in $\mathbb{F}_{p}^{\times}$, we only have to consider the induced characters $\tilde{\lambda}_{x}^{G}$ with $X \in\left\{P_{1}, \ldots, P_{(p-1) / t}\right\}$, i.e. $x \in \mathbb{F}_{p}^{\times}$, and we only have to consider their values on the elements $Y:=\left(\begin{array}{ll}1 & y \\ 0 & 1\end{array}\right)$ with $Y \in\left\{P_{1}, \ldots, P_{(p-1) / t}\right\}$, i.e. $y \in \mathbb{F}_{p}^{\times}$. For the respective irreducible character $\chi_{x} \in \operatorname{Irr}(G)$, we obtain (besides $\chi_{x}(1)=\left(p^{k}-1\right) t /(p-1)$ and $\chi_{x}(g)=0, g \notin V$ )

$$
\begin{aligned}
\chi_{x}(Y) & =\tilde{\lambda}_{x}^{G}(Y)=\frac{1}{|V|} \sum_{g \in G} \tilde{\lambda}_{x}^{\circ}\left(g Y g^{-1}\right)=\sum_{g \in H} \tilde{\lambda}_{x}\left(g Y g^{-1}\right) \\
& =\sum_{a \in C} \lambda_{x}(a y)=\sum_{a \in C} \zeta_{p}^{\operatorname{Tr}(x a y)}=\sum_{a \in C} \zeta_{p}^{x y \operatorname{Tr}(a)}=\sum_{a \in C}\left(\zeta_{p}^{x y}\right)^{\operatorname{Tr}(a)} .
\end{aligned}
$$

Hence, we have to show $\chi_{x}\left(1_{G}\right) \equiv \chi_{x}(Y)\left(\bmod \mathfrak{p}^{k}\right)$. Since $\mathbb{Q}\left(\operatorname{cl}_{G}(Y)\right) / \mathbb{Q}$ is a field extension of degree $(p-1) / t$, we have $\mathfrak{p}=\left(1-\zeta_{p}\right)^{t} \cap \mathcal{O}_{\mathbb{Q}\left(\mathrm{cl}_{G}(Y)\right)}\left(\right.$ we regard $\left(1-\zeta_{p}\right)$ as an ideal in $\left.\mathcal{O}_{\mathbb{Q}\left(\zeta_{p}\right)}\right)$. Thus, we must show

$$
\frac{p^{k}-1}{p-1} \cdot t \equiv \sum_{a \in C}\left(\zeta_{p}^{x y}\right)^{\operatorname{Tr}(a)} \quad\left(\bmod \left(1-\zeta_{p}\right)^{t k}\right) .
$$

For $i=1, \ldots,(p-1) / t$, all $P_{i}$ lie in one cyclic group, so each of $\chi_{x}\left(P_{1}\right), \ldots$, $\chi_{x}\left(P_{(p-1) / t}\right)$ can be transferred by Galois automorphisms of $\mathbb{Q}\left(\operatorname{cl}_{G}(Y)\right) / \mathbb{Q}$ into each other. That is why it suffices to prove

$$
\frac{p^{k}-1}{p-1} \cdot t \equiv s_{1} \quad\left(\bmod \mathfrak{p}^{k}\right) \quad \text { for } s_{1}=\sum_{a \in C} \zeta_{p}^{\operatorname{Tr}(a)} .
$$

Let $M \leq \mathbb{F}_{p}^{\times}$be the subgroup which satisfies $\mathbb{F}_{p^{k}}^{\times}=\biguplus_{m \in M} m C$. For $m \in M$, we set

$$
s(m):=\sum_{a \in m^{-1} C} \zeta_{p}^{\operatorname{Tr}(a)}=\sum_{a \in C} \zeta_{p}^{m \operatorname{Tr}(a)} .
$$

Moreover, let $N \leq \operatorname{Irr}\left(\mathbb{F}_{p^{k}}^{\times}\right)$be the subgroup containing the irreducible characters of the multiplicative group of $\mathbb{F}_{p^{k}}$ which are trivial on $C$. Then, $|N|=(p-1) / t$. For $\psi \in N$, we have the Gaussian sum

$$
\Gamma(\psi):=\sum_{\alpha \in \mathbb{F}_{p^{x}}^{\times}} \psi(\alpha) \zeta_{p}^{\operatorname{Tr}(\alpha)}=\sum_{m \in M} \sum_{a \in C} \psi(m a) \zeta_{p}^{m \operatorname{Tr}(a)}=\sum_{m \in M} \psi(m) s(m) .
$$


That is why we can regard the function $\widetilde{\Gamma}$ with $\widetilde{\Gamma}(\psi):=\Gamma(\bar{\psi})$ as Fourier transform of $s$. This yields

$$
s_{1}=s\left(1_{G}\right)=\frac{1}{\frac{p-1}{t}} \sum_{\psi \in N} \overline{\psi\left(1_{G}\right)} \Gamma(\psi)=\frac{t}{p-1} \sum_{\psi \in N} \Gamma(\psi) .
$$

Since we only consider such characters $\psi$ which are trivial on $C$, we have $\psi(\alpha)=\alpha^{-\ell}$ for $\alpha \in \mathbb{F}_{p^{k}}^{\times}$and some

$$
\ell \in\left\{\frac{p^{k}-1}{p-1} \cdot t \cdot r: r \in\left\{0,1, \ldots, \frac{p-1}{t}-1\right\}\right\} .
$$

Since the trace is a surjective group homomorphism, we obtain

$$
\Gamma(\mathbb{1})=\sum_{a \in \mathbb{F}_{p^{k}}^{\times}} \zeta_{p}^{\operatorname{Tr}(a)}=-1+\sum_{a \in \mathbb{F}_{p^{k}}} \zeta_{p}^{\operatorname{Tr}(a)}=-1
$$

for the trivial character. Let $\psi \in N$ be a nontrivial character, so

$$
\Gamma(\psi)=\Gamma_{\ell}=\sum_{\alpha \in \mathbb{F}_{p^{k}}^{\times}} \alpha^{-\ell} \zeta_{p}^{\operatorname{Tr}(\alpha)}
$$

for some

$$
\ell \in\left\{\frac{p^{k}-1}{p-1} \cdot t \cdot r: r \in\left\{1,2, \ldots, \frac{p-1}{t}-1\right\}\right\} .
$$

Then, we can write

$$
\ell=\frac{p^{k}-1}{p-1} \cdot t \cdot r=\operatorname{tr}\left(1+\ldots+p^{k-1}\right)
$$

for an $r \in\{1,2, \ldots,(p-1) / t-1\}$. By Theorem 4.7, this implies

$$
\Gamma_{\ell} \equiv-\frac{\left(1-\zeta_{p}\right)^{k t r}}{((t r) !)^{k}} \quad\left(\bmod \left(1-\zeta_{p}\right)^{k t r+1}\right)
$$

where this is a congruence equation in $\mathbb{Z}_{(p)}\left[\zeta_{p}\right]$. Hence,

$\Gamma_{\ell} \equiv 0 \quad\left(\bmod \left(1-\zeta_{p}\right)^{k t+1}\right) \quad$ for $\quad \ell \in\left\{\frac{p^{k}-1}{p-1} \cdot t \cdot r: r \in\left\{2,3, \ldots, \frac{p-1}{t}-1\right\}\right\}$.

This gives

$$
\begin{aligned}
s_{1}-\frac{p^{k}-1}{p-1} \cdot t & =\frac{t}{p-1} \sum_{\psi \in N} \Gamma(\psi)-\frac{p^{k}-1}{p-1} \cdot t \equiv \frac{t}{p-1}\left(-1-\frac{\left(1-\zeta_{p}\right)^{k t}}{(t !)^{k}}-p^{k}+1\right) \\
& \equiv-\frac{t}{p-1} \cdot\left(\frac{\left(1-\zeta_{p}\right)^{t k}}{(t !)^{k}}+p^{k}\right) \quad\left(\bmod \left(1-\zeta_{p}\right)^{k t+1}\right) .
\end{aligned}
$$

Consequently, $\left(\left(1-\zeta_{p}\right)^{t k}\right)$ divides $\left(s_{1}-\left(p^{k}-1\right) t /(p-1)\right)$. Since $\mathfrak{p}=\left(\left(1-\zeta_{p}\right)^{t}\right) \cap$ $\mathcal{O}_{\mathbb{Q}\left(\operatorname{cl}_{G}(g)\right)}$, we obtain $\left(p^{k}-1\right) t /(p-1) \equiv s_{1}\left(\bmod \mathfrak{p}^{k}\right)$, that means $\chi_{x}\left(1_{G}\right) \equiv \chi_{x}(Y)$ $\left(\bmod \mathfrak{p}^{k}\right)$. 
Theorem 4.9. Let $P \in \operatorname{Syl}_{p}(G)$ be elementary-abelian of order $p^{k} \geq p^{3}$. If $G$ has only one $\mathbb{Q}$-class of elements of order $p$ and if the elements of order $p$ in $G$ are nonrational, then $R(G)$ has infinite representation type.

Proof. Clearly, the assumptions imply that $p$ is odd. Let $x$ be an element of order $p$. Due to the classification of transitive linear groups, $G$ contains a subgroup $H$ which is isomorphic to a subgroup of $\Gamma \mathrm{L}\left(p^{k}\right)$, contains $x$, and has exactly as many conjugacy classes of elements of order $p$ as $G$ has. Moreover, we assume that $H$ is a minimal subgroup of $G$ satisfying these properties.

At first, suppose $H$ is isomorphic to some group considered in Lemma 4.8. Then, for any irreducible character $\chi \in \operatorname{Irr}(H)$, we have $\chi(1) \equiv \chi(x)\left(\bmod \mathfrak{p}^{k}\right)$ where $\mathfrak{p}$ is the maximal ideal of $\mathcal{O}_{\mathbb{Q}\left(\mathrm{cl}_{H}(x)\right)}=\mathcal{O}_{\mathbb{Q}\left(\operatorname{cl}_{G}(x)\right)}$ containing $p$. Therefore, $\eta(1) \equiv \eta(x)$ $\left(\bmod \mathfrak{P}^{k}\right)$ for any $\eta \in R(H)_{p}$ where $\mathfrak{P}$ is the maximal ideal of $\mathbb{Z}_{(p)} \otimes \mathcal{O}_{\mathbb{Q}\left(\operatorname{cl}_{H}(x)\right)}$. The restriction of a class function of $G$ to $H$ is a class function of $H$, whence we even get $\eta(1) \equiv \eta(x)\left(\bmod \mathfrak{P}^{k}\right)$ for $\eta \in R(G)_{p}$.

Obviously, there are functions $\varphi_{1}, \varphi_{2} \in R(G)_{p}^{\prime}$ with $\varphi_{1}(x) \in \mathfrak{P} \backslash \mathfrak{P}^{2}, \varphi_{2}(x) \in$ $\mathfrak{P}^{2} \backslash \mathfrak{P}^{3}$, and $\varphi_{1}(g)=0=\varphi_{2}(g)$ for any $g \in G$ with $|\langle g\rangle| \neq p$. Suppose there exists an $\alpha \in \operatorname{rad}\left(R(G)_{p}^{\prime}\right)$ which generates the $R(G)_{p^{-}}$-module $\operatorname{rad}\left(R(G)_{p}^{\prime} / R(G)_{p}\right)$. Then, we can find $\eta_{1}, \eta_{2}, \xi_{1}, \xi_{2} \in R(G)_{p}$ such that $\eta_{1} \alpha+\xi_{1}=\varphi_{1}$ and $\eta_{2} \alpha+\xi_{2}=\varphi_{2}$. Since the values $\eta_{1}(1), \eta_{2}(1), \xi_{1}(1)$, and $\xi_{2}(1)$ are rational, each of them is either contained in $\mathfrak{P}^{k}$ or does not lie in $\mathfrak{P}$. Thus, each of $\eta_{1}(x), \eta_{2}(x), \xi_{1}(x)$, and $\xi_{2}(x)$ is also either contained in $\mathfrak{P}^{k}$ or does not lie in $\mathfrak{P}$ by Lemma 4.8 .

Now, $\eta_{1}(x) \alpha(x)+\xi_{1}(x)=\varphi_{1}(x) \in \mathfrak{P} \backslash \mathfrak{P}^{2}$. Since $\alpha(x) \in \mathfrak{P}$, we also get $\xi_{1}(x) \in \mathfrak{P}$, that means $\xi_{1}(x) \in \mathfrak{P}^{k}$. This yields $\alpha(x) \in \mathfrak{P} \backslash \mathfrak{P}^{2}$. Moreover, $\eta_{2}(x) \alpha(x)+\xi_{2}(x)=$ $\varphi_{2}(x) \in \mathfrak{P}^{2} \backslash \mathfrak{P}^{3}$. Since $\alpha(x) \in \mathfrak{P}$, we get $\xi_{2}(x) \in \mathfrak{P}$, so $\xi_{2}(x) \in \mathfrak{P}^{k}$. However, $\alpha$ does not lie in $\mathfrak{P}^{2}$, whence $\eta_{2}(x)$ is an element of $\mathfrak{P} \backslash \mathfrak{P}^{2}$. This is clearly impossible, that means the $R(G)_{p}$-module $\operatorname{rad}\left(R(G)_{p}^{\prime} / R(G)_{p}\right)$ is not cyclic and we conclude that $R(G)$ has infinite representation type.

We are left with the case that $H$ is not isomorphic to some group considered in Lemma 4.8. This means that $H$ is isomorphic to $K:=C_{p}^{k} \rtimes\left(C_{\left(p^{k}-1\right) t /((p-1) m)} \rtimes\right.$ $\left.C_{m}\right)$ for suitable positive integers $t$ and $k$ with $\operatorname{gcd}(k, p-1)|t| p-1$ and $m \mid k$. We show that $\chi(1) \equiv \chi(x)\left(\bmod \mathfrak{p}^{k}\right)$ also holds in this case for any $\chi \in \operatorname{Irr}(G)$.

Let $L$ be the subgroup of $\mathbb{F}_{p}^{k} \rtimes \Gamma \mathrm{L}\left(p^{k}\right)$ which is isomorphic to $C_{p}^{k} \rtimes C_{\left(p^{k}-1\right) t /(p-1)}$. The conjugacy classes of elements of order $p$ in $L$ coincide with the conjugacy classes of elements of order $p$ in

$$
M:=C_{p}^{k} \rtimes\left(C_{\frac{p^{k}-1}{p-1} t} \rtimes C_{m}\right) \leq \mathbb{F}_{p}^{k} \rtimes \Gamma \mathrm{L}\left(p^{k}\right)
$$


because the Galois automorphisms of $\mathbb{F}_{p^{k}}$ fix the subfield $\mathbb{F}_{p}$. Since $H$ has only one $\mathbb{Q}$-class of elements of order $p$, the conjugacy classes of elements of order $p$ in $M$ and $K$ coincide as well.

Let $\lambda \in \operatorname{Irr}\left(C_{p}^{k}\right)$. Then, $\lambda^{K}(y)=\lambda^{L}(y)$ because $|K|=|L|$ and the elements $y, z \in C_{p}^{k}$ are conjugate in $K$ if and only if they are conjugate in $L$. This implies $\lambda^{K}(1) \equiv \lambda^{K}(y)\left(\bmod \mathfrak{p}^{k}\right)$. Thus, for $\psi \in \operatorname{Irr}(H)$, we have $\psi(1) \equiv \psi(x)\left(\bmod \mathfrak{p}^{k}\right)$, whence $\chi(1) \equiv \chi(x)\left(\bmod \mathfrak{p}^{k}\right)$ for any $\chi \in \operatorname{Irr}(G)$. With the same arguments as in the case that $H$ is isomorphic to one of the groups considered in Lemma 4.8, we can show now that the $R(G)_{p}$-module $\operatorname{rad}\left(R(G)_{p}^{\prime} / R(G)_{p}\right)$ is not cyclic. This completes the proof of the theorem.

Corollary 4.10. Let $P \in \operatorname{Syl}_{p}(G)$ be elementary-abelian of order $p^{k} \geq p^{3}$. If $R(G)$ is representation-finite and $G$ has only one $\mathbb{Q}$-class of elements of order $p$, then $p^{k}-1$ is cube-free and all nontrivial elements of $P$ are conjugate in $G$.

Proof. Due to Theorem 4.9 and the classification of the finite transitive linear groups, one of the following applies.

(1) The action of $N_{G}(P) / C_{G}(P)$ on $P$ corresponds to the action of $\Gamma \mathrm{L}\left(p^{k}\right)$ on $\mathbb{F}_{p}^{k}$. Then, by Remark 3.13, the exponent of $G$ is divisible by $p^{k}-1$. Hence, $p^{k}-1$ must be cube-free if $R(G)$ is representation-finite.

(2) $P \cong C_{3}^{4}$ and $N_{G}(P) / C_{G}(P)$ acts as one of the sporadic transitive linear groups on $P$. Then, $G$ contains an extraspecial 2-group $H$ of order $2^{5}$. However, there are involutions in $H$ which do not commute, whence $H$ is no subgroup of a Suzuki 2-group. Therefore, the Sylow 2-subgroups of $G$ do not occur in the list of Theorem 3.17 , so $R(G)$ has infinite representation type.

On the other hand, for any elementary-abelian group $P$ of order $p^{k}>1$ with $p^{k}-1$ cube-free, there exists a group $G$ such that $P \in \operatorname{Syl}_{p}(G)$ and $R(G)$ is representationfinite: the cyclic group $C_{p^{k}-1}$ acts regularly on the nontrivial elements of $P$ if this action corresponds to the action of $\Gamma L_{0}\left(p^{k}\right)$ on $\mathbb{F}_{p}^{k}$. Moreover, $C_{p^{k}-1}$ is a cyclic Frobenius complement of $G:=P \rtimes C_{p^{k}-1}$, so $G$ satisfies the conditions above.

Since 8 divides $p^{2 k}-1$ for any positive integer $k$, Corollary 4.10 implies that the Sylow $p$-subgroups of $G$ cannot be elementary-abelian of even rank $>2$ if $G$ has only one $\mathbb{Q}$-class of elements of order $p$ and $R(G)$ is representation-finite. In the following, we will consider the case that the Sylow $p$-subgroups of $G$ are elementaryabelian of order $p^{2}$.

Proposition 4.11. Let $P \in \operatorname{Syl}_{p}(G)$ be elementary-abelian of order $p^{2}$, and $R(G)$ be representation-finite. If all subgroups of order $p$ are conjugate in $G$, then all elements of order $p$ are conjugate in $G$ and we have $p \in\{2,5,11,29,59\}$. 
Proof. If $p=2$, then the assertion is certainly satisfied. That is why we assume that $p$ is odd. Then, 8 divides $p^{2}-1$, so $G$ would possess an element of order 8 if $N_{G}(P) / C_{G}(P)$ would act on $P$ as $\Gamma \mathrm{L}\left(p^{2}\right)$ acts on $\mathbb{F}_{p}^{2}$. This would yield that $R(G)$ has infinite representation type.

Consequently, $G$ contains a subgroup which is isomorphic to a sporadic 2-transitive group and we obtain $p \in\{5,7,11,19,23,29,59\}$. For $p \in\{7,23\}$, by using GAP, we obtain immediately that $G$ contains an element of order 8 if all subgroups of order $p$ are conjugate in $G$.

Let $p=19$. The Sylow 3 -subgroups of $C_{19}^{2} \rtimes\left(C_{9} \times \mathrm{SL}(2,5)\right)$ are isomorphic to $C_{9} \times C_{3}$. Thus, $R(G)$ has infinite representation type if $G$ has such a subgroup.

Both of the groups $H_{1}:=C_{19}^{2} \rtimes\left(C_{3} \times \mathrm{SL}(2,5)\right)$ and $H_{2}:=C_{19}^{2} \rtimes \mathrm{SL}(2,5)$ contain three conjugacy classes of elements of order 19 respectively. Let $\mathcal{C}$ be one of these classes, $x \in \mathcal{C}$, and $\mathfrak{p}$ be the maximal ideal in $\mathcal{O}_{\mathbb{Q}(\mathcal{C})}$ containing 19. With the help of GAP, we can verify that $\chi(1) \equiv \chi(x)\left(\bmod \mathfrak{p}^{2}\right)$ holds for any $\chi \in \operatorname{Irr}\left(H_{1}\right)$ or $\chi \in$ $\operatorname{Irr}\left(H_{2}\right)$ respectively. Additionally, there exist irreducible characters $\psi_{1} \in \operatorname{Irr}\left(H_{1}\right)$ and $\psi_{2} \in \operatorname{Irr}\left(H_{2}\right)$ and some $\sigma \in \operatorname{Gal}(\mathbb{Q}(\mathcal{C}) / \mathbb{Q})$ such that any irreducible character of $H_{i}$ is rational on $x$ or is one of $\psi_{i}(x), \sigma\left(\psi_{i}(x)\right), \sigma^{2}\left(\psi_{i}(x)\right), i=1,2$.

Now, we are able to show that the $R\left(H_{i}\right)_{19}$-module $\operatorname{rad}\left(R\left(H_{i}\right)_{19}^{\prime} / R\left(H_{i}\right)_{19}\right), i \in$ $\{1,2\}$, is not cyclic. Suppose $\alpha$ is a generator of this module. Then, $\alpha(x) \in \mathfrak{P} \backslash \mathfrak{P}^{2}$ where $\mathfrak{P}$ is the maximal ideal of $\mathbb{Z}_{(19)} \otimes \mathcal{O}_{\mathbb{Q}(\mathcal{C})}$. However, for any $\eta \in\langle\alpha\rangle$, we have $\eta(x)=r \cdot \alpha(x)+s$ with suitable $r, s \in \mathbb{Z}_{(19)}$. Thus, there is no such $\eta$ with $\eta(x)=\sigma(\alpha(x))$. Otherwise, the class function $\varphi$ with $\varphi(x)=\sigma(\alpha(x))$ and $\varphi(h)=0$ for $h \in H_{i}$ of order $\neq 19$ lies in $\operatorname{rad}\left(R\left(H_{i}\right)_{19}^{\prime}\right) \backslash R\left(H_{i}\right)_{19}$. In particular, the $R(G)_{19}$-module $\operatorname{rad}\left(R(G)_{19}^{\prime} / R(G)_{19}\right)$ is not cyclic so that $R(G)$ would have infinite representation type.

Each of the proper subgroups $\neq H_{1}, H_{2}$ of $C_{19}^{2} \rtimes\left(C_{9} \times \mathrm{SL}(2,5)\right)$ whose order is divisible by $19^{2}$ has more than one $\mathbb{Q}$-class of elements of order 19 . Hence, the Sylow 19-subgroups of $G$ cannot be elementary-abelian of rank 2 .

Next, let $p=5$. Since there is no proper subgroup of $C_{5}^{2} \rtimes \mathrm{SL}(2,3)$ containing the normal subgroup of order 25 in which all cyclic subgroups of order 5 are conjugate, there is nothing to show in this case.

Suppose $p=11$. The group $C_{11}^{2} \rtimes \mathrm{SL}(2,5)$ does not have a proper subgroup with only one $\mathbb{Q}$-class of elements of order 11 whose order is divisible by $11^{2}$, so there is again nothing to show. However, $C_{11}^{2} \rtimes\left(C_{5} \times \mathrm{SL}(2,3)\right)$ contains exactly one proper subgroup $H$ with such properties: the subgroup isomorphic to $C_{11}^{2} \rtimes \mathrm{SL}(2,3)$. Let $\mathfrak{p}$ denote the maximal ideal in $\mathcal{O}_{\mathbb{Q}\left(\operatorname{cl}_{H}(x)\right)}$ containing 11. Then, for $x \in H$ of order 11 and $\chi \in \operatorname{Irr}(H)$, we get $\chi(1) \equiv \chi(x)\left(\bmod \mathfrak{p}^{3}\right)$. Similar to the proof of Theorem 4.9, we conclude that the $R(H)_{11}$-module $\operatorname{rad}\left(R(H)_{11}^{\prime} / R(H)_{11}\right)$ is not cyclic. Thus, the 
$R(G)_{11}$-module $\operatorname{rad}\left(R(G)_{11}^{\prime} / R(G)_{11}\right)$ can only be cyclic if $G$ contains a subgroup isomorphic to $C_{11}^{2} \rtimes\left(C_{5} \times \mathrm{SL}(2,3)\right)$ besides $H$.

The cases $p=29$ and $p=59$ are analogous. The only proper subgroup $H$ of $C_{29}^{2} \rtimes\left(C_{7} \times \mathrm{SL}(2,5)\right)$ with a single $\mathbb{Q}$-class of elements of order 29 whose order is divisible by $29^{2}$ is isomorphic to $C_{29}^{2} \rtimes \mathrm{SL}(2,5)$. If $x \in H$ is of order $29, \chi \in \operatorname{Irr}(H)$, and $\mathfrak{p}$ denotes the maximal ideal in $\mathcal{O}_{\mathbb{Q}\left(\operatorname{cl}_{H}(x)\right)}$ containing 29 , then we even get $\chi(1) \equiv \chi(x)\left(\bmod \mathfrak{p}^{6}\right)$.

Finally, there is only one proper subgroup $H$ in $C_{59}^{2} \rtimes\left(C_{29} \times \mathrm{SL}(2,5)\right)$ whose order is divisible by $59^{2}$ and whose cyclic subgroups of order 59 are all conjugate. This subgroup is isomorphic to $C_{59}^{2} \rtimes \mathrm{SL}(2,5)$. For $x \in H$ of order $59, \chi \in \operatorname{Irr}(H)$, and the maximal ideal $\mathfrak{p}$ in $\mathcal{O}_{\mathbb{Q}\left(\operatorname{cl}_{H}(x)\right)}$ containing 59 , we obtain $\chi(1) \equiv \chi(x)\left(\bmod \mathfrak{p}^{6}\right)$.

For any elementary-abelian $p$-group $P$ of order $p^{2}$ with $p \in\{2,5,11,29,59\}$, there is in fact a group $G$ such that $R(G)$ is representation-finite and $P \in \operatorname{Syl}_{p}(G)$. Certainly, $R\left(A_{4}\right)$ is representation-finite. Moreover, it is easy to verify that the character rings of the sporadic 2-transitive groups

$$
\begin{aligned}
& C_{5}^{2} \rtimes \mathrm{SL}(2,3), \quad C_{11}^{2} \rtimes\left(C_{5} \times \mathrm{SL}(2,3)\right), \quad C_{11}^{2} \rtimes \mathrm{SL}(2,5), \\
& C_{29}^{2} \rtimes\left(C_{7} \times \mathrm{SL}(2,5)\right) \quad \text { and } \quad C_{59}^{2} \rtimes\left(C_{29} \times \mathrm{SL}(2,5)\right)
\end{aligned}
$$

have finite representation type respectively because all nontrivial elements of the respective elementary-abelian normal subgroup are conjugate.

Furthermore, the arguments in the proof of Proposition 4.11 yield that $G$ must

contain one of these 2-transitive groups if $P \in \operatorname{Syl}_{p}(G)$ has order $p^{2}$, all subgroups of order $p$ are conjugate in $G$, and $R(G)$ is representation-finite.

Up to now, we have only considered conditions on the elements of order $p$ of a group $G$ with elementary-abelian Sylow $p$-subgroup if $R(G)_{p}$ is representationfinite. In general, these conditions do not suffice to determine whether $R(G)_{p}$ has finite representation type. This can be illustrated by the following examples.

\section{Example 4.12.}

(1) Let $G=\left(C_{5}^{2} \times C_{11}^{2}\right) \rtimes C_{12}$ where $C_{12}$ acts on $C_{5}^{2}$ as the subgroup of index 2 in $\Gamma \mathrm{L}_{0}\left(5^{2}\right)$ on $\mathbb{F}_{5}^{2}$, and $C_{12}$ acts on $C_{5}^{2}$ as the subgroup of index 10 in $\Gamma L_{0}\left(11^{2}\right)$ on $\mathbb{F}_{11}^{2}$. Then, for any prime divisor $p$ of $|G|$, there are at most three $\mathbb{Q}$-classes of p-elements in $G$. However, $G$ contains 240 conjugacy classes of elements of order 55 . Hence, for $x \in G$ of order 5 , there are at least three $\mathbb{Q}$-classes of elements of order 55 in the rational $11^{\prime}$-section of $x$. Consequently, any generating system of the $R(G)_{11}$-module $R(G)_{11}^{\prime} / R(G)_{11}$ possesses at least three elements. This cannot be shown if one considers only the elements of order 11. 
(2) Let $G=\left(C_{3}^{3} \times C_{5}\right) \rtimes C_{52}$ where the cyclic subgroup of order 52 acts transitively on the nontrivial elements of $C_{3}^{3}$ as well as the nontrivial elements of $C_{5}$. Then, $G$ has only one conjugacy class of elements of order 3 and elements of order 5 respectively. However, there are two conjugacy classes of elements of order 15 in $G$. The only nonreal values of irreducible characters of $G$ on these two classes are $(1 \pm 3 \sqrt{-15}) / 2$. That is why the $R(G)_{3}$-module $\operatorname{rad}\left(R(G)_{3}^{\prime} / R(G)_{3}\right)$ is not cyclic, whence $R(G)$ has infinite representation type.

The second example shows that $R(G)$ can have infinite representation type even if $G$ has a normal subgroup $N$ with $\operatorname{gcd}(|N|,|G: N|)=1$ such that $R(N)$ as well as $R(G / N)$ have finite representation type (choose $N=C_{5}$ in the example).

On the other hand, it is clear that for a normal subgroup $N \unlhd G, R(N)$ can have infinite representation type if $R(G)$ is representation-finite (choose $G=A_{4}, N=C_{2}^{2}$ for instance). Hence, there will not be any reduction theorems which connect the representation type of $R(G)$ and the representation type of the character ring of some normal subgroup of $G$.

4.2.2. Groups with exactly two $\mathbb{Q}$-classes of elements of order $p$. The case that $G$ has exactly two $\mathbb{Q}$-classes of elements of order $p$ is more complicated than the previous case. The reason for this is that only if we assume $G$ to be solvable we have an analogous classification to Theorem 3.11 which applies in our case. Nonetheless, we can prove some general results. Since a finite group with an elementary-abelian Sylow 2-subgroup cannot contain exactly two conjugacy classes of involutions, we assume that $p$ is an odd prime in this section.

Lemma 4.13. Let $P \in \operatorname{Syl}_{p}(G)$ be elementary-abelian of order $p^{k} \geq p^{3}$. If $G$ has two rational conjugacy classes of elements of order $p$, then $R(G)$ is not representation-finite.

Proof. It is clear that $R(G)$ has infinite representation type if $G$ has more than three $\mathbb{Q}$-classes of $p$-elements. That is why we suppose that $\mathcal{C}_{1}$ and $\mathcal{C}_{2}$ are the $\mathbb{Q}$ classes of $G$ containing elements of order $p$. Moreover, the elements of $\mathcal{C}_{1}$ and $\mathcal{C}_{2}$ are rational.

We are going to show that the $R(G)_{p}$-module $\operatorname{rad}\left(R(G)_{p}^{\prime} / R(G)_{p}\right)$ is not cyclic. Certainly, the function $\varphi_{1}$ with $\varphi_{1}(1)=p$ and $\varphi_{1}(g)=0$ for $g \in G \backslash\{1\}$ lies in $R(G)_{p}^{\prime} \backslash R(G)_{p}$. If $\varrho$ is the regular character of $G$, then $\varphi_{1}=p /|G| \cdot \varrho$. Therefore, $p^{k-2} \varphi_{1} \in R(G)_{p}^{\prime} \backslash R(G)_{p}$ and we have $\left\langle\varphi_{1}, \mathbb{1}\right\rangle=p /|G|$. 
Let $x \in P \cap \mathcal{C}_{1}$. By Lemma 2.13, there exists a character $\psi$ of $G$ satisfying

$$
\begin{aligned}
& \psi(g)= \begin{cases}\frac{\left|C_{G}(g)\right|}{|\langle x\rangle|} \cdot \sum_{\sigma \in \operatorname{Gal}\left(\mathbb{Q}(\zeta|\langle g\rangle|) / \mathbb{Q}\left(\operatorname{cl}_{G}(g)\right)\right)}^{\sigma(1)} & , g \sim_{G} x^{k} \text { for some } k \in \mathbb{Z} \\
0, & \text { else }\end{cases} \\
& = \begin{cases}\frac{\left|C_{G}(g)\right|}{|\langle x\rangle|} \cdot \frac{\left|N_{G}(\langle g\rangle)\right|}{\left|C_{G}(g)\right|}, & g \sim_{G} x^{k} \text { for some } k \in \mathbb{Z} \\
0, & \text { else }\end{cases} \\
& =\left\{\begin{array}{ll}
\frac{\left|N_{G}(\langle g\rangle)\right|}{|\langle x\rangle|}, & g \sim_{G} x^{k} \text { for some } k \in \mathbb{Z} \\
0, & \text { else }
\end{array} .\right.
\end{aligned}
$$

Lemma 2.13 further yields $\langle\psi, \mathbb{1}\rangle=1$. Thus, the function

$$
\varphi_{2}:=\frac{p^{2}}{\left|N_{G}(\langle x\rangle)\right|}\left(\psi-\frac{|G|}{p^{2}} \varphi_{1}\right)
$$

i.e. $\varphi_{2}(g)=p$ for $g \sim_{G} x$ and $\varphi_{2}(g)=0$ for $g \nsim_{G} x$, is also contained in $R(G)_{p}^{\prime} \backslash$ $R(G)_{p}$ and we obtain

$$
\left\langle\varphi_{2}, \mathbb{1}\right\rangle=\frac{p^{2}}{\left|N_{G}(\langle x\rangle)\right|}-\frac{|G|}{\left|N_{G}(\langle x\rangle)\right|} \cdot \frac{p}{|G|}=\frac{p^{2}}{\left|N_{G}(\langle x\rangle)\right|}-\frac{p}{\left|N_{G}(\langle x\rangle)\right|}=\frac{p}{\left|C_{G}(x)\right|} .
$$

Similarly, for $y \in P \cap \mathcal{C}_{2}$, the function $\varphi_{3}$ with $\varphi_{3}(g)=p$ for $g \sim_{G} y$ and $\varphi_{3}(g)=0$ for $g \nsim_{G} y$ lies in $R(G)_{p}^{\prime} \backslash R(G)_{p}$.

Let $\chi \in \operatorname{Irr}(G)$. Then, $p$ divides $\chi(x)-\chi(1)$ because $\operatorname{cl}_{G}(x)$ is rational. Suppose

$$
\chi(x)-\chi(1) \equiv r p \quad\left(\bmod p^{k}\right)
$$

for some $r \in \mathbb{Z}$ with $\operatorname{gcd}(r, p)=1$. Then, we find $\varphi \in R(G)_{p}$ such that $0 \leq$ $(\chi-\varphi)(1)<p^{k},(\chi-\varphi)(x)-(\chi-\varphi)(1) \equiv \operatorname{rp}\left(\bmod p^{k}\right)$, and $(\chi-\varphi)(y)=0$ since $\mathbb{1}, p^{k-1} \varphi_{1}$, and $p^{k-1} \varphi_{2}$ lie in $R(G)$. For the function $\eta:=\nu_{1}(\chi-\varphi) \in R(G)_{p}$, this gives

$$
\eta(g)= \begin{cases}a p, & g=1 \\ b p, & g \sim_{G} x \\ 0, & \text { else }\end{cases}
$$

where $a, b \in\left\{0,1, \ldots, p^{k-1}-1\right\}$ are integers such that $b p-a p \equiv r p\left(\bmod p^{k}\right)$. Hence, we can write $\eta=a \varphi_{1}+b \varphi_{2}$, so

$$
\langle\eta, \mathbb{1}\rangle=a\left\langle\varphi_{1}, \mathbb{1}\right\rangle+b\left\langle\varphi_{2}, \mathbb{1}\right\rangle=\frac{a p}{|G|}+\frac{b p}{\left|C_{G}(x)\right|}=\frac{p\left(a+b \cdot\left|\mathrm{cl}_{G}(x)\right|\right)}{|G|} .
$$

Since $\eta \in R(G)_{p}$, the numerator $p\left(a+b \cdot\left|\operatorname{cl}_{G}(x)\right|\right)$ is divisible by $p^{k}$, whence

$$
a+b \cdot\left|\mathrm{cl}_{G}(x)\right| \equiv 0 \quad\left(\bmod p^{k-1}\right) .
$$


Additionally,

$$
\langle\eta, \chi\rangle=\frac{1}{|G|} \cdot\left(\eta(1) \chi(1)+\left|\mathrm{cl}_{G}(x)\right| \eta(x) \chi(x)\right)=\frac{a p \chi(1)+\left|\mathrm{cl}_{G}(x)\right| \cdot b p \chi(x)}{|G|} .
$$

Here, the numerator is also divisible by $p^{k}$, so

$$
a \chi(1)+\left|\mathrm{cl}_{G}(x)\right| b \chi(x) \equiv 0 \quad\left(\bmod p^{k-1}\right) .
$$

According to the equations (1) and (2), we replace $\chi(1)$ by $\chi(x)-r p$ and $a$ by $-b\left|\mathrm{cl}_{G}(x)\right|$. This yields

$$
0 \equiv-b\left|\mathrm{cl}_{G}(x)\right| \cdot(\chi(x)-r p)+b\left|\mathrm{cl}_{G}(x)\right| \chi(x) \equiv p b r\left|\mathrm{cl}_{G}(x)\right| \quad\left(\bmod p^{k-1}\right) .
$$

Now, $\left|\mathrm{cl}_{G}(x)\right|=\left|G: C_{G}(x)\right|$ is coprime to $p$ because $P$ is abelian. Since $r$ is also coprime to $p$ by assumption, we obtain $b \equiv 0\left(\bmod p^{k-2}\right)$. By applying equation (2) again, we also get $a \equiv 0\left(\bmod p^{k-2}\right)$. However, this is a contradiction to $b p-a p \equiv$ $r p\left(\bmod p^{k}\right)$.

Hence, for every $\chi \in \operatorname{Irr}(G)$, we have $\chi(1) \equiv \chi(x)\left(\bmod p^{2}\right)$. Analogously, we obtain $\chi(1) \equiv \chi(y)\left(\bmod p^{2}\right)$ for $\chi \in \operatorname{Irr}(G)$. This implies $\eta(1) \equiv \eta(x) \equiv \eta(y)$ $\left(\bmod p^{2}\right)$ since $\eta \in R(G)_{p}$.

Suppose the $R(G)_{p}$-module $\operatorname{rad}\left(R(G)_{p}^{\prime} / R(G)_{p}\right)$ is generated by $\alpha \in \operatorname{rad}\left(R(G)_{p}^{\prime}\right) \backslash$ $R(G)_{p}$. Then, we can find $\eta_{1}, \eta_{2}, \eta_{3}, \xi_{1}, \xi_{2}, \xi_{3} \in R(G)_{p}$ with $\eta_{1} \alpha+\xi_{1}=\varphi_{1}, \eta_{2} \alpha+\xi_{2}=$ $\varphi_{2}$, and $\eta_{3} \alpha+\xi_{3}=\varphi_{3}$. We consider the ring homomorphism

$$
F: R(G)_{p}^{\prime} \rightarrow\left(\mathbb{Z} / p^{2} \mathbb{Z}\right)^{3}, \quad \vartheta \mapsto\left(\vartheta(1)+p^{2} \mathbb{Z}, \vartheta(x)+p^{2} \mathbb{Z}, \vartheta(y)+p^{2} \mathbb{Z}\right) .
$$

Since $\eta_{i}$ and $\xi_{i}$ lie in $R(G)_{p}$, there exist $a_{1}, a_{2}, a_{3}, b_{1}, b_{2}, b_{3} \in \mathbb{Z}$ such that $F\left(\eta_{i}\right)=$ $\left(a_{i}+p^{2} \mathbb{Z}, a_{i}+p^{2} \mathbb{Z}, a_{i}+p^{2} \mathbb{Z}\right)$ and $F\left(\xi_{i}\right)=\left(b_{i}+p^{2} \mathbb{Z}, b_{i}+p^{2} \mathbb{Z}, b_{i}+p^{2} \mathbb{Z}\right)$ for $i=1,2,3$. Let $F(\alpha)=\left(x+p^{2} \mathbb{Z}, y+p^{2} \mathbb{Z}, z+p^{2} \mathbb{Z}\right)$ with $x, y, z \in \mathbb{Z}$. Then, we have the equations

$$
\begin{array}{ll}
\left(a_{1} x+b_{1}, a_{1} y+b_{1}, a_{1} z+b_{1}\right) \equiv(p, 0,0) & \left(\bmod p^{2}\right), \\
\left(a_{2} x+b_{2}, a_{2} y+b_{2}, a_{2} z+b_{2}\right) \equiv(0, p, 0) & \left(\bmod p^{2}\right), \\
\left(a_{3} x+b_{3}, a_{3} y+b_{3}, a_{3} z+b_{3}\right) \equiv(0,0, p) & \left(\bmod p^{2}\right) .
\end{array}
$$

From equation (3), we obtain $a_{1} y+b_{1} \equiv a_{1} z+b_{1}$, i.e. $a_{1}(y-z) \equiv 0\left(\bmod p^{2}\right)$. Now, $y-z$ is not divisible by $p^{2}$ since $a_{2}(y-z) \equiv p\left(\bmod p^{2}\right)$ by equation $(4)$. This gives $a_{1}=p \tilde{a}_{1}$ and $b_{1}=p \tilde{b}_{1}$ with $\tilde{a}_{1}, \tilde{b}_{1} \in \mathbb{Z}$.

A similar analysis of the equations (4) and (5) yields $a_{2}=p \tilde{a}_{2}, b_{2}=p \tilde{b}_{2}, a_{3}=$ $p \tilde{a}_{3}$, and $b_{3}=p \tilde{b}_{3}$ with $\tilde{a}_{2}, \tilde{b}_{2}, \tilde{a}_{3}, \tilde{b}_{3} \in \mathbb{Z}$. But then, the equations

$$
\begin{array}{ll}
\left(\tilde{a}_{1} x+\tilde{b}_{1}, \tilde{a}_{1} y+\tilde{b}_{1}, \tilde{a}_{1} z+\tilde{b}_{1}\right) \equiv(1,0,0) & (\bmod p), \\
\left(\tilde{a}_{2} x+\tilde{b}_{2}, \tilde{a}_{2} y+\tilde{b}_{2}, \tilde{a}_{2} z+\tilde{b}_{2}\right) \equiv(0,1,0) & (\bmod p), \\
\left(\tilde{a}_{3} x+\tilde{b}_{3}, \tilde{a}_{3} y+\tilde{b}_{3}, \tilde{a}_{3} z+\tilde{b}_{3}\right) \equiv(0,0,1) & (\bmod p)
\end{array}
$$


must hold simultaneously. This is impossible since otherwise $(x, y, z)$ and $(1,1,1)$ would generate the $\mathbb{Z} / p \mathbb{Z}$-vector space $(\mathbb{Z} / p \mathbb{Z})^{3}$. That is why we conclude that the $R(G)_{p}$-module $\operatorname{rad}\left(R(G)_{p}^{\prime} / R(G)_{p}\right)$ is not cyclic, whence $R(G)$ has infinite representation type.

The assumption $|P| \geq p^{3}$ in Lemma 4.13 is necessary. It is easy to show that the character ring of $G=C_{3}^{2} \rtimes C_{4}$ where $C_{4}$ acts on $C_{3}^{2}$ as a subgroup of $\Gamma \mathrm{L}_{0}\left(3^{2}\right)$ on $\mathbb{F}_{3}^{2}$ is representation-finite. A further example is given by the character ring of $G=C_{7}^{2} \rtimes \mathrm{SL}(2,3)$ where $G$ is isomorphic to a subgroup of the sharply 2-transitive permutation group of degree 49 which is not isomorphic to $C_{7}^{2} \rtimes \Gamma \mathrm{L}_{0}\left(7^{2}\right)$.

Next, we want to prove a lemma similar to Lemma 4.8. Therefore, we only consider such groups $G$ where $N_{G}(P) / C_{G}(P)$ acts on an elementary-abelian Sylow $p$-subgroup $P$ of $G$ as a subgroup of $\Gamma L_{0}(P)$ on $\mathbb{F}_{p^{k}}$. If $G$ has exactly two $\mathbb{Q}$-classes of elements of order $p$ and $|P|=p^{k}$, then it is clear that $k$ must be even.

Lemma 4.14. Let $k \geq 2$ be even, $P$ be the additive group of $\mathbb{F}_{p^{k}}$, and $C$ be a subgroup of the multiplicative group of $\mathbb{F}_{p^{k}}$. Moreover, suppose

$$
G:=\left\{\left(\begin{array}{ll}
a & x \\
0 & 1
\end{array}\right): a \in C, x \in P\right\}
$$

has exactly two $\mathbb{Q}$-classes of elements of order $p$. Let $u \in G$ be an element of order $p$, and $\mathfrak{p}$ be the maximal ideal in $\mathcal{O}_{\mathbb{Q}\left(\mathrm{cl}_{G}(u)\right)}$ containing $p$. If $u$ is not conjugate to $u^{-1}$, then $\chi(1) \equiv \chi(u)\left(\bmod \mathfrak{p}^{k}\right)$ for any $\chi \in \operatorname{Irr}(G)$. On the other hand, if $u$ is conjugate to $u^{-1}$, then $\chi(1) \equiv \chi(u)\left(\bmod \mathfrak{p}^{k / 2}\right)$ for any $\chi \in \operatorname{Irr}(G)$.

Proof. At first, we assume that $u$ is not conjugate to $u^{-1}$. We set

$$
V:=\left\{\left(\begin{array}{ll}
1 & x \\
0 & 1
\end{array}\right): x \in P\right\} \cong P \text { and } H:=\left\{\left(\begin{array}{ll}
a & 0 \\
0 & 1
\end{array}\right): a \in C\right\} \cong C
$$

so that $G=V \rtimes H$. As in the proof of Lemma 4.8, we obtain

$$
n:=|C|=\frac{p^{k}-1}{2(p-1)} \cdot t \quad \text { with } \operatorname{gcd}(n, p-1) \mid t \leq p-1 .
$$

Since the elements of order $p$ in $G$ are nonreal, none of the matrices in $H$ has upper left entry -1 . Therefore, $|H|$ is odd. Since $\left(p^{k}-1\right) / 2(p-1)$ is even for $p \equiv 3$ $(\bmod 4)$, we conclude $p \equiv 1(\bmod 4)$.

As in Lemma 4.8, $G$ is a Frobenius group with kernel $V$ and complement $H$. The conjugacy classes of $G$ have the following representatives:

(1) $1_{G}$,

(2) $P_{1}, \ldots, P_{(p-1) / t}$ where each $P_{i}$ has the form $\left(\begin{array}{ll}1 & x \\ 0 & 1\end{array}\right)$ for some $x \in \mathbb{F}_{p}$, 
(3) $P_{(p-1) / t+1}, \ldots, P_{2(p-1) / t}$ where each $P_{i}$ has the form $\left(\begin{array}{ll}1 & x \\ 0 & 1\end{array}\right)$ for some $x \notin$ $\mathbb{F}_{p}$,

(4) $C_{1}, \ldots, C_{\left(p^{k}-1\right) t / 2(p-1)}$ where each $C_{j}$ has the form $\left(\begin{array}{ll}a & 0 \\ 0 & 1\end{array}\right)$ with $a \in C$.

Again, the linear characters of $G$ are extensions of the linear characters of $H$. For a linear character $\chi \in \operatorname{Irr}(G)$, there exists a linear character $\lambda \in \operatorname{Irr}(H)$ such that $\chi(g)=\lambda(h)$ if $g=v h$ with $v \in V$ and $h \in H$. Hence, the assertion of the lemma is trivial for linear characters.

The nonlinear irreducible characters of $G$ can be gained by induction of the irreducible characters of $V$. Any irreducible character of $V$ is of the form $\tilde{\lambda}_{x}$ where $X:=\left(\begin{array}{ll}1 & x \\ 0 & 1\end{array}\right)$ lies in $\left\{P_{1}, \ldots, P_{2(p-1) / t}\right\}$ and $\tilde{\lambda}_{x}(Y)=\zeta_{p}^{\operatorname{Tr}(x y)}$ for $Y \in V$ with $Y=\left(\begin{array}{ll}1 & y \\ 0 & 1\end{array}\right)$. Obviously, we have $\tilde{\lambda}_{x}^{G}(1)=\left(p^{k}-1\right) t / 2(p-1)$ and $\tilde{\lambda}_{x}^{G}(g)=0$ if $g \notin V$. For $Y \in\left\{P_{1}, \ldots, P_{2(p-1) / t}\right\}$ with upper right entry $y$, we obtain

$$
\tilde{\lambda}_{x}^{G}(Y)=\frac{1}{|V|} \sum_{g \in G} \tilde{\lambda}_{x}^{\circ}\left(g Y g^{-1}\right)=\sum_{g \in H} \tilde{\lambda}_{x}\left(g Y g^{-1}\right)=\sum_{a \in C} \lambda_{x}(a y)=\sum_{a \in C} \zeta_{p}^{\operatorname{Tr}(x a y)} .
$$

We regard $\left(1-\zeta_{p}\right)$ as an ideal in $\mathcal{O}_{\mathbb{Q}\left(\zeta_{p}\right)}$. Then, we have to show

$$
\frac{p^{k}-1}{2(p-1)} \cdot t \equiv \sum_{a \in C} \zeta_{p}^{\operatorname{Tr}(a x y)} \quad\left(\bmod \left(1-\zeta_{p}\right)^{t k}\right) .
$$

For $i=1, \ldots,(p-1) / t$, all $P_{i}$ are contained in one cyclic group. Thus, each of the values $\tilde{\lambda}_{x}^{G}\left(P_{1}\right), \ldots, \tilde{\lambda}_{x}^{G}\left(P_{(p-1) / t}\right)$ can be transferred by Galois automorphisms of $\mathbb{Q}\left(\operatorname{cl}_{G}\left(P_{1}\right)\right) / \mathbb{Q}$ into each other and for these $P_{i}$, we only have to show $\left(p^{k}-\right.$ $1) t / 2(p-1) \equiv s_{x}\left(\bmod \left(1-\zeta_{p}\right)^{k t}\right)$ with $s_{x}=\sum_{a \in C} \zeta_{p}^{\operatorname{Tr}(a x)}$. Similarly, we can choose $P_{(p-1) / t+1}, \ldots, P_{2(p-1) / t}$ such that for $i=(p-1) / t+1, \ldots, 2(p-1) / t$, all $P_{i}$ are contained in a certain cyclic group. Hence, if $z$ is the upper right entry of $P_{2(p-1) / t}$, then for these $P_{i}$, it suffices to show $\left(p^{k}-1\right) t / 2(p-1) \equiv$ $s_{x z}\left(\bmod \left(1-\zeta_{p}\right)^{k t}\right)$ with $s_{x z}=\sum_{a \in C} \zeta_{p}^{\operatorname{Tr}(a x z)}$.

Let $M \subseteq \mathbb{F}_{p^{k}}^{\times}$be a transversal for the cosets $\mathbb{F}_{p^{k}}^{\times} / C$. We can regard $M$ as a subset of $\mathbb{F}_{p^{k}}^{\times}$as well as a group. For $m \in M$, we define

$$
s(m):=\sum_{a \in m^{-1} C} \zeta_{p}^{\operatorname{Tr}(a)}=\sum_{a \in C} \zeta_{p}^{\operatorname{Tr}(m a)} .
$$

Moreover, let $N \leq \operatorname{Irr}\left(\mathbb{F}_{p^{k}}^{\times}\right)$be the subgroup of the irreducible characters of $\mathbb{F}_{p^{k}}^{\times}$ which are trivial on $C$, so $|N|=2(p-1) / t$. For $\psi \in N$, we have the Gaussian sum

$$
\Gamma(\psi):=\sum_{\alpha \in \mathbb{F}_{p^{k}}^{\times}} \psi(\alpha) \zeta_{p}^{\operatorname{Tr}(\alpha)}=\sum_{m \in M} \sum_{a \in C} \psi(m a) \zeta_{p}^{\operatorname{Tr}(m a)}=\sum_{m \in M} \psi(m) s(m) .
$$


Since $N$ contains exactly the characters of $\mathbb{F}_{p^{k}}^{\times}$which correspond canonically to the irreducible characters of $\mathbb{F}_{p^{k}}^{\times} / C$, we can regard $N$ as the set of irreducible characters of $M$. With this approach, the function $\widetilde{\Gamma}$ given by $\widetilde{\Gamma}(\psi):=\Gamma(\bar{\psi})$ is the Fourier transform of $s$, whence

$$
s_{x}=s(x)=\frac{t}{2(p-1)} \sum_{\psi \in N} \overline{\psi(x)} \Gamma(\psi) \text { and } s_{x z}=s(x z)=\frac{t}{2(p-1)} \sum_{\psi \in N} \overline{\psi(x z)} \Gamma(\psi) .
$$

We only consider characters $\psi$ which are trivial on $C$, so $\psi(\alpha)=\alpha^{-\ell}$ for $\alpha \in \mathbb{F}_{p^{k}}^{\times}$ and some

$$
\ell \in\left\{\frac{p^{k}-1}{2(p-1)} \cdot t \cdot r: r \in\left\{0,1, \ldots, \frac{2(p-1)}{t}-1\right\}\right\} .
$$

For the trivial character, we get

$$
\overline{\mathbb{1}(x)} \Gamma(\mathbb{1})=\overline{\mathbb{1}(x z)} \Gamma(\mathbb{1})=\sum_{a \in \mathbb{F}_{p^{k}}^{\times}} \zeta_{p}^{\operatorname{Tr}(a)}=-1+\sum_{a \in \mathbb{F}_{p^{k}}} \zeta_{p}^{\operatorname{Tr}(a)}=-1
$$

If $\psi \in N$ is a nontrivial character, then we obtain

$$
\Gamma(\psi)=\Gamma_{\ell}=\sum_{\alpha \in \mathbb{F}_{p^{k}}^{\times}} \alpha^{-\ell} \zeta_{p}^{\operatorname{Tr}(\alpha)}
$$

for a suitable

$$
\ell \in\left\{\frac{p^{k}-1}{2(p-1)} \cdot t \cdot r: r \in\left\{1,2, \ldots, \frac{2(p-1)}{t}-1\right\}\right\} .
$$

If $r$ is even, then $\ell$ has the form $\ell=\left(p^{k}-1\right) t b /(p-1)=t b\left(1+\ldots+p^{k-1}\right)$ for some $b \in\{1,2, \ldots,(p-1) / t-1\}$. Each of these $b$ satisfies $t b<p$, whence the coefficients of the $p$-adic representation of $\ell$ can be read off this decomposition at once. Consequently, the sum of these coefficients equals $k t b$ and is minimal for $b=1$.

If $r$ is odd, then $\ell=\left(p^{k}-1\right) t(2 b+1) / 2(p-1)$ for some $b \in\{0,1, \ldots,(p-1) / t-1\}$. Moreover, $t$ is odd, i.e. $t=2 w+1$ for some integer $w \geq 0$. This yields

$$
\begin{aligned}
\ell & =\frac{p^{k}-1}{2(p-1)} \cdot(2 w+1) \cdot(2 b+1)=(2 w+1)(2 b+1) \cdot \frac{1+\ldots+p^{k-1}}{2} \\
& =(4 b w+2(b+w)+1) \cdot \frac{p+1}{2} \cdot\left(1+p^{2}+\ldots+p^{k-2}\right) \\
& =\left((2 b w+b+w)(p+1)+\frac{p+1}{2}\right) \cdot\left(1+p^{2}+\ldots+p^{k-2}\right) \\
& =\left(2 b w+b+w+\frac{p+1}{2}+(2 b w+b+w) p\right) \cdot\left(1+p^{2}+\ldots+p^{k-2}\right) .
\end{aligned}
$$


Since $(2 w+1)(2 b+1)=t r \leq 2(p-1)-t<2(p-1)$, we obtain

$$
\begin{aligned}
2 b w+b+w & =\frac{4 b w+2(b+w)}{2} \\
& <\frac{4 b w+2(b+w)+1}{2}=\frac{(2 w+1)(2 b+1)}{2}=\frac{t r}{2}<p-1,
\end{aligned}
$$

so $2 b w+b+w+(p+1) / 2<2 p$. In the case $2 b w+b+w+(p+1) / 2<p$, the coefficients of the $p$-adic representation of $\ell$ can be directly read off the decomposition above. For $2 b w+b+w+(p+1) / 2 \geq p$, we get

$$
\ell=(c+(2 b w+b+w+1) p) \cdot\left(1+p^{2}+\ldots+p^{k-2}\right)
$$

for some $c<p$. Then, the coefficients of the $p$-adic representation of $\ell$ can be read off this equation. In the first case, the coefficients of the odd powers of $p$ are $\geq(p+1) / 2$, in the second case, this holds for the coefficients of the even powers of $p$. Thus, in both cases, the sum of the coefficients is at least

$$
\frac{k}{2} \cdot \frac{p+1}{2}=\frac{k(p+1)}{4} .
$$

Since $p \equiv 1(\bmod 4)$ and $t$ is an odd divisor of $p-1, t$ also divides $(p-1) / 4$. This implies $k(p+1) / 4>k t$. Therefore, Theorem 4.7 yields $\Gamma_{\ell} \equiv 0\left(\bmod \left(1-\zeta_{p}\right)^{k t+1}\right)$ in $\mathbb{Z}_{(p)}\left[\zeta_{p}\right]$ for

$$
\ell \in\left\{\frac{p^{k}-1}{p-1} \cdot t \cdot r: r \in\left\{1,3,4, \ldots, \frac{2(p-1)}{t}-1\right\}\right\} .
$$

Moreover, for $r=2$, we get the congruence equation

$$
\Gamma_{\ell} \equiv-\frac{\left(1-\zeta_{p}\right)^{k t}}{(t !)^{k}} \quad\left(\bmod \left(1-\zeta_{p}\right)^{k t+1}\right)
$$

in $\mathbb{Z}_{(p)}\left[\zeta_{p}\right]$. This gives

$$
\begin{aligned}
s_{x}-\frac{p^{k}-1}{2(p-1)} \cdot t & =\frac{t}{2(p-1)} \sum_{\psi \in N} \overline{\psi(x)} \Gamma(\psi)-\frac{p^{k}-1}{2(p-1)} \cdot t \\
& \equiv \frac{t}{2(p-1)}\left(-1-\zeta_{2(p-1) / t}^{d} \cdot \frac{\left(1-\zeta_{p}\right)^{k t}}{(t !)^{k}}-p^{k}+1\right) \\
& \equiv-\frac{t}{2(p-1)} \cdot\left(\zeta_{2(p-1) / t}^{d} \cdot \frac{\left(1-\zeta_{p}\right)^{t k}}{(t !)^{k}}+p^{k}\right) \quad\left(\bmod \left(1-\zeta_{p}\right)^{k t+1}\right)
\end{aligned}
$$

for some $d \in \mathbb{Z}$ such that $\overline{\psi(x)}=\zeta_{2(p-1) / t}^{d}$. Analogously, we get

$s_{x z}-\frac{p^{k}-1}{2(p-1)} \cdot t \equiv-\frac{t}{2(p-1)} \cdot\left(\zeta_{2(p-1) / t}^{d} \cdot \frac{\left(1-\zeta_{p}\right)^{t k}}{(t !)^{k}}+p^{k}\right) \quad\left(\bmod \left(1-\zeta_{p}\right)^{k t+1}\right)$

if we choose $d \in \mathbb{Z}$ such that $\overline{\psi(x z)}=\zeta_{2(p-1) / t}^{d}$. In particular,

$$
s_{x}-\frac{p^{k}-1}{2(p-1)} \cdot t \quad \text { and } \quad s_{x z}-\frac{p^{k}-1}{2(p-1)} \cdot t
$$


are contained in $\left(\left(1-\zeta_{p}\right)^{t k}\right)$. This proves the first assertion of the lemma.

The case that $u$ and $u^{-1}$ are conjugate in $G$ can be dealt with analogously. The only difference is that in this case, $t$ is even, i.e. $t=2 w$ for some positive integer $w$. With the same notations as above, we obtain

$$
\Gamma(\psi)=\Gamma_{\ell}=\sum_{\alpha \in \mathbb{F}_{p^{k}}^{\times}} \alpha^{-\ell} \zeta_{p}^{\operatorname{Tr}(\alpha)}
$$

for a suitable

$$
\ell \in\left\{\frac{p^{k}-1}{2(p-1)} \cdot t \cdot r: r \in\left\{1,2, \ldots, \frac{2(p-1)}{t}-1\right\}\right\} .
$$

Since $t=2 w$, this yields $\ell=w r\left(1+\ldots+p^{k-1}\right)$ for some $r \in\{1,2, \ldots,(p-1) / w-1\}$. This gives $\Gamma_{\ell} \equiv 0\left(\bmod \left(1-\zeta_{p}\right)^{1+k t / 2}\right)$ for

$$
\ell \in\left\{\frac{p^{k}-1}{2(p-1)} \cdot t \cdot r: r \in\left\{2,3, \ldots, \frac{2(p-1)}{t}-1\right\}\right\}
$$

as well as $\Gamma_{\ell} \equiv-\left(1-\zeta_{p}\right)^{k t / 2} /(w !)^{k}\left(\bmod \left(1-\zeta_{p}\right)^{1+k t / 2}\right)$ for $r=1$. The remaining part of the proof is analogous to the first case.

Proposition 4.15. Let $P \in \operatorname{Syl}_{p}(G)$ be elementary-abelian of order $p^{k} \geq p^{2}, G$ contain exactly two $\mathbb{Q}$-classes of elements of order $p$, and $N_{G}(P) / C_{G}(P)$ act on $P$ as a subgroup of $\Gamma \mathrm{L}\left(p^{k}\right)$ on $\mathbb{F}_{p}^{k}$.

(1) If $k>2$, then $R(G)$ has infinite representation type.

(2) If $k=2$ and $\left|N_{G}(P) / C_{G}(P)\right|$ is odd, then $R(G)$ has infinite representation type.

Proof. Applying Lemma 4.14, the proof of this proposition is analogous to the proof of Theorem 4.9. We only remark that for $k=2$ and $\left|N_{G}(P) / C_{G}(P)\right|$ odd, the radical $\operatorname{rad}\left(R(G)_{p}^{\prime} / R(G)_{p}\right)$ is also not cyclic. Let $x, y \in P$ be representatives of the $\mathbb{Q}$-classes of $G$ containing elements of order $p$. The maximal ideal $\mathfrak{P}$ of $\mathbb{Z}_{(p)} \otimes$ $\mathcal{O}_{\mathbb{Q}\left(\operatorname{cl}_{G}(x)\right)}$ coincides with the maximal ideal of $\mathbb{Z}_{(p)} \otimes \mathcal{O}_{\mathbb{Q}\left(\operatorname{cl}_{G}(y)\right)}$. By Lemma 4.14, there is no function $\alpha \in \operatorname{rad}\left(R(G)_{p}^{\prime}\right)$ such that there exist $\eta_{1}, \eta_{2}, \xi_{1}, \xi_{2} \in R(G)_{p}$ satisfying $\eta_{1}(x) \alpha(x)+\xi_{1}(x) \in \mathfrak{P} \backslash \mathfrak{P}^{2}, \eta_{1}(y) \alpha(y)+\xi_{1}(y)=0, \eta_{2}(x) \alpha(x)+\xi_{2}(x)=0$, and $\eta_{2}(y) \alpha(y)+\xi_{2}(y) \in \mathfrak{P} \backslash \mathfrak{P}^{2}$. Consequently, the $R(G)_{p}$-module $\operatorname{rad}\left(R(G)_{p}^{\prime} / R(G)_{p}\right)$ is not cyclic.

The assumption in Proposition 4.15 that $\left|N_{G}(P) / C_{G}(P)\right|$ is odd for $k=2$ is necessary as the following example shows.

Example 4.16. Let $P \cong C_{5}^{2}, C \cong C_{6}$, and $G=P \rtimes C$ where $C$ acts on $P$ as a subgroup of $\Gamma \mathrm{L}_{0}\left(5^{2}\right)$ on $\mathbb{F}_{5}^{2}$. For an element $x \in G$ of order 5 , it is easy to show that $(1+\sqrt{5}) / 2$ lies in $\mathbb{Z}[\chi(x): \chi \in \operatorname{Irr}(G)]$, so $\mathbb{Z}[\chi(x): \chi \in \operatorname{Irr}(G)]$ coincides 
with $\mathcal{O}_{\mathbb{Q}\left(\mathrm{cl}_{G}(x)\right)}=\mathcal{O}_{\mathbb{Q}(\sqrt{5})}$. Since $G$ has exactly two $\mathbb{Q}$-classes of elements of order 5 , the functions $\alpha_{1}, \alpha_{2} \in R(G)_{5}^{\prime}$ with $\alpha_{1}(1)=1, \alpha_{1}(g)=0$ for $1 \neq g \in G$, and $\alpha_{2}(x)=1, \alpha_{2}(g)=0$ for $g \in G \backslash \operatorname{cl}_{G}(x)$ generate the $R(G)_{5}$-module $R(G)_{5}^{\prime} / R(G)_{5}$. Moreover, we can find $y \in G \backslash \operatorname{cl}_{G}(x)$ of order 5 such that the function $\varphi$ with $\varphi(x)=2 \sqrt{5}, \varphi(y)=\sqrt{5}$, and $\varphi(g)=0$ for $g \in G \backslash P$ is contained in $R(G)$. Since $\operatorname{rad}\left(\mathbb{Z}_{(5)} \otimes \mathcal{O}_{\mathbb{Q}(\sqrt{5})}\right)=(\sqrt{5})$, the function $\beta$ which is given by $\beta(x)=\sqrt{5}$ and $\beta(g)=0$ for $g \in G \backslash \operatorname{cl}_{G}(x)$ generates the $R(G)_{5}$-module $\operatorname{rad}\left(R(G)_{5}^{\prime} / R(G)_{5}\right)$. Finally, for $p \in\{2,3\}$, the Sylow $p$-subgroups of $G$ are cyclic of order $p$ and $C$ is a self-normalizing TI-subgroup of $G$, whence $R(G)$ is representation-finite.

Remark 4.17. Foulser [19] and Dornhoff [14] have classified the solvable primitive permutation groups of rank 3. These groups are listed in [19, Theorem 1.1] and are partitioned in three classes there. The first class consists of the subgroups of the semilinear groups. The second class contains the finite set of remaining groups which are also vector space primitive and the third class contains the groups which are vector space imprimitive. If $G$ is a group in the third class acting on a respective vector space $V$, then the orbits of $G$ on $V \backslash\{0\}$ are $\left(V_{1} \cup V_{2}\right) \backslash\{0\}$ and $V \backslash\left(V_{1} \cup V_{2}\right)$ for suitable subspaces $V_{1}, V_{2}$ of $V$ with $V=V_{1} \oplus V_{2}$. Moreover, $\operatorname{stab}_{G}\left(V_{i}\right)$ acts transitively on $V_{i}$ for $i=1,2$.

Let $G$ be solvable with elementary-abelian p-subgroup $P$ of order $p^{k} \geq p^{2}$ such that $G$ has exactly two $\mathbb{Q}$-classes of elements of order $p$. If $N_{G}(P) / C_{G}(P)$ acts on $P$ as a subgroup of a group in the first class, then Proposition 4.15 is applicable. Moreover, if $N_{G}(P) / C_{G}(P)$ acts on $P$ as a subgroup of a group in the second class and $R(G)$ is representation-finite, then it is not difficult to show that $P \rtimes N_{G}(P) / C_{G}(P)$ is isomorphic to $C_{7}^{2} \rtimes \mathrm{SL}(2,3)$ or $C_{23} \rtimes\left(C_{11} \times \mathrm{SL}(2,3)\right)$ where both of these groups are subgroups of exceptional doubly transitive groups. However, if $N_{G}(P) / C_{G}(P)$ acts on $P$ as a subgroup of a group in the third class, it seems to be difficult to answer the question whether $R(G)$ is representation-finite in general.

Example 4.18. Let $P \cong C_{7}^{2}, H \cong C_{9} \rtimes C_{4}$ be nonabelian, and $G=P \rtimes H$ where $H$ acts as a subgroup of a group in the third class of the theorem of Foulser such that $G$ has exactly two $\mathbb{Q}$-classes of elements of order 7 ( $G$ coincides with the group SmallGroup $(1764,64)$ in GAP). Then, one of these $\mathbb{Q}$-classes is already a conjugacy class whereas the other $\mathbb{Q}$-class, say $\mathcal{C}$, splits into three conjugacy classes. Let $\mathfrak{p}$ be the maximal ideal in $\mathcal{O}_{\mathbb{Q}(\mathcal{C})}$ containing 7 . Then, for all $x \in \mathcal{C}$ and all $\chi \in$ $\operatorname{Irr}(G)$, we have $\chi(1) \equiv \chi(x)\left(\bmod \mathfrak{p}^{2}\right)$. Thus, $R(G)_{7}$ has infinite representation type. We remark that the elements of order 7 are real in $G$. 
If $P \in \operatorname{Syl}_{p}(G)$ is an elementary-abelian group of order $p^{k} \geq p^{2}, N_{G}(P) / C_{G}(P)$ does not act on $P$ as a subgroup of $\Gamma \mathrm{L}\left(p^{k}\right)$ on $\mathbb{F}_{p}^{k}$, and $G$ has exactly two $\mathbb{Q}$ classes of elements of order $p$, then several examples suggest that $R(G)$ can only be representation-finite if $k=2$ and both of the $\mathbb{Q}$-classes of elements of order $p$ of $G$ are already conjugacy classes. However, this is merely a conjecture.

Example 4.19. We consider the group $G=C_{11}^{2} \rtimes Q_{12}$ where $Q_{12}$ is the dicyclic group of order 12 and $Q_{12}$ acts on $C_{11}^{2}$ such that $G$ has exactly two $\mathbb{Q}$-classes of elements of order 11 . For any $x \in G$ of order 11 and any $\chi \in \operatorname{Irr}(G)$, the congruence $\chi(1) \equiv \chi(x)\left(\bmod \mathfrak{p}^{2}\right)$ holds where $\mathfrak{p}$ is the maximal ideal in $\mathcal{O}_{\mathbb{Q}\left(\operatorname{cl}_{G}(x)\right)}$ containing 11. Thus, $R(G)_{11}$ has infinite representation type although the elements of order 11 are real in $G$.

On the other hand, the character ring of $G=C_{11}^{2} \rtimes\left(C_{5} \times Q_{12}\right)$ where $C_{5}$ acts nontrivially on $C_{11}^{2}$ and $Q_{12}$ acts on $C_{11}^{2}$ as above has finite representation type. This follows immediately from the structure of the character table of $G$.

A further example is given by the group $G=C_{7}^{2} \rtimes \mathrm{SL}(2,3)$ which has only two $\mathbb{Q}$-classes of elements of order 7 , but is no subgroup of a 2-transitive group. For a nonrational element $x \in G$ of order 7 , we get $\chi(1) \equiv \chi(x)\left(\bmod \mathfrak{p}^{2}\right)$ if $\chi \in \operatorname{Irr}(G)$ and $\mathfrak{p}$ denotes the maximal ideal in $\mathcal{O}_{\mathbb{Q}\left(\operatorname{cl}_{G}(x)\right)}$ containing 7 . Hence, $R(G)_{7}$ has infinite representation type.

There are several other examples for groups $G$ with an elementary-abelian Sylow $p$-subgroup $P$ of order $\geq p^{2}$ such that $G$ has exactly two $\mathbb{Q}$-classes of elements of order $p$ and at least one of these $\mathbb{Q}$-classes is no conjugacy class. The respective actions of $N_{G}(P) / C_{G}(P)$ on $P$ differ, but in any example the author has considered, $R(G)$ has infinite representation type.

4.3. General assertions. Now, we want to combine the results of the previous sections. Prior to this, we give a proposition which is independent of the structure of the Sylow subgroups of $G$.

Proposition 4.20. Let $G_{1}, G_{2}$ be finite groups with $\operatorname{gcd}\left(\left|G_{1}\right|,\left|G_{2}\right|\right)=1$. Then, $R\left(G_{1} \times G_{2}\right)$ is representation-finite if and only if $R\left(G_{1}\right)$ and $R\left(G_{2}\right)$ have finite representation type.

Proof. It is clear that $R\left(G_{1} \times G_{2}\right)$ has infinite representation type if one of $R\left(G_{1}\right)$ and $R\left(G_{2}\right)$ is not representation-finite. That is why we assume that $R\left(G_{1}\right)$ and $R\left(G_{2}\right)$ are representation-finite.

Let $x_{1} \in G_{1}$ and $x_{2} \in G_{2}$, and let $A_{1}, \ldots, A_{m}$ and $B_{1}, \ldots, B_{n}$ be the conjugacy classes of $G_{1}$ and $G_{2}$ respectively. Then, the conjugacy classes of $G_{1} \times G_{2}$ are $A_{i} B_{j}$ with $i=1, \ldots, m$ and $j=1, \ldots, n$. Hence, for some prime divisor $p_{1}$ of $\left|G_{1}\right|$, the 
rational $p_{1}^{\prime}$-section of $\left(x_{1}, 1_{G_{2}}\right)$ in $G_{1} \times G_{2}$ has exactly as many $\mathbb{Q}$-classes as the rational $p_{1}^{\prime}$-section of $x_{1}$ in $G_{1}$. If $p_{2}$ is a prime divisor of $\left|G_{2}\right|$, then the rational $p_{2}^{\prime}$-section of $\left(x_{1}, 1_{G_{2}}\right)$ in $G_{1} \times G_{2}$ contains exactly as many $\mathbb{Q}$-classes as the rational $p_{2}^{\prime}$-section of $1_{G_{2}}$ in $G_{2}$. Similar assertions hold for $x_{2}$.

Let $\mathcal{C}$ be the $\mathbb{Q}$-class of $\left(x_{1}, x_{2}\right)$ in $G_{1} \times G_{2}$, and $\mathcal{C}_{i}$ be the $\mathbb{Q}$-class of $x_{i}$ in $G_{i}$, $i=1,2$. As is well known, the irreducible characters of $G_{1} \times G_{2}$ are the characters $\psi \times \varphi$ with $\psi \in \operatorname{Irr}\left(G_{1}\right)$ and $\varphi \in \operatorname{Irr}\left(G_{2}\right)$. Therefore, any class function of $G_{1} \times G_{2}$ is a product of a class function of $G_{1}$ with a class function of $G_{2}$.

Obviously, we have $\mathbb{Q}\left(\mathcal{C}_{1}\right) \subseteq \mathbb{Q}\left(\zeta_{\left|G_{1}\right|}\right)$ and $\mathbb{Q}\left(\mathcal{C}_{2}\right) \subseteq \mathbb{Q}\left(\zeta_{\left|G_{2}\right|}\right)$. Since the discriminants of $\mathbb{Q}\left(\zeta_{\left|G_{1}\right|}\right)$ and $\mathbb{Q}\left(\zeta_{\left|G_{2}\right|}\right)$ are coprime, this yields

$$
\mathcal{O}_{\mathbb{Q}(\mathcal{C})}=\mathcal{O}_{\mathbb{Q}\left(\mathcal{C}_{1}\right)} \mathcal{O}_{\mathbb{Q}\left(\mathcal{C}_{2}\right)}
$$

Let $p$ be a prime with $p|| G_{1} \mid, \mathcal{S}_{1}$ be the rational $p^{\prime}$-section of $x_{1}$ in $G_{1}$, and $\mathcal{S}$ be the rational $p^{\prime}$-section of $\left(x_{1}, x_{2}\right)$ in $G_{1} \times G_{2}$. Since $\left|G_{1}\right|$ is coprime to $\left|G_{2}\right|$, the function $\nu_{x_{2}}$ of $G_{2}$ with $\nu_{x_{2}}\left(x_{2}\right)=1$ and $\nu_{x_{2}}(g)=0$ for $g \in G_{2} \backslash \mathcal{C}_{2}$ lies in $R\left(G_{2}\right)_{p}$ by Lemma 2.7. Due to this and (6), if $\alpha_{1}$ is a generator of the $R\left(G_{1}\right)_{p}$-module $\left(R\left(G_{1}\right)_{p}^{\prime} \cap \mathrm{Ch}_{\mathbb{Q}}\left(\mathcal{S}_{1}\right)\right) /\left(R\left(G_{1}\right)_{p} \cap \mathrm{Ch}_{\mathbb{Q}}\left(\mathcal{S}_{1}\right)\right)$, then $\alpha_{1} \times \nu_{x_{2}}$ generates the $R\left(G_{1} \times G_{2}\right)_{p^{-}}$ module

$$
\left(R\left(G_{1} \times G_{2}\right)_{p}^{\prime} \cap \mathrm{Ch}_{\mathbb{Q}}(\mathcal{S})\right) /\left(R\left(G_{1} \times G_{2}\right)_{p} \cap \mathrm{Ch}_{\mathbb{Q}}(\mathcal{S})\right) .
$$

The same assertion for $\alpha_{1} \times \nu_{x_{2}}$ and $\alpha_{2} \times \nu_{x_{2}}$ holds if $\alpha_{1}$ and $\alpha_{2}$ generate the $R\left(G_{1}\right)_{p}$-module $\left(R\left(G_{1}\right)_{p}^{\prime} \cap \mathrm{Ch}_{\mathbb{Q}}\left(\mathcal{S}_{1}\right)\right) /\left(R\left(G_{1}\right)_{p} \cap \mathrm{Ch}_{\mathbb{Q}}\left(\mathcal{S}_{1}\right)\right)$.

We can argue analogously to show that the $R\left(G_{1} \times G_{2}\right)_{p}$-module

$$
\operatorname{rad}\left(\left(R\left(G_{1} \times G_{2}\right)_{p}^{\prime} \cap \mathrm{Ch}_{\mathbb{Q}}(\mathcal{S})\right) /\left(R\left(G_{1} \times G_{2}\right)_{p} \cap \mathrm{Ch}_{\mathbb{Q}}(\mathcal{S})\right)\right)
$$

is cyclic if the $R\left(G_{1}\right)_{p}$-module $\operatorname{rad}\left(\left(R\left(G_{1}\right)_{p}^{\prime} \cap \mathrm{Ch}_{\mathbb{Q}}\left(\mathcal{S}_{1}\right)\right) /\left(R\left(G_{1}\right)_{p} \cap \mathrm{Ch}_{\mathbb{Q}}\left(\mathcal{S}_{1}\right)\right)\right)$ is cyclic. Since $R\left(G_{1}\right)$ is representation-finite, we conclude that $R\left(G_{1} \times G_{2}\right)_{p}$ has also finite representation type.

The same arguments show that for a prime divisor $\ell$ of $\left|G_{2}\right|, R\left(G_{1} \times G_{2}\right)_{\ell}$ is representation-finite. Thus, $R\left(G_{1} \times G_{2}\right)$ has finite representation type.

Remark 4.21. If some prime $p$ divides $\operatorname{gcd}\left(\left|G_{1}\right|,\left|G_{2}\right|\right)$, then there are at least three $\mathbb{Q}$-classes of elements of order $p$ in $G_{1} \times G_{2}$. That is why $R\left(G_{1} \times G_{2}\right)$ has infinite representation type in this case.

Theorem 4.22. Let $G$ be a group of odd order. Then, the following are equivalent:

(1) $R(G)$ has finite representation type,

(2) $|G|$ is cube-free and all Sylow subgroups of $G$ are cyclic.

Proof. Theorem 4.6 yields the implication $(2) \Rightarrow(1)$ at once. Thus, we assume that $R(G)$ is representation-finite. Suppose $G$ has a (noncyclic) elementary-abelian 
Sylow $p$-subgroup $P$ for some prime $p$. Since $|G|$ is odd, the nontrivial elements of $P$ are nonreal. By Corollary 4.10 and Proposition $4.11, G$ has exactly two $\mathbb{Q}$ classes of elements of order $p$. Now, $G$ is solvable, so $N_{G}(P) / C_{G}(P)$ acts on $P$ as a subgroup of a group listed in the classification theorem of Foulser and Dornhoff (see Remark 4.17). Since $|G|$ is odd, $N_{G}(P) / C_{G}(P)$ cannot act on $P$ as a subgroup of a group in the second class. Moreover, if $N_{G}(P) / C_{G}(P)$ acted on $P$ as a subgroup of a group in the first class, then $R(G)$ would have infinite representation type by Proposition 4.15 .

Suppose $N_{G}(P) / C_{G}(P)$ acts on $P$ as a subgroup $H$ of a group in the third class on a vector space $V$ with $|V|=p^{k}$. Then, we get the decomposition $V=V_{1} \oplus V_{2}$ for suitable subspaces $V_{1}, V_{2}$ of $V$ such that $H$ acts on $\left(V_{1} \cup V_{2}\right) \backslash\{0\}$ as well as $V \backslash\left(V_{1} \cup V_{2}\right)$. Now, $|G|$ is odd whence $H$ acts on $V_{i}$ as a subgroup of $\Gamma \mathrm{L}\left(\left|V_{i}\right|\right)$ for $i=1,2$. Suppose $\left|V_{i}\right|=p^{\ell}$ for some $i \in\{1,2\}$ and some $\ell>1$, and let $Q<P$ be the subgroup corresponding to $V_{i}$. Then, for $x \in Q, \chi \in \operatorname{Irr}(G)$, and the maximal ideal $\mathfrak{p}$ of $\mathcal{O}_{\mathbb{Q}\left(\operatorname{cl}_{G}(x)\right)}$ containing $p$, we obtain $\chi(1) \equiv \chi(x)\left(\bmod \mathfrak{p}^{\ell}\right)$ as in Section 4.2.1. Since $x$ is nonreal, this yields that $R(G)$ would have infinite representation type.

That is why $|P|=p^{2}$ and the orbits of the action of $N_{G}(P) / C_{G}(P)$ on $P \backslash\{1\}$ have size $2(p-1)$ and $p^{2}-1-2(p-1)=(p-1)^{2}$. But this means that $N_{G}(P) / C_{G}(P)$ permutes transitively the cyclic groups of order $p$ corresponding to $V_{1}$ and $V_{2}$. Clearly, this is impossible because $|G|$ is odd.

Theorem 4.22 and Proposition 4.20 give an algorithm for the determination of the representation type of $R(G)$. If $|G|$ is odd, then we apply Theorem 4.22. Otherwise, we write $G=G_{1} \times G_{2}$ for subgroups $G_{1}, G_{2} \leq G$ such that $\left|G_{1}\right|$ is even and there are no subgroups $H, K$ of $G_{1}$ with $G_{1}=H \times K$. Then, $R(G)$ is representation-finite if and only if $\operatorname{gcd}\left(G_{1}, G_{2}\right)=1$ and $R\left(G_{1}\right)$ as well as $R\left(G_{2}\right)$ are representation-finite. Thus, the crucial part is to determine the representation type of $R\left(G_{1}\right)$.

In the previous sections, we have mainly considered the abelian Sylow $p$-subgroups of such a group $G_{1}$. One may also ask for properties $G_{1}$ must have if $G_{1}$ has a nonabelian Sylow 2-subgroup $P$ and $R\left(G_{1}\right)_{2}$ is representation-finite. With respect to the action of $N_{G_{1}}(P)$ on $P$, the answer is easy to find since $G_{1}$ contains at most three $\mathbb{Q}$-classes of 2 -elements. Thus, the examples following Theorem 3.17 yield minimal groups $G$ such that the Sylow 2-subgroups of $G$ are in the list of this theorem and $R(G)$ is representation-finite. That is why $G_{1}$ contains one of these minimal groups if $P \neq D_{8}$ and $R\left(G_{1}\right)$ has finite representation type. For $P \cong D_{8}$, if $R\left(G_{1}\right)$ is representation-finite, then $G_{1}$ has a subgroup isomorphic to $\operatorname{PSL}(2, p)$ with $p \equiv \pm 7(\bmod 16)$ where $p \pm 1$ is not divisible by the third power of an odd prime. Altogether, we get the following result. 
Theorem 4.23. Let $G$ be a group of even order such that there exist no proper subgroups $G_{1}, G_{2}$ with $G=G_{1} \times G_{2}$. Then, $R(G)$ is representation-finite if for any prime divisor $p$ of $|G|$ and any $P \in \operatorname{Syl}_{p}(G)$, one of the following applies.

(1) $P$ is cyclic of order $p$ or $p^{2}$.

(2) $P \cong C_{p}^{k}$ for some odd integer $k \geq 3$ where $p^{k}-1$ is cube-free, all elements of order $p$ are conjugate in $G$, and $N_{G}(P) / C_{G}(P)$ has a cyclic subgroup of order $p^{k}-1$.

(3) $P \cong C_{p}^{2}$, G has exactly two $\mathbb{Q}$-classes of elements of order $p$, and

(i) both of the $\mathbb{Q}$-classes are also conjugacy classes, or

(ii) the elements of order $p$ are real, $p+1$ is not divisible by 16, and $N_{G}(P) / C_{G}(P)$ has a cyclic subgroup of order $(p+1) / 2$.

(4) $P \cong C_{p}^{2}, p \in\{2,5,11,29,59\}$, all elements of order $p$ are conjugate in $G$, and $N_{G}(P) / C_{G}(P)$ has a normal subgroup isomorphic to $C_{3}(p=2)$, $\mathrm{SL}(2,3)(p=5), C_{5} \times \mathrm{SL}(2,3)(p=11), \mathrm{SL}(2,5)(p=11), C_{7} \times \mathrm{SL}(2,5)$ $(p=29)$, or $C_{29} \times \mathrm{SL}(2,5)(p=59)$.

(5) $P \cong Q_{8}$ and $G$ has a subgroup isomorphic to $\mathrm{SL}(2,3)$.

(6) $P \cong D_{8}$ and $G$ has a subgroup isomorphic to $\operatorname{PSL}(2, q)$ for some $q \equiv \pm 7$ ( $\bmod 16)$ where $q \pm 1$ is not divisible by the third power of an odd prime.

(7) $P \cong C_{p}^{k}$ for some $k \geq 2, G$ has exactly two $\mathbb{Q}$-classes of elements of order $p$, at least one of these $\mathbb{Q}$-classes is no conjugacy class, and $N_{G}(P) / C_{G}(P)$ does not act on $P$ as a subgroup of $\Gamma \mathrm{L}\left(p^{k}\right)$ on $\mathbb{F}_{p}^{k}$.

We have seen that for each $i \in\{1, \ldots, 6\}$, there exists a group $G$ with a Sylow $p$-subgroup $P$ such that $G$ and $P$ satisfy all conditions of part $i$ in the theorem above and $R(G)$ has finite representation type. The author presumes that this does not hold for part 7, i.e. $R(G)$ has infinite representation type if $G$ and some $P \in \operatorname{Syl}_{p}(G)$ satisfy all conditions of part 7 of Theorem 4.23.

In Section 4.2.1, we have seen that it is not enough to consider only the rational $p^{\prime}$-section of $p$-elements of a group with elementary-abelian Sylow $p$-subgroups. Similar problems arise for the rational $2^{\prime}$-sections of a group $G$ as in Theorem 4.23 if the Sylow 2-subgroups of $G$ are noncyclic. For instance, let $G=\left(C_{2}^{2} \times C_{7}\right) \rtimes C_{3}$ where $C_{3}$ centralizes none of the elements of order 2 or 7 respectively. If $\mathcal{S}$ denotes the $\mathbb{Q}$-class of elements of order 14 , then a bit lengthy calculation shows that each generating system of the $R(G)_{2}$-module $\left(R(G)_{2}^{\prime} \cap \mathrm{Ch}_{\mathbb{Q}}(\mathcal{S})\right) /\left(R(G)_{2} \cap \mathrm{Ch}_{\mathbb{Q}}(\mathcal{S})\right)$ has at least four elements. We remark that the $R(G)_{2}$-module $\operatorname{rad}\left(R(G)_{2}^{\prime} / R(G)_{2}\right)$ is cyclic. Hence, $R(G)$ has infinite representation type, but the reason for this is not the same as the reason for $R\left(\left(C_{3}^{3} \times C_{5}\right) \rtimes C_{52}\right)$ having infinite representation type in Example 4.12. Analogously (considering the $\mathbb{Q}$-class of elements of order 28), we 
can show that $R\left(\left(Q_{8} \times C_{7}\right) \rtimes C_{3}\right)$ has infinite representation type where $C_{3}$ acts nontrivially on both $Q_{8}$ and $C_{7}$.

The groups $\left(C_{2}^{2} \times C_{7}\right) \rtimes C_{3},\left(Q_{8} \times C_{7}\right) \rtimes C_{3}$ have one property in common with the groups of Example 4.12. If $G$ is one of these four groups, there is some prime $p$ such that $G$ has a noncyclic Sylow $p$-subgroup $P$ satisfying $C_{G}\left(N_{G}(P)\right)<C_{G}(P)$. Possibly, this can be generalized as follows: if $G$ has an elementary-abelian Sylow p-subgroup $P$ or a Sylow 2-subgroup $P \neq D_{8}$ as in Theorem 3.17 and if there exists some $H \leq N_{G}(P)$ such that $H / P \cong N_{G}(P) / C_{G}(P)$ and $C_{G}(H)<C_{G}(P)$, then $R(G)$ has infinite representation type. However, this conjecture is based on only a few examples and these examples suggest that it will be hard to prove the conjecture (in case it holds). Surely, groups with Sylow 2-subgroups isomorphic to $D_{8}$ have to be considered separately since $D_{8}$ is not resistant.

Acknowledgment. The author thanks the referees for various valuable hints. Moreover, he is grateful to B. Külshammer for several useful suggestions and K. Haberland for the proof of Lemma 4.8. Finally, he gives thanks to E. Fritzsche for his useful comments. This paper summarizes the author's Ph.D. thesis [21]. 


\section{Appendix - character tables}

Apart from the character tables of $\operatorname{PSL}(2, q)$ and $\operatorname{SL}(2, q)$ which can be found in [15] and [32] respectively, the following character tables can be determined with GAP. The numbers in the first line of a table give the orders of the elements of a transversal for the conjugacy classes. Solely, the columns of the character tables of $\operatorname{PSL}(2, q)$ and $\operatorname{SL}(2, q)$ are indexed by elements.

\section{Quasisimple groups.}

- $A_{7}$

\begin{tabular}{lrrrrrrrc}
\hline & 1 & 2 & 31 & $3_{2}$ & 4 & 5 & 6 & $7_{ \pm}$ \\
\hline$\chi_{1}$ & 1 & 1 & 1 & 1 & 1 & 1 & 1 & 1 \\
$\chi_{2}$ & 6 & 2 & 0 & 3 & 0 & 1 & -1 & -1 \\
$\chi_{3,+}$ & 10 & -2 & 1 & 1 & 0 & 0 & 1 & $\frac{-1 \pm \sqrt{-7}}{2}$ \\
$\chi_{3,-}$ & 10 & -2 & 1 & 1 & 0 & 0 & 1 & $\frac{-1 \mp \sqrt{-7}}{2}$ \\
$\chi_{4}$ & 14 & 2 & 2 & -1 & 0 & -1 & 2 & 0 \\
$\chi_{5}$ & 14 & 2 & -1 & 2 & 0 & -1 & -1 & 0 \\
$\chi_{6}$ & 15 & -1 & 3 & 0 & -1 & 0 & -1 & 1 \\
$\chi_{7}$ & 21 & 1 & -3 & 0 & -1 & 1 & 1 & 0 \\
$\chi_{8}$ & 35 & -1 & -1 & -1 & 1 & 0 & -1 & 0 \\
\hline
\end{tabular}

- $\operatorname{PSL}(2, q)$ with $q \equiv 0(\bmod 2)$

\begin{tabular}{lcrcc}
\hline & 1 & 2 & $A^{j}$ & $B^{k}$ \\
\hline$\chi_{1}$ & 1 & 1 & 1 & 1 \\
$\chi_{2, \ell}$ & $q-1$ & -1 & 0 & $-\zeta_{q+1}^{k \ell}-\zeta_{q+1}^{-k \ell}$ \\
$\chi_{3}$ & $q$ & 0 & 1 & -1 \\
$\chi_{4, m}$ & $q+1$ & 1 & $\zeta_{q-1}^{j m}+\zeta_{q-1}^{-j m}$ & 0 \\
\hline
\end{tabular}

$|\langle A\rangle|=q-1, \quad|\langle B\rangle|=q+1$,

$j=1, \ldots, \frac{q-2}{2}, \quad k=1, \ldots, \frac{q}{2}, \quad \ell=1, \ldots, \frac{q}{2}, \quad m=1, \ldots, \frac{q-2}{2}$

- $\operatorname{PSL}(2, q)$ with $q \equiv 1(\bmod 4)$

\begin{tabular}{lccccc}
\hline & 1 & $p_{ \pm}$ & 2 & $A^{j}$ & $B^{k}$ \\
\hline$\chi_{1}$ & 1 & 1 & 1 & 1 & 1 \\
$\chi_{2,+}$ & $\frac{q+1}{2}$ & $\frac{1 \pm \sqrt{q}}{2}$ & $(-1)^{(q-1) / 4}$ & $(-1)^{j}$ & 0 \\
$\chi_{2,-}$ & $\frac{q+1}{2}$ & $\frac{1 \mp \sqrt{q}}{2}$ & $(-1)^{(q-1) / 4}$ & $(-1)^{j}$ & 0 \\
$\chi_{3, \ell}$ & $q-1$ & -1 & 0 & 0 & $-\zeta_{(q+1) / 2}^{k \ell}-\zeta_{(q+1) / 2}^{-k \ell}$ \\
$\chi_{4}$ & $q$ & 0 & 1 & 1 & -1 \\
$\chi_{5, m}$ & $q+1$ & 1 & $2 \cdot(-1)^{m}$ & $\zeta_{(q-1) / 2}^{j m}+\zeta_{(q-1) / 2}^{-j m}$ & 0 \\
\hline
\end{tabular}

$|\langle A\rangle|=\frac{q-1}{2}, \quad|\langle B\rangle|=\frac{q+1}{2}$,

$j=1, \ldots, \frac{q-5}{4}, \quad k=1, \ldots, \frac{q-1}{4}, \quad \ell=1, \ldots, \frac{q-1}{4}, \quad m=1, \ldots, \frac{q-5}{4}$ 
- $\operatorname{PSL}(2, q)$ with $q \equiv 3(\bmod 4)$

\begin{tabular}{lccccc}
\hline & 1 & $p_{ \pm}$ & 2 & $A^{j}$ & $B^{k}$ \\
\hline$\chi_{1}$ & 1 & 1 & 1 & 1 & 1 \\
$\chi_{2,+}$ & $\frac{q-1}{2}$ & $\frac{-1 \pm \sqrt{-q}}{2}$ & $(-1)^{(q-3) / 4}$ & 0 & $(-1)^{k+1}$ \\
$\chi_{2,-}$ & $\frac{q-1}{2}$ & $\frac{-1 \mp \sqrt{-q}}{2}$ & $(-1)^{(q-3) / 4}$ & 0 & $(-1)^{k+1}$ \\
$\chi_{3, \ell}$ & $q-1$ & -1 & $2 \cdot(-1)^{\ell+1}$ & 0 & $-\zeta_{(q+1) / 2}^{k \ell}-\zeta_{(q+1) / 2}^{-k \ell}$ \\
$\chi_{4}$ & $q$ & 0 & -1 & 1 & -1 \\
$\chi_{5, m}$ & $q+1$ & 1 & 0 & $\zeta_{(q-1) / 2}^{j m}+\zeta_{(q-1) / 2}^{-j m}$ & 0 \\
\hline
\end{tabular}

$|\langle A\rangle|=\frac{q-1}{2}, \quad|\langle B\rangle|=\frac{q+1}{2}$,

$j=1, \ldots, \frac{q-3}{4}, \quad k=1, \ldots, \frac{q-3}{4}, \quad \ell=1, \ldots, \frac{q-3}{4}, \quad m=1, \ldots, \frac{q-3}{4}$

- $\operatorname{SL}(2, q)$

\begin{tabular}{lcccccc}
\hline & 1 & 2 & $p_{ \pm}$ & $2 p_{ \pm}$ & $A^{j}$ & $B^{k}$ \\
\hline$\chi_{1}$ & 1 & 1 & 1 & 1 & 1 & 1 \\
$\chi_{2,+}$ & $\frac{q-\varepsilon}{2}$ & $-\frac{(q-\varepsilon)}{2}$ & $\frac{-\varepsilon \pm \sqrt{\varepsilon q}}{2}$ & $\frac{\varepsilon \mp \sqrt{\varepsilon q}}{2}$ & $\frac{(1-\varepsilon)(-1)^{j}}{2}$ & $\frac{(1+\varepsilon)(-1)^{k+1}}{2}$ \\
$\chi_{2,-}$ & $\frac{q-\varepsilon}{2}$ & $-\frac{(q-\varepsilon)}{2}$ & $\frac{-\varepsilon \mp \sqrt{\varepsilon q}}{2}$ & $\frac{\varepsilon \pm \sqrt{\varepsilon q}}{2}$ & $\frac{(1-\varepsilon)(-1)^{j}}{2}$ & $\frac{(1+\varepsilon)(-1)^{k+1}}{2}$ \\
$\chi_{3,+}$ & $\frac{q+\varepsilon}{2}$ & $\frac{(q+\varepsilon)}{2}$ & $\frac{\varepsilon \pm \sqrt{\varepsilon q}}{2}$ & $\frac{\varepsilon \pm \sqrt{\varepsilon q}}{2}$ & $\frac{(1+\varepsilon)(-1)^{j}}{2}$ & $\frac{(1-\varepsilon)(-1)^{k+1}}{2}$ \\
$\chi_{3,-}$ & $\frac{q+\varepsilon}{2}$ & $\frac{(q+\varepsilon)}{2}$ & $\frac{\varepsilon \mp \sqrt{\varepsilon q}}{2}$ & $\frac{\varepsilon \mp \sqrt{\varepsilon q}}{2}$ & $\frac{(1+\varepsilon)(-1)^{j}}{2}$ & $\frac{(1-\varepsilon)(-1)^{k+1}}{2}$ \\
$\chi_{4, \ell}$ & $q-1$ & $(-1)^{\ell}(q-1)$ & -1 & $(-1)^{\ell+1}$ & 0 & $-\zeta_{q+1}^{k \ell}-\zeta_{q+1}^{-k \ell}$ \\
$\chi_{5}$ & $q$ & $q$ & 0 & 0 & 1 & -1 \\
$\chi_{6, m}$ & $q+1$ & $(-1)^{m}(q+1)$ & 1 & $(-1)^{m}$ & $\zeta_{q-1}^{j m}+\zeta_{q-1}^{-j m}$ & 0 \\
\hline
\end{tabular}

$|\langle A\rangle|=q-1, \quad|\langle B\rangle|=q+1, \quad \varepsilon=(-1)^{(q-1) / 2}$, $j=1, \ldots, \frac{q-3}{2}, \quad k=1, \ldots, \frac{q-1}{2}, \quad \ell=1, \ldots, \frac{q-1}{2}, \quad m=1, \ldots, \frac{q-3}{2}$

\section{Subgroups of 2-transitive groups.}

- $C_{3}^{2} \rtimes C_{4}$

\begin{tabular}{rrccrr}
\hline & 1 & 2 & $4_{ \pm}$ & $3_{1}$ & $3_{2}$ \\
\hline$\chi_{1, k}$ & 1 & $(-1)^{k}$ & $( \pm i)^{k}$ & 1 & 1 \\
$\chi_{2,1}$ & 4 & 0 & 0 & 1 & -2 \\
$\chi_{2,2}$ & 4 & 0 & 0 & -2 & 1 \\
\hline
\end{tabular}

$k=1, \ldots, 4$

- $C_{5}^{2} \rtimes C_{6}$

\begin{tabular}{ccccccc}
\hline & 1 & 2 & $3_{j}$ & $6_{j}$ & $5_{1, \pm}$ & $5_{2, \pm}$ \\
\hline$\chi_{1, k}$ & 1 & $(-1)^{k}$ & $\zeta_{3}^{j k}$ & $\left(-\zeta_{3}^{j}\right)^{k}$ & 1 & 1 \\
$\chi_{2,+}$ & 6 & 0 & 0 & 0 & $1 \pm \sqrt{5}$ & $\frac{-3 \pm \sqrt{5}}{2}$ \\
$\chi_{2,-}$ & 6 & 0 & 0 & 0 & $1 \mp \sqrt{5}$ & $\frac{-3 \mp \sqrt{5}}{2}$ \\
$\chi_{3,+}$ & 6 & 0 & 0 & 0 & $\frac{-3 \pm \sqrt{5}}{2}$ & $1 \mp \sqrt{5}$ \\
$\chi_{3,-}$ & 6 & 0 & 0 & 0 & $\frac{-3 \mp \sqrt{5}}{2}$ & $1 \pm \sqrt{5}$ \\
\hline
\end{tabular}

$j=1,2, \quad k=1, \ldots, 6$ 
- $C_{7}^{2} \rtimes \mathrm{SL}(2,3)$

\begin{tabular}{lrrrrccc}
\hline & 1 & $7_{1}$ & $7_{2}$ & 2 & 4 & $3_{j}$ & $6_{j}$ \\
\hline$\chi_{1, k}$ & 1 & 1 & 1 & 1 & 1 & $\zeta_{3}^{j k}$ & $\zeta_{3}^{j k}$ \\
$\chi_{2, k}$ & 2 & 2 & 2 & -2 & 0 & $-\zeta_{3}^{j k}$ & $\zeta_{3}^{j k}$ \\
$\chi_{3}$ & 3 & 3 & 3 & 3 & -1 & 0 & 0 \\
$\chi_{4,1}$ & 24 & 3 & -4 & 0 & 0 & 0 & 0 \\
$\chi_{4,2}$ & 24 & -4 & 3 & 0 & 0 & 0 & 0 \\
\hline
\end{tabular}

$j=1,2, \quad k=1,2,3$

- $C_{19}^{2} \rtimes \mathrm{SL}(2,5)$

\begin{tabular}{lccrrrccc}
\hline & 1 & $19_{j}$ & 2 & 4 & 3 & 6 & $5_{ \pm}$ & $10_{ \pm}$ \\
\hline$\chi_{1}$ & 1 & 1 & 1 & 1 & 1 & 1 & 1 & 1 \\
$\chi_{2,+}$ & 2 & 2 & -2 & 0 & -1 & 1 & $\frac{-1 \pm \sqrt{5}}{2}$ & $\frac{1 \mp \sqrt{5}}{2}$ \\
$\chi_{2,-}$ & 2 & 2 & -2 & 0 & -1 & 1 & $\frac{-1 \mp \sqrt{5}}{2}$ & $\frac{1 \pm \sqrt{5}}{2}$ \\
$\chi_{3,+}$ & 3 & 3 & 3 & -1 & 0 & 0 & $\frac{1 \pm \sqrt{5}}{2}$ & $\frac{1 \pm \sqrt{5}}{2}$ \\
$\chi_{3,-}$ & 3 & 3 & 3 & -1 & 0 & 0 & $\frac{1 \mp \sqrt{5}}{2}$ & $\frac{1 \mp \sqrt{5}}{2}$ \\
$\chi_{4, \pm}$ & 4 & 4 & \pm 4 & 0 & 1 & \pm 1 & -1 & $\mp 1$ \\
$\chi_{5}$ & 5 & 5 & 5 & 1 & -1 & -1 & 0 & 0 \\
$\chi_{6}$ & 6 & 6 & -6 & 0 & 0 & 0 & 1 & -1 \\
$\chi_{7, k}$ & 120 & $\sigma_{j+k}(\alpha)$ & 0 & 0 & 0 & 0 & 0 & 0 \\
\hline
\end{tabular}

$j=1,2,3, \quad k=1,2,3, \quad \sigma_{n} \in \operatorname{Gal}\left(\mathbb{Q}\left(\zeta_{19}\right) / \mathbb{Q}\right), \quad \sigma_{n}\left(\zeta_{19}\right)=\zeta_{19}^{2^{n}}$,

$\alpha=3 \zeta_{19}-2 \zeta_{19}^{4}-2 \zeta_{19}^{6}+3 \zeta_{19}^{7}+3 \zeta_{19}^{8}-2 \zeta_{19}^{9}-2 \zeta_{19}^{10}+3 \zeta_{19}^{11}+3 \zeta_{19}^{12}-2 \zeta_{19}^{13}-2 \zeta_{19}^{15}+3 \zeta_{19}^{18}$

- $C_{23}^{2} \rtimes\left(C_{11} \times \mathrm{SL}(2,3)\right)$

\begin{tabular}{lrrrrcccccccc}
\hline & 1 & $23_{1}$ & $23_{2}$ & 2 & 4 & $3_{i}$ & $6_{i}$ & $11_{j}$ & $22_{j}$ & $44_{j}$ & $33_{i, j}$ & $66_{i, j}$ \\
\hline$\chi_{1, k}$ & 1 & 1 & 1 & 1 & 1 & $\zeta_{3}^{i k}$ & $\zeta_{3}^{i k}$ & $\zeta_{11}^{j k}$ & $\zeta_{11}^{j k}$ & $\zeta_{11}^{j k}$ & $\left(\zeta_{3}^{i} \zeta_{11}^{j}\right)^{k}$ & $\left(\zeta_{3}^{i} \zeta_{11}^{j}\right)^{k}$ \\
$\chi_{2, k}$ & 2 & 2 & 2 & -2 & 0 & $-\zeta_{3}^{i k}$ & $\zeta_{3}^{i k}$ & $2 \zeta_{11}^{j k}$ & $-2 \zeta_{11}^{j k}$ & 0 & $-\left(\zeta_{3}^{i} \zeta_{11}^{j}\right)^{k}$ & $\left(\zeta_{3}^{i} \zeta_{11}^{j}\right)^{k}$ \\
$\chi_{3, \ell}$ & 3 & 3 & 3 & 3 & -1 & 0 & 0 & $2 \zeta_{11}^{j \ell}$ & $3 \zeta_{11}^{j k}$ & $-\zeta_{11}^{j \ell}$ & 0 & 0 \\
$\chi_{264,1}$ & 264 & 11 & -12 & 0 & 0 & 0 & 0 & 0 & 0 & 0 & 0 & 0 \\
$\chi_{264,2}$ & 264 & -12 & 11 & 0 & 0 & 0 & 0 & 0 & 0 & 0 & 0 & 0 \\
\hline
\end{tabular}

$i=1,2, \quad j=1, \ldots, 10, \quad k=1, \ldots, 33, \quad \ell=1, \ldots, 10$ 
- $C_{59}^{2} \rtimes \mathrm{SL}(2,5)$

\begin{tabular}{rlrrrrrcc}
\hline & 1 & $59_{j}$ & 2 & 4 & 3 & 6 & $5_{ \pm}$ & $10_{ \pm}$ \\
\hline$\chi_{1}$ & 1 & 1 & 1 & 1 & 1 & 1 & 1 & 1 \\
$\chi_{2,+}$ & 2 & 2 & -2 & 0 & -1 & 1 & $\frac{-1 \pm \sqrt{5}}{2}$ & $\frac{1 \mp \sqrt{5}}{2}$ \\
$\chi_{2,-}$ & 2 & 2 & -2 & 0 & -1 & 1 & $\frac{-1 \mp \sqrt{5}}{2}$ & $\frac{1 \pm \sqrt{5}}{2}$ \\
$\chi_{3,+}$ & 3 & 3 & 3 & -1 & 0 & 0 & $\frac{1 \pm \sqrt{5}}{2}$ & $\frac{1 \pm \sqrt{5}}{2}$ \\
$\chi_{3,-}$ & 3 & 3 & 3 & -1 & 0 & 0 & $\frac{1 \mp \sqrt{5}}{2}$ & $\frac{1 \mp \sqrt{5}}{2}$ \\
$\chi_{4, \pm}$ & 4 & 4 & \pm 4 & 0 & 1 & \pm 1 & -1 & $\mp 1$ \\
$\chi_{5}$ & 5 & 5 & 5 & 1 & -1 & -1 & 0 & 0 \\
$\chi_{6}$ & 6 & 6 & -6 & 0 & 0 & 0 & 1 & -1 \\
$\chi_{7, k}$ & 120 & $\sigma_{j+k}(\alpha)$ & 0 & 0 & 0 & 0 & 0 & 0 \\
\hline & $=1, \ldots, 29$, & $k=1, \ldots, 29$, & $\sigma_{n} \in \mathrm{Gal}\left(\mathbb{Q}\left(\zeta_{59}\right) / \mathbb{Q}\right)$, & $\sigma_{n}\left(\zeta_{59}\right)=\zeta_{59}^{2 n}$, \\
$\alpha=$ & $\zeta_{59}-2 \zeta_{59}^{2}-\zeta_{59}^{3}+\zeta_{59}^{4}+\zeta_{59}^{5}-\zeta_{59}^{6}-\zeta_{59}^{7}-2 \zeta_{59}^{8}+3 \zeta_{59}^{10}+2 \zeta_{59}^{11}-2 \zeta_{59}^{12}+\zeta_{59}^{14}-\zeta_{59}^{16}-\zeta_{59}^{17}$ \\
& $+\zeta_{59}^{19}+2 \zeta_{59}^{21}-2 \zeta_{59}^{22}-\zeta_{59}^{23}+2 \zeta_{59}^{25}-2 \zeta_{59}^{26}+2 \zeta_{59}^{28}+\zeta_{59}^{29}+\zeta_{59}^{30}+2 \zeta_{59}^{31}-2 \zeta_{59}^{33}+2 \zeta_{59}^{34}$ \\
& $-\zeta_{59}^{36}-2 \zeta_{59}^{37}+2 \zeta_{59}^{38}+\zeta_{59}^{40}-\zeta_{59}^{42}-\zeta_{59}^{43}+\zeta_{59}^{45}-2 \zeta_{59}^{47}+2 \zeta_{59}^{48}+3 \zeta_{59}^{49}-2 \zeta_{59}^{51}-\zeta_{59}^{52}-\zeta_{59}^{53}$ \\
& $+\zeta_{59}^{54}+\zeta_{59}^{55}-\zeta_{59}^{56}-2 \zeta_{59}^{57}+\zeta_{59}^{58}$
\end{tabular}

\section{Further groups.}

- $C_{11}^{2} \rtimes Q_{12}$

\begin{tabular}{lrcccccc}
\hline & 1 & $11_{1, j}$ & $11_{2, j}$ & 2 & $4_{ \pm}$ & 3 & 6 \\
\hline$\chi_{1, k}$ & 1 & 1 & 1 & $(-1)^{k}$ & $( \pm i)^{k}$ & 1 & $(-1)^{k}$ \\
$\chi_{2, \pm}$ & 2 & 2 & 2 & \pm 2 & 0 & -1 & $\mp 1$ \\
$\chi_{3,1, \ell}$ & 12 & $\sigma_{j+\ell}(\alpha)$ & $\sigma_{j+\ell}(\beta)$ & 0 & 0 & 0 & 0 \\
$\chi_{3,2, \ell}$ & 12 & $\sigma_{j+\ell}(\beta)$ & $\sigma_{j+\ell}(\alpha)$ & 0 & 0 & 0 & 0 \\
\hline
\end{tabular}

$j=1, \ldots, 5, \quad k=1, \ldots, 4, \quad \ell=1, \ldots, 5, \quad \sigma_{n} \in \operatorname{Gal}\left(\mathbb{Q}\left(\zeta_{11}\right) / \mathbb{Q}\right), \quad \sigma_{n}\left(\zeta_{11}\right)=\zeta_{11}^{2^{n}}$

$\alpha=\zeta_{11}+\zeta_{11}^{2}+2 \zeta_{11}^{3}+2 \zeta_{11}^{4}+2 \zeta_{11}^{7}+2 \zeta_{11}^{8}+\zeta_{11}^{9}+\zeta_{11}^{10}$

$\beta=2+3 \zeta_{11}+\zeta_{11}^{4}+\zeta_{11}^{5}+\zeta_{11}^{6}+\zeta_{11}^{7}+3 \zeta_{11}^{10}$

- $C_{11}^{2} \rtimes\left(C_{5} \times Q_{12}\right)$

\begin{tabular}{|c|c|c|c|c|c|c|c|}
\hline & 1 & $11_{1}$ & $11_{2}$ & 2 & $4_{ \pm}$ & 3 & 6 \\
\hline$\chi_{1, k}$ & 1 & 1 & 1 & $(-1)^{k}$ & $( \pm i)^{k}$ & 1 & $(-1)^{k}$ \\
\hline$\chi_{2, \ell}$ & 2 & 2 & 2 & $2(-1)^{\ell}$ & 0 & -1 & $-1)^{\ell+1}$ \\
\hline$\chi_{3,1}$ & 60 & 5 & -6 & 0 & 0 & 0 & 0 \\
\hline$\chi_{3,2}$ & 60 & -6 & 5 & 0 & 0 & 0 & 0 \\
\hline & $5_{j}$ & & $10_{j}$ & $15_{j}$ & $20_{ \pm, j}$ & $30_{j}$ & \\
\hline$\chi_{1, k}$ & $\zeta_{5}^{j k}$ & & $\left.-\zeta_{5}^{j}\right)^{k}$ & $\zeta_{5}^{j k}$ & $\left( \pm i \zeta_{5}^{j}\right)^{k}$ & $\left(-\zeta_{5}^{j}\right)^{k}$ & \\
\hline$\chi_{2, \ell}$ & $2 \zeta_{5}^{j \ell}$ & & $\left.-\zeta_{5}^{j}\right)^{\ell}$ & $-\zeta_{5}^{j \ell}$ & 0 & $-\zeta_{5}^{j \ell}$ & \\
\hline$\chi_{3,1}$ & 0 & & 0 & 0 & 0 & 0 & \\
\hline$\chi_{3,2}$ & 0 & & 0 & 0 & 0 & 0 & \\
\hline
\end{tabular}


- $C_{7}^{2} \rtimes \mathrm{SL}(2,3) \quad(\operatorname{SmallGroup}(1176,214)$ in GAP $)$

\begin{tabular}{lrrcrcccc}
\hline & 1 & $7_{1}$ & $7_{2, i}$ & 2 & 4 & $3_{j}$ & $6_{j}$ & $21_{i, j}$ \\
\hline$\chi_{1, k}$ & 1 & 1 & 1 & 1 & 1 & $\zeta_{3}^{j k}$ & $\zeta_{3}^{j k}$ & $\zeta_{3}^{j k}$ \\
$\chi_{2, k}$ & 2 & 2 & 2 & -2 & 0 & $-\zeta_{3}^{j k}$ & $\zeta_{3}^{j k}$ & $-\zeta_{3}^{j k}$ \\
$\chi_{3}$ & 3 & 3 & 3 & 3 & -1 & 0 & 0 & 0 \\
$\chi_{4, k, \ell}$ & 8 & 1 & $\sigma_{i+\ell}(\alpha)$ & 0 & 0 & $2 \zeta_{3}^{j k}$ & 0 & $\left(\zeta_{7}^{2^{i+\ell}}+\zeta_{7}^{6 \cdot 2^{i+\ell}}\right) \cdot \zeta_{3}^{j k}$ \\
$\chi_{5}$ & 24 & -4 & 3 & 0 & 0 & 0 & 0 & 0 \\
\hline
\end{tabular}

$i=1,2,3, \quad j=1,2, \quad k=1,2,3, \quad \ell=1,2,3$,

$\sigma_{n} \in \operatorname{Gal}\left(\mathbb{Q}\left(\zeta_{7}\right) / \mathbb{Q}\right), \quad \sigma_{n}\left(\zeta_{7}\right)=\zeta_{7}^{2 n}, \quad \alpha=\zeta_{7}+3 \zeta_{7}^{3}+3 \zeta_{7}^{4}+\zeta_{7}^{6}$

- $C_{7}^{2} \rtimes\left(C_{9} \rtimes C_{4}\right) \quad(\operatorname{SmallGroup}(1764,64)$ in GAP $)$

\begin{tabular}{lccccccccccc}
\hline & 1 & $7_{1}$ & $7_{2, j}$ & 2 & $4_{ \pm}$ & 3 & $9_{k}$ & 6 & $18_{k}$ & $21_{ \pm}$ & $21_{j, \ell}$ \\
\hline$\chi_{1, r}$ & 1 & 1 & 1 & $(-1)^{r}$ & $( \pm i)^{r}$ & 1 & 1 & $(-1)^{r}$ & $(-1)^{r}$ & 1 & 1 \\
$\chi_{2, \pm}$ & 2 & 2 & 2 & \pm 2 & 0 & 2 & -1 & \pm 2 & $\mp 1$ & 2 & 2 \\
$\chi_{2, s, \pm}$ & 2 & 2 & 2 & \pm 2 & 0 & -1 & $\varrho_{k+s}(\alpha)$ & $\mp 1$ & $\pm \varrho_{k+s}(\alpha)$ & -1 & -1 \\
$\chi_{3}$ & 12 & 5 & -2 & 0 & 0 & 12 & 0 & 0 & 0 & 5 & -2 \\
$\chi_{4,+}$ & 12 & 5 & -2 & 0 & 0 & -6 & 0 & 0 & 0 & $\frac{-5 \pm 7 \sqrt{-3}}{2}$ & 1 \\
$\chi_{4,-}$ & 12 & 5 & -2 & 0 & 0 & -6 & 0 & 0 & 0 & $\frac{-5 \mp 7 \sqrt{-3}}{2}$ & 1 \\
$\chi_{5, t}$ & 12 & -2 & $\sigma_{j+t}(\beta)$ & 0 & 0 & 12 & 0 & 0 & 0 & -2 & $\sigma_{j+t}(\beta)$ \\
$\chi_{6, u}$ & 12 & -2 & $\sigma_{j+u}(\beta)$ & 0 & 0 & -6 & 0 & 0 & 0 & 1 & $\tau_{3 \ell+j+u}(\gamma)$ \\
\hline
\end{tabular}

$j=1,2,3 \quad k=1,2,3, \quad \ell=1,2, \quad r=1, \ldots, 4, \quad s=1,2,3, \quad t=1,2,3, \quad u=$ $1, \ldots, 6$,

$\varrho_{n} \in \operatorname{Gal}\left(\mathbb{Q}\left(\zeta_{9}\right) / \mathbb{Q}\right), \quad \varrho_{n}\left(\zeta_{9}\right)=\zeta_{9}^{2^{n}}, \quad \alpha=\zeta_{9}+\zeta_{9}^{-1}$,

$\sigma_{n} \in \operatorname{Gal}\left(\mathbb{Q}\left(\zeta_{7}\right) / \mathbb{Q}\right), \quad \sigma_{n}\left(\zeta_{7}\right)=\zeta_{7}^{2 n}, \quad \beta=\zeta_{7}-2 \zeta_{7}^{2}-2 \zeta_{7}^{5}+\zeta_{7}^{6}$,

$\tau_{n} \in \operatorname{Gal}\left(\mathbb{Q}\left(\zeta_{21}\right) / \mathbb{Q}\right), \quad \tau_{n}\left(\zeta_{21}\right)=\zeta_{21}^{2^{n}}, \quad \gamma=2 \zeta_{21}^{2}+2 \zeta_{21}^{4}+2 \zeta_{21}^{5}+2 \zeta_{21}^{7}+2 \zeta_{21}^{10}+\zeta_{21}^{11}+\zeta_{21}^{17}$

- $\left(C_{2}^{2} \times C_{7}\right) \rtimes C_{3} \quad\left(C_{3}\right.$ acts nontrivially on both $C_{2}^{2}$ and $\left.C_{7}\right)$

\begin{tabular}{lcrcccc}
\hline & 1 & 2 & $3_{i}$ & $7_{ \pm}$ & $14_{+, j}$ & $14_{-, j}$ \\
\hline$\chi_{1, k}$ & 1 & 1 & $\zeta_{3}^{i k}$ & 1 & 1 & 1 \\
$\chi_{2}$ & 3 & -1 & 0 & 3 & -1 & -1 \\
$\chi_{3,+}$ & 3 & 3 & 0 & $\frac{-1 \pm \sqrt{-7}}{2}$ & $\zeta_{7}+\zeta_{7}^{2}+\zeta_{7}^{4}$ & $\zeta_{7}^{3}+\zeta_{7}^{5}+\zeta_{7}^{6}$ \\
$\chi_{3,-}$ & 3 & 3 & 0 & $\frac{-1 \mp \sqrt{-7}}{2}$ & $\zeta_{7}^{3}+\zeta_{7}^{5}+\zeta_{7}^{6}$ & $\zeta_{7}+\zeta_{7}^{2}+\zeta_{7}^{4}$ \\
$\chi_{4, \ell,+}$ & 3 & -1 & 0 & $\frac{-1 \pm \sqrt{-7}}{2}$ & $\zeta_{7}^{3 \cdot 2^{j+\ell}}-\zeta_{7}^{5 \cdot 2^{j+\ell}}-\zeta_{7}^{6 \cdot 2^{j+\ell}}$ & $\zeta_{7}^{2^{j+\ell}}-\zeta_{7}^{2 \cdot 2^{j+\ell}}-\zeta_{7}^{4 \cdot 2^{j+\ell}}$ \\
$\chi_{4, \ell,-}$ & 3 & -1 & 0 & $\frac{-1 \mp \sqrt{-7}}{2}$ & $\zeta_{7}^{2^{j+\ell}}-\zeta_{7}^{2 \cdot 2^{j+\ell}}-\zeta_{7}^{4 \cdot 2^{j+\ell}}$ & $\zeta_{7}^{3 \cdot 2^{j+\ell}}-\zeta_{7}^{5 \cdot 2^{j+\ell}}-\zeta_{7}^{6 \cdot 2^{j+\ell}}$ \\
\hline
\end{tabular}

$i=1,2, \quad j=1,2,3, \quad k=1,2,3, \quad \ell=1,2,3$ 
- $\left(C_{3}^{3} \times C_{5}\right) \rtimes C_{52} \quad\left(C_{52}\right.$ acts transitively on both $C_{3}^{3}$ and $\left.C_{5}\right)$

\begin{tabular}{lrrrcccccccr}
\hline & 1 & 3 & 5 & $15_{ \pm}$ & 2 & $4_{ \pm}$ & 6 & $13_{j}$ & $26_{j}$ & $52_{j, \pm}$ & $65_{j}$ \\
\hline$\chi_{1, k}$ & 1 & 1 & 1 & 1 & $(-1)^{k}$ & $( \pm i)^{k}$ & 1 & $\zeta_{13}^{j k}$ & $\left(-\zeta_{13}^{j}\right)^{k}$ & $\left( \pm i \zeta_{13}^{j}\right)^{k}$ & $\zeta_{13}^{j k}$ \\
$\chi_{2, \ell}$ & 4 & 4 & -1 & -1 & 0 & 0 & 0 & $4 \zeta_{13}^{j \ell}$ & 0 & 0 & $-\zeta_{13}^{j \ell}$ \\
$\chi_{3, \pm}$ & 26 & -1 & 26 & -1 & \pm 26 & 0 & $\mp 1$ & 0 & 0 & 0 & 0 \\
$\chi_{4,+}$ & 52 & -2 & -13 & $\frac{1 \pm 3 \sqrt{-15}}{2}$ & 0 & 0 & 0 & 0 & 0 & 0 & 0 \\
$\chi_{4,-}$ & 52 & -2 & -13 & $\frac{1 \mp 3 \sqrt{-15}}{2}$ & 0 & 0 & 0 & 0 & 0 & 0 & 0 \\
\hline
\end{tabular}

$j=1, \ldots, 12, \quad k=1, \ldots, 52, \quad \ell=1, \ldots, 13$

- $\left(Q_{8} \times C_{7}\right) \rtimes C_{3} \quad\left(C_{3}\right.$ acts nontrivially on both $Q_{8}$ and $\left.C_{7}\right)$

\begin{tabular}{lrrrrccc}
\hline & 1 & 2 & $3_{i}$ & 4 & $6_{i}$ & $7_{ \pm}$ & $14_{ \pm}$ \\
\hline$\chi_{1, k}$ & 1 & 1 & $\zeta_{3}^{i k}$ & 1 & $\zeta_{3}^{i k}$ & 1 & 1 \\
$\chi_{2, k}$ & 2 & -2 & $-\zeta_{3}^{i k}$ & 0 & $\zeta_{3}^{i k}$ & 2 & -2 \\
$\chi_{3}$ & 3 & 3 & 0 & -1 & 0 & 3 & 3 \\
$\chi_{4,+}$ & 3 & 3 & 0 & 3 & 0 & $\frac{-1 \pm \sqrt{-7}}{2}$ & $\frac{-1 \pm \sqrt{-7}}{2}$ \\
$\chi_{4,-}$ & 3 & 3 & 0 & 3 & 0 & $\frac{-1 \mp \sqrt{-7}}{2}$ & $\frac{-1 \pm \sqrt{-7}}{2}$ \\
$\chi_{5, \ell,+}$ & 3 & 3 & 0 & -1 & 0 & $\frac{-1 \pm \sqrt{-7}}{2}$ & $\frac{-1 \pm \sqrt{-7}}{2}$ \\
$\chi_{5, \ell,-}$ & 3 & 3 & 0 & -1 & 0 & $\frac{-1 \mp \sqrt{-7}}{2}$ & $\frac{-1 \mp \sqrt{-7}}{2}$ \\
$\chi_{6,+}$ & 6 & -6 & 0 & 0 & 0 & $-1 \pm \sqrt{-7}$ & $1 \mp \sqrt{-7}$ \\
\hline
\end{tabular}

\begin{tabular}{lcc}
\hline & $28_{+, j}$ & $28_{-, j}$ \\
\hline$\chi_{1, k}$ & 1 & 1 \\
$\chi_{2, k}$ & 0 & 0 \\
$\chi_{3}$ & -1 & -1 \\
$\chi_{4,+}$ & $\zeta_{7}+\zeta_{7}^{2}+\zeta_{7}^{4}$ & $\zeta_{7}^{3}+\zeta_{7}^{5}+\zeta_{7}^{6}$ \\
$\chi_{4,-}$ & $\zeta_{7}^{3}+\zeta_{7}^{5}+\zeta_{7}^{6}$ & $\zeta_{7}+\zeta_{7}^{2}+\zeta_{7}^{4}$ \\
$\chi_{5, \ell,+}$ & $\zeta_{7}^{2^{j+\ell}}-\zeta_{7}^{2 \cdot 2^{j+\ell}}-\zeta_{7}^{4 \cdot 2^{j+\ell}}$ & $\zeta_{7}^{3 \cdot 2^{j+\ell}}-\zeta_{7}^{5 \cdot 2^{j+\ell}}-\zeta_{7}^{6 \cdot 2^{j+\ell}}$ \\
$\chi_{5, \ell,-}$ & $\zeta_{7}^{3 \cdot 2^{j+\ell}}-\zeta_{7}^{5 \cdot 2^{j+\ell}}-\zeta_{7}^{6 \cdot 2^{j+\ell}}$ & $\zeta_{7}^{j^{j+\ell}}-\zeta_{7}^{2 \cdot 2^{j+\ell}}-\zeta_{7}^{4 \cdot 2^{j+\ell}}$ \\
$\chi_{6,+}$ & 0 & 0 \\
\hline
\end{tabular}

$i=1,2, \quad j=1,2,3, \quad k=1,2,3, \quad \ell=1,2,3$

\section{References}

[1] S. D. Berman, On the theory of representations of finite groups, Doklady Akad. Nauk SSSR (N.S.), 86 (1952), 885-888.

[2] S. D. Berman, Characters of linear representations of finite groups over an arbitrary field, Mat. Sb. N.S., 44(86) (1958), 409-456.

[3] S. D. Berman and P. M. Gudivok, Indecomposable representations of finite groups over the ring of p-adic integers, Izv. Akad. Nauk SSSR Ser. Mat., 28 (1964), 875-910. 
[4] R. Brauer, A characterization of the characters of groups of finite order, Ann. of Math., 57 (1953), 357-377.

[5] J. Coates, p-adic L-functions and Iwasawa's theory, Algebraic number fields: $L$-functions and Galois properties (Proc. Sympos., Univ. Durham, 1975) Academic Press, London, 1977, 269-353.

[6] H. Cohen, Number Theory I, Tools and Diophantine Equations, Graduate Texts in Mathematics, 239, Springer, New York, 2007.

[7] H. Cohen, Number Theory II, Analytic and Modern Tools, Graduate Texts in Mathematics, 240, Springer, New York, 2007.

[8] J. H. Conway, R. T. Curtis, S. P. Norton, R. A. Parker and R. A. Wilson, Atlas of Finite Groups, Oxford University Press, Eynsham, 1985.

[9] B. N. Cooperstein, Maximal subgroups of $G_{2}\left(2^{n}\right)$, J. Algebra, 70(1) (1981), 23-36.

[10] M. Costantini and E. Jabara, On finite groups in which cyclic subgroups of the same order are conjugate, Comm. Algebra, 37(11) (2009), 3966-3990.

[11] D. A. Craven, The Theory of Fusion Systems: an algebraic approach, Cambridge Studies in Advanced Mathematics, 131, Cambridge University Press, Cambridge, 2011.

[12] C. W. Curtis and I. Reiner, Methods of Representation Theory. Vol. I, A Wiley-Interscience Publication, John Wiley \& Sons, Inc., New York, 1981.

[13] D. I. Deriziotis and G. O. Michler, Character table and blocks of finite simple triality groups ${ }^{3} D_{4}(q)$, Trans. Amer. Math. Soc., 303(1) (1987), 39-70.

[14] L. Dornhoff, The rank of primitive solvable permutation groups, Math. Z., 109 (1969), 205-210.

[15] L. Dornhoff, Group Representation Theory. Part A: Ordinary Representation Theory, Pure and Applied Mathematics, 7, Marcel Dekker, Inc., New York, 1971.

[16] J. A. Drozd and A. V. Roìter, Commutative rings with a finite number of indecomposable integral representations, Izv. Akad. Nauk SSSR Ser. Mat., 31 (1967), 783-798.

[17] R. H. Dye, On the involution classes of the linear groups $\mathrm{GL}_{n}(K), \mathrm{SL}_{n}(K)$, $\mathrm{PGL}_{n}(K), \mathrm{PSL}_{n}(K)$ over fields of characteristic two, Proc. Cambridge Philos. Soc., 72 (1972), 1-6.

[18] R. H. Dye, On the conjugacy classes of involutions of the unitary groups $\mathrm{U}_{m}(K), \mathrm{SU}_{m}(K), \mathrm{PU}_{m}(K), \mathrm{PSU}_{m}(K)$, over perfect fields of characteristic 2, J. Algebra, 24 (1973), 453-459.

[19] D. A. Foulser, Solvable primitive permutation groups of low rank, Trans. Amer. Math. Soc., 143 (1969), 1-54. 
[20] T. Fritzsche, The Brauer group of character rings, J. Algebra, 361 (2012), $37-40$.

[21] T. Fritzsche, Der Darstellungstyp des Charakterrings Einer Endlichen Gruppe, Friedrich Schiller University Jena, 2014.

[22] E. L. Green and I. Reiner, Integral representations and diagrams, Michigan Math. J., 25(1) (1978), 53-84.

[23] A. Heller and I. Reiner, Representations of cyclic groups in rings of integers, I, Ann. of Math., 76 (1962), 73-92.

[24] A. Heller and I. Reiner, Representations of cyclic groups in rings of integers, II, Ann. of Math., 77 (1963), 318-328.

[25] C. Hering, Transitive linear groups and linear groups which contain irreducible subgroups of prime order, Geometriae Dedicata, 2 (1974), 425-460.

[26] G. Higman, Suzuki 2-groups, Illinois J. Math., 7 (1963), 79-96.

[27] B. Huppert and N. Blackburn, Finite Groups II, Grundlehren der Mathematischen Wissenschaften 242, Springer-Verlag, Berlin-New York, 1982.

[28] B. Huppert and N. Blackburn, Finite Groups III, Grundlehren der Mathematischen Wissenschaften 243, Springer-Verlag, Berlin-New York, 1982.

[29] I. M. Isaacs, Character Theory of Finite Groups, Dover Publications, Inc., New York, 1994.

[30] H. Jacobinski, Sur les ordres commutatifs avec un nombre fini de réseaux indécomposables, Acta Math., 118 (1967), 1-31.

[31] A. Jones, Groups with a finite number of indecomposable integral representations, Michigan Math. J., 10 (1963), 257-261.

[32] H. E. Jordan, Group-characters of various types of linear groups, Amer. J. Math., 29(4) (1907), 387-405.

[33] P. B. Kleidman, The maximal subgroups of the Chevalley groups $G_{2}(q)$ with $q$ odd, the Ree groups ${ }^{2} G_{2}(q)$, and their automorphism groups, J. Algebra, 117(1) (1988), 30-71.

[34] G. Navarro and J. Tent, Rationality and Sylow 2-subgroups, Proc. Edinb. Math. Soc., 53 (2010), 787-798.

[35] A. Raggi-Cárdenas, Burnside rings of finite representation type, Bull. Austral. Math. Soc., 42(2) (1990), 247-251.

[36] U. Reichenbach, Modultheorie von Burnsideringen Endlicher Gruppen, Mathematica Gottingensis 12, 1997.

[37] J.-P. Serre, Lineare Darstellungen Endlicher Gruppen, Akademie-Verlag, Berlin, 1972.

[38] E. E. Shult, On finite automorphic algebras, Illinois J. Math., 13 (1969), 625653. 
[39] W. A. Simpson and J. S. Frame, The character tables for $\mathrm{SL}(3, q)$, SU $\left(3, q^{2}\right)$, $\operatorname{PSL}(3, q), \operatorname{PSU}\left(3, q^{2}\right)$, Canad. J. Math., 25 (1973), 486-494.

[40] R. Steinberg, The representations of $\mathrm{GL}(3, q)$, $\mathrm{GL}(4, q), \operatorname{PGL}(3, q)$, and PGL $(4, q)$, Canad. J. Math., 3 (1951), 225-235.

[41] M. Suzuki, On a class of doubly transitive groups, Ann. of Math., 75 (1962), 105-145.

[42] H. N. Ward, On Ree's series of simple groups, Trans. Amer. Math. Soc., 121 (1966), 62-89.

[43] R. A. Wilson, The Finite Simple Groups, Graduate Texts in Mathematics, 251, Springer-Verlag London, Ltd., London, 2009.

[44] H. Yamaki, The order of a group of even order, Proc. Amer. Math. Soc., 136(2) (2008), 397-402.

\section{Tim Fritzsche}

Mathematical Institute

Friedrich Schiller University

07737 Jena, Germany

e-mail: Tim.Fritzsche@uni-jena.de 